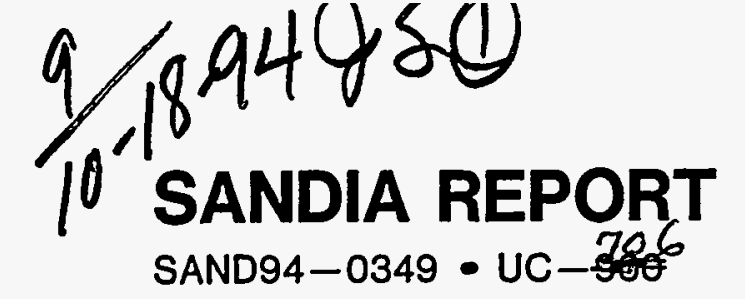

Unlimited Release

Printed September 1994

\title{
Detection Optimization Using Linear Systems Analysis of a Coded Aperture Laser Sensor System
}

S. M. Gentry

Propared by

Sandla Natlonal Laboratorles

Albuquerque, Now Mexlco 87185 and Livermore, Callfornla 94550

tor the United States Department of Energy

under Contract DE-AC04-94AL85000

Approved for public release; distribution is unlimited.

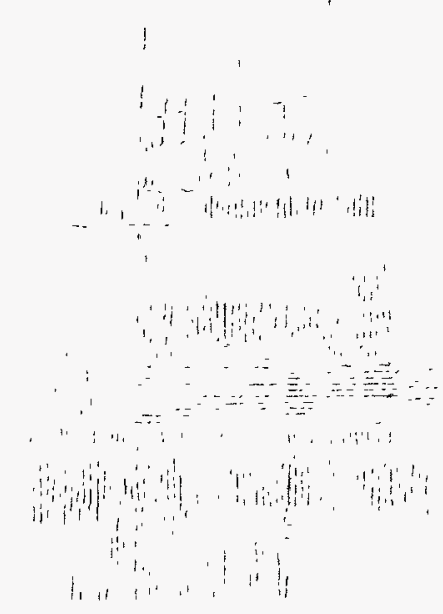


Issued by Sandia National Laboratories, operated for the United States Department of Energy by Sandia Corporation.

NOTICE: This report was prepared as an account of work sponsored by an agency of the United States Government. Neither the United States Government nor any agency thereof, nor any of their employees, nor any of their contractors, subcontractors, or their employees, makes any warranty, express or implied, or assumes any legal liability or responsibility for the accuracy, completeness, or usefulness of any information, apparatus, product, or process disclosed, or represents that its use would not infringe privately owned rights. Reference herein to any specific commercial product, process, or service by trade name, trademark, manufacturer, or otherwise, does not necessarily constitute or imply its endorsement, recommendation, or favoring by the United States Government, any agency thereof or any of their contractors or subcontractors. The views and opinions expressed herein do not necessarily state or reflect those of the United States Government, any agency thereof or any of their contractors.

Printed in the United States of America. This report has been reproduced directly from the best available copy.

Available to DOE and DOE contractors from Office of Scientific and Technical Information PO Box 62

Oak Ridge, TN 37831

Prices available from (615) 576-8401, FTS 626-8401

Available to the public from

National Technical Information Service

US Department of Commerce

5285 Port Royal RD

Springfield, VA 22161

NTIS price codes

Printed copy: A04

Microfiche copy: A06 


\section{DISCLAIMER}

Portions of this document may be illegible in electronic image products. Images are produced from the best available original document. 
SAND94-0349

Distribution

Unlimited Release

Category UC- 706

Printed September, 1994

\title{
DETECTION OPTIMIZATION USING LINEAR SYSTEMS ANALYSIS OF A CODED APERTURE LASER SENSOR SYSTEM
}

\author{
Stephen M. Gentry \\ Optoelectronic Design Department \\ Sandia National Laboratories \\ Albuquerque, New Mexico 87185
}

\begin{abstract}
Minimum detectable irradiance levels for a diffraction grating based laser sensor were calculated to be governed by clutter noise resulting from reflected earth albedo. Features on the earth surface caused pseudo-imaging effects on the sensor's detector arrays that resulted in the limiting noise in the detection domain. It was theorized that a custom aperture transmission function existed that would optimize the detection of laser sources against this clutter background. Amplitude and phase aperture functions were investigated. Compared to the diffraction grating technique, a classical Young's double-slit aperture technique was investigated as a possible optimized solution but was not shown to produce a system that had better clutter-noise limited minimum detectable irradiance.

Even though the double-slit concept was not found to have a detection advantage over the slitgrating concept, one interesting concept grew out of the double-slit design that deserved mention is this report, namely the Barker-coded double-slit. This diffractive aperture design possessed properties that significantly improved the wavelength accuracy of the double-slit design.
\end{abstract}

While a concept was not found to beat the slit-grating concept, the methodology used for the analysis and optimization is an example of the application of optoelectronic system-level linear analysis. The techniques outlined here can be used as a template for analysis of a wide range of optoelectronic systems where the entire system, both optical and electronic, contribute to the detection of complex spatial and temporal signals.

Funding for this project was provided under the Laboratory Directed Research and Development Program at Sandia National Laboratories for the United States Department of Energy. 


\section{ACKNOWLEDGMENTS}

The author wishes to recognize the input of Kurt Lanes for initial ideas concerning improving the performance of the slit-grating concept as well as guidance and reflection concerning implementation of the optimization. Kurt was also the source of the idea for using Barker codes to improve the double-slit design. Rex Kay provided additional help and guidance with ideas as they came up. Rex's comment "the stuff of life seems always to be conserved" (loosely paraphrased) came true in the case of this project. Mike Rogers developed the optimal filter used with the slitgrating concept as well as provided guidance to a novice in the development of the figure of merit definition. Acknowledgment also needs to go to the team responsible for developing the entire slitgrating concept who are too numerous to mention here. 


\section{TABLE OF CONTENTS}

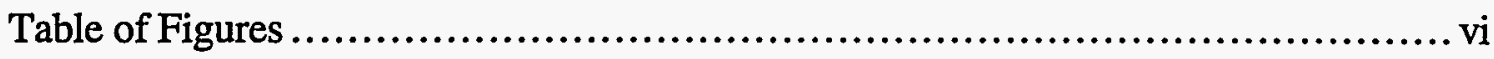



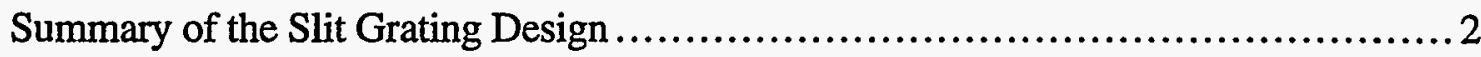

Linear Systems Approach for End-to-End Electro-Optical System Modeling............... 5

Frequency Domain Representations of Laser Signals and Clutter Noise in a Simple Pupil/Lens Optical System ..............................................5

Fraunhoffer Diffraction from a General Pupil Function ..........6 6

Fresnel Diffraction after a Lens from a General Pupil Function . . 8

Monochromatic Diffraction PSF......................................9

Calculation of the Laser Signal Spatial Frequency Spectrum ..... 10

Effects of the Optical Aberrations on the Signal Irradiance

Distribution in the Focal Plane................................... 11

Calculation of Clutter Noise Spatial Frequency Spectrum.................... 11

Polychromatic Diffraction PSF .............................. 12

Scene Definition and Clutter Noise Representation Using the Diffraction and Geometric PSF's............................ 12

Frequency Domain Representation a Detector Matrix Array .................... 13

Simple Analog Filter Transfer Function ...................................... 18

Simple Digital Filtering Transfer Function .................................. 18

Signal-to-Noise Ratio Analysis for Laser Detection Against Clutter Noise ....... 19

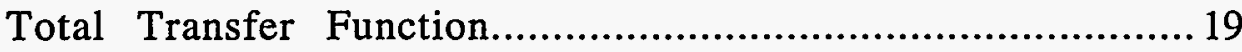

RMS Signal-to-Noise Ratio Definition ............................... 19

System Response to Laser Signal ................................. 20

System Response to Clutter Noise .................................. 20

Net Figure-of-Merit Definition for Clutter-Limited SNR...................... 21

Application of Linear System Analysis to the Slit-Grating Concept for an Estimate of

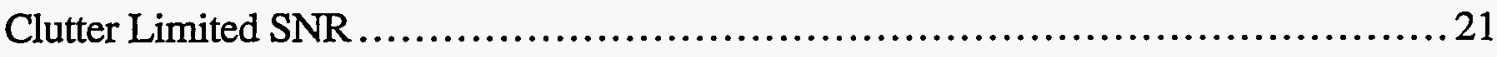

Toric Lens Equivalent Cylindrical System ................................21 
Slit-Grating Pupil Function ............................................ 22

Slit-Grating Monochromatic Diffraction PSF and Signal Spectrum................ 22

Geometric PSF .......................................................... 24

Linear Detector Array ................................................. 24

Analog and Digital Filtering.............................................. 25

Computation of Net Signal Amplitude Spectrum.............................26

Computation of Net Clutter Noise Amplitude Spectrum...............................27

Polychromatic Diffraction PSF for the Slit-Grating Concept .............22

Scene Data Used as a Source for Clutter Noise...............................22

Computation of a for the Slit-Grating Design .............................. 29

Analysis of Slit-Grating Concept for Determining Optimum Pupil Function ............ 29

Brief Discussion of Phase-Only Pupil Functions as Replacements for the Slit-Grating

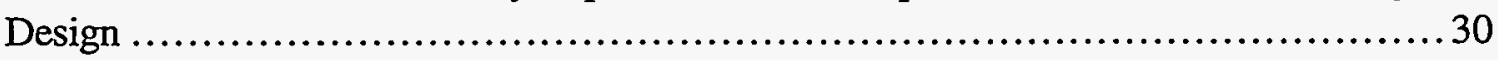

Application of Linear System Analysis to Young's Double Slit: A Proposed Optimum

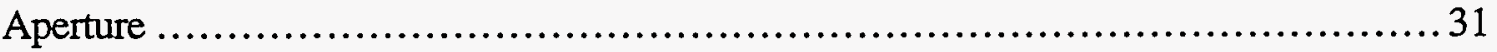

Comparison of a Between the Double-Slit and Slit-Grating Designs .................. 38

A Brief Discussion of the Barker Coded Double-Slit Concept..................................38

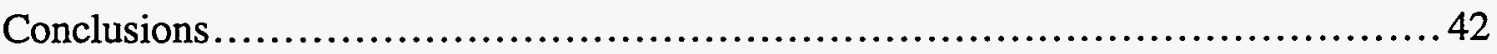

Appendix A: Barker Coded Double Slit Design Results............................ 43 


\section{TABLE OF FIGURES}

Figure 1: Slit Grating Concept..............................................................

Figure 2: Ronchi Ruling Grating Amplitude Transmission Function .......................... 3

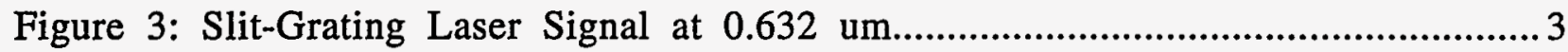

Figure 4: Slit Grating Normalized Laser Signal Power Spectrum at 0.632 um.................. 4

Figure 5: Theoretical Clutter Background Signal Resulting From Background Scene.................. 4

Figure 6: Normalized Clutter Background Signal Power Spectrum..................................... 4

Figure 7: Fraunhoffer Diffraction ............................................................ 8

Figure 8: Fresnel Diffraction Geometry with Aperture at the Lens...........................9

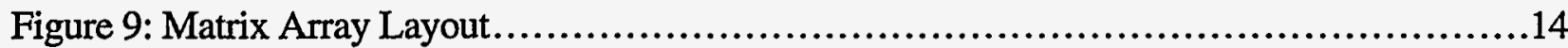

Figure 10: Aliasing in Sampled Systems ................................................

Figure 11: Cylindrical Equivalent to the Toric Lens...................................................22

Figure 12: Slit Grating Monochromatic Diffraction PSF at $\lambda=1.06 \mu \mathrm{m} \ldots \ldots \ldots \ldots \ldots \ldots \ldots \ldots . .23$

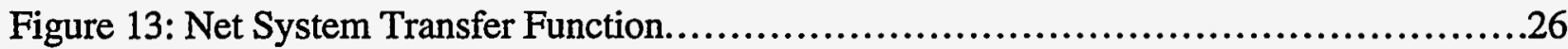

Figure 14: Laser Signal Amplitude Spectrum at $1.06 \mu \mathrm{m}$ Before and After System Transfer

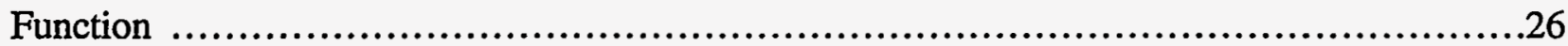

Figure 15: Slit-Grating Polychromatic Diffraction PSF ...................................27

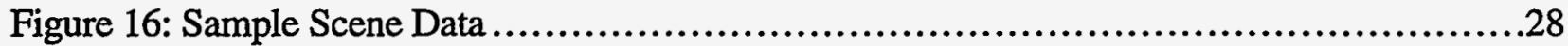

Figure 17: Residual RMS Clutter Noise Results ...........................................29

Figure 18: Variation of $\alpha$ with Wavelength for the Slit-Grating Design .......................30

Figure 19: Laser Signal Frequency Spectra for 60 um Slit Spacing......................................34

Figure 20: Laser Signal Frequency Spectra for 50 um Slit Spacing..................................35

Figure 21: Sample-Space Laser Signal Profiles vs. Wavelength for 50 um Slit Spacing.............36

Figure 22: Polychromatic Diffraction PSF for the 50 um Double-Slit Design .....................37

Figure 23: Clutter Noise Spectrum for the 50 um Double-Slit Design .........................38

Figure 24: Variation of $\alpha$ with Wavelength for the Double-Slit Design ........................39

Figure 25: SNR Improvement of the Double-Slit Design Over the Slit-Grating Design............39

Figure 26: Peak Signal vs. Barker Code Length ........................................41

Figure A1: Young's Double Slit (1 Bit Barker Code)....................................................43

Figure A2: 7 Bit Barker Code Results ................................................... 43

Figure A3: 8 Bit Barker Code Results ................................................44

Figure A4: 9 Bit Barker Code Results .................................................44

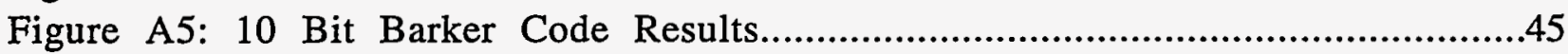

Figure A6: 11 Bit Barker Code Results...............................................................46

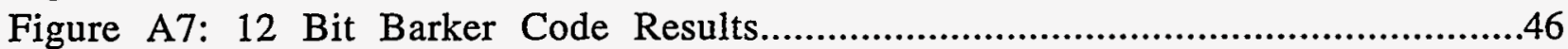

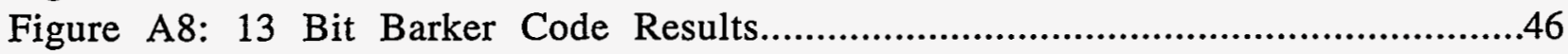

Figure A9: 14 Bit Barker Code Results..............................................................47

Figure A10: 15 Bit Barker Code Results ...............................................47

Figure A11: 16 Bit Barker Code Results ...............................................48

Figure A12: 17 Bit Barker Code Results .............................................48 
Figure A13: 18 Bit Barker Code Results .............................................49

Figure A14: 19 Bit Barker Code Results ...............................................49

Figure A15: 20 Bit Barker Code Results .............................................5 50

Figure A16: 21 Bit Barker Code Results ..............................................50

Figure A17: 22 Bit Barker Code Results ..............................................51

Figure A18: 23 Bit Barker Code Results ..............................................51

Figure A19: 24 Bit Barker Code Results ............................................5. 52

Figure A20: 25 Bit Barker Code Results .............................................52

Figure A21: 26 Bit Barker Code Results ..............................................53

Figure A22: 27 Bit Barker Code Results ............................................53

Figure A23: 28 Bit Barker Code Results .............................................54

Figure A24: 29 Bit Barker Code Results ..............................................54

Figure A25: 30 Bit Barker Code Results ...........................................55

Figure A26: 31 Bit Barker Code Results ............................................55

Figure A27: 32 Bit Barker Code Results .............................................56

Figure A28: 33 Bit Barker Code Results .............................................56 


\section{INTRODUCTION}

Linear systems theory is extensively used in the design of analog and digital electronic systems. Its usage allows an integrated approach to designing the time-dependent operation of a system as a whole. The designer is allowed to specify the operating characteristics of the components in a system in the frequency domain, where magnitude responses multiply and phase responses add. Similar analysis can be done in the time domain but require convolution operations which are not as intuitively obvious. These same linear systems techniques can be applied to optical systems, providing an intuitive technique for analyzing an entire system in the spatial frequency domain. Gaskill ${ }^{1}$ and Goodman ${ }^{2}$ discuss in detail these techniques in their application to optical systems. The following sections on optical linear systems theory are included to provide a common basis for the discussion of an overall design and optimization approach for improvement of this sensor system.

It is the intention of this document to apply these linear systems techniques in both the optical and electronic portions of a specific complex electro-optical system to optimize the relative RMS signalto-clutter-noise ratio. Signal-to-noise ratio for a standard signal and a standard background scene is chosen as a relative figure of merit by which the system is optimized because it simplifies the analysis to only those parameters that contribute to the SNR.

The specific system under investigation is a diffraction grating-based system, hereafter called the slit-grating concept, which is used for the detection of laser illumination. The minimum operational requirements of this system were that it be able to yield information concerning the wavelength, direction of arrival, and illumination level of a laser source aimed at the system. The goal of this research is to investigate changing the aperture function of this system in a way that optimizes detection of the laser source in the presence of background illumination. It was theorized that the selection of a diffraction grating as an aperture function may not have optimized detection and that some other, more general function could be synthesized that would optimize detection and still yield the same data products. Such a function would be allowed to have a general transmission function which could modulate the phase and/or amplitude of the signal and background optical wavefronts.

The problem was divided into two general classes, amplitude functions and phase functions, with the intent that lessons learned in each class could be applied to develop a general optimum transmission function that could include both phase and amplitude modulation. This general function is termed a coded aperture, in that the optimum transfer function would be coded into the transmission function of the aperture.

\footnotetext{
${ }^{1}$ Jack D. Gaskill, Linear System. Fourier Transforms and Optics (John Wiley and Sons, 1978)

2Joseph W. Goodman, Introduction to Fourier Optics (McGraw-Hill Physical and Quantum Electronics Series, McGraw-Hill Inc., 1968)
} 
The following discussion will first outline the general method of the application of linear systems theory to the slit-grating concept, with the goal of computing a figure-of-merit related to the clutternoise-limited SNR for the system. The results of this initial analysis will be analyzed to determine the upgrade path to the optimum aperture function that will yield maximum SNR and still perform the required operation and yield the required data products. Both amplitude and phase apertures will be discussed and the resulting Young's double slit approach will be examined as a possible optimum, but will be shown not to have significantly improved performance over the slit-grating concept.

Even though the double-slit design was not successful in improving the clutter-limited SNR, one novel design improvement deserves mention in this report. It was found that a Barker-coded double-slit could significantly improve the wavelength accuracy of a double-slit design without significantly decreasing the clutter-limited SNR.

\section{SUMMARY OF THE SLIT GRATING DESIGN}

The slit-grating concept shown in figure 1 includes a toroidal lens imaging onto a linear detector array. A narrow slit Ronchi-ruling diffraction grating shown in figure 2 defines the aperture of the system and causes the system point-spread function to be diffracted into a series of three spots on the array. The location of the zero-order spot gives information concerning the angle-of-arrival vector of the incident beam in the plane containing the array and the optical axis, and the locations of the first diffracted order spots, relative to the zero order spot, indicate the wavelength of the incident laser illumination. To form a complete direction-of-arrival solution, data is required from a minimum of two subassemblies rotated relative to each other by some appreciable angle.

This system was designed to operate while looking down at the earth in the presence of a reflected solar background. Because of the narrowband nature of their spectra relative to the broad solar spectra, laser sources appear as narrow spikes riding on top of a slowly varying background signal. This solar background signal is the limiting noise in the system. Examples of the signal and background are shown in figures 3-6 including their corresponding spatial frequency spectrums.

Both the signal and noise spatial frequency spectra are spread over the same spectral regions with severe overlap in the lower frequencies. If a diffracting aperture could be developed that seperated signal spatial frequency content from the background, filtering techniques could be adjusted to further enhance the signal and reject the noise. This is the hypothesis that will be investigated in the following sections.

Since the spatial frequency representation of the system seems to lead to the hypothesis stated above, it seems that a technique which allows one to analyze a system in the spatial-frequency domain would be advantageous in providing guidance in approach to system optimization. Linear systems analysis is such an approach. 


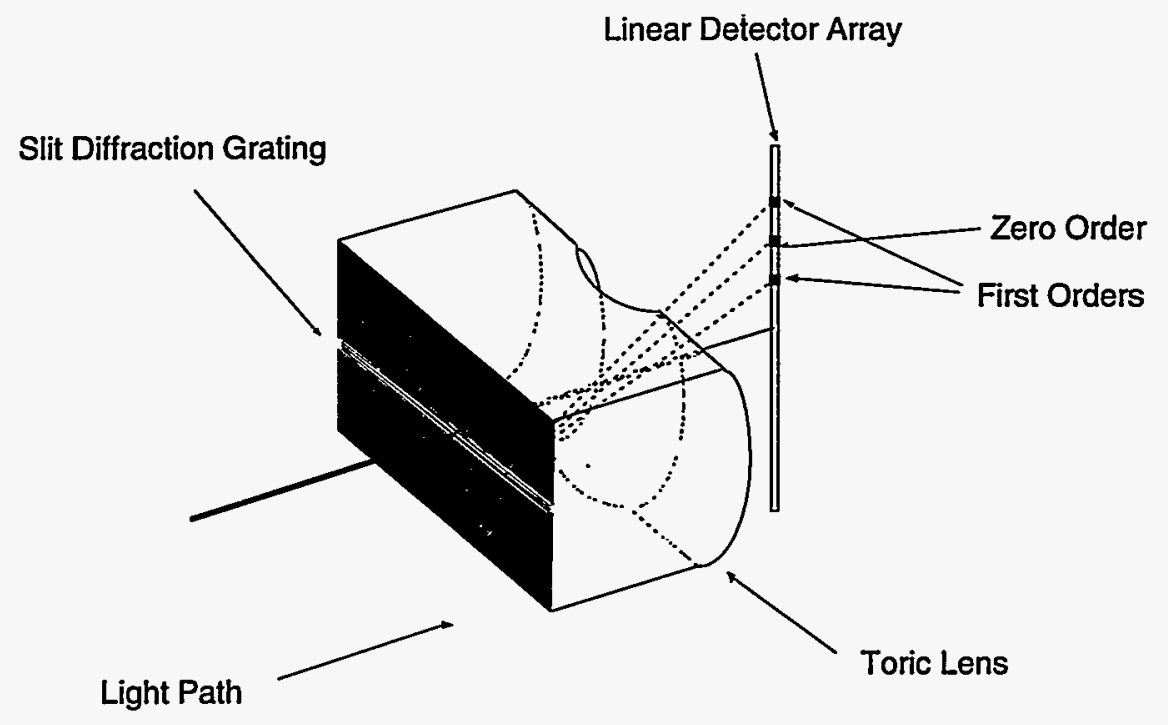

Figure 1: Slit Grating Concept

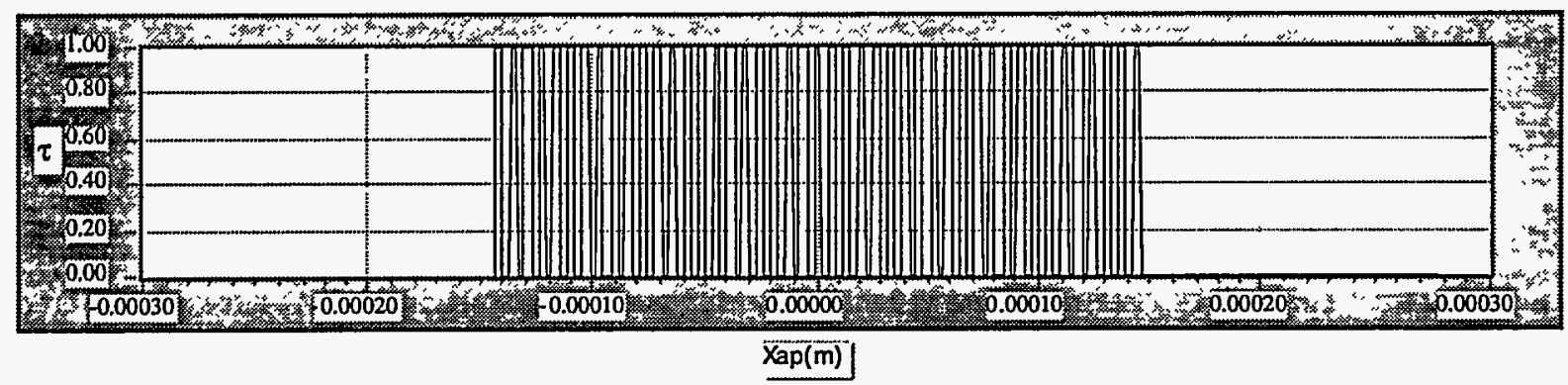

Figure 2: Ronchi Ruling Grating Amplitude Transmission Function

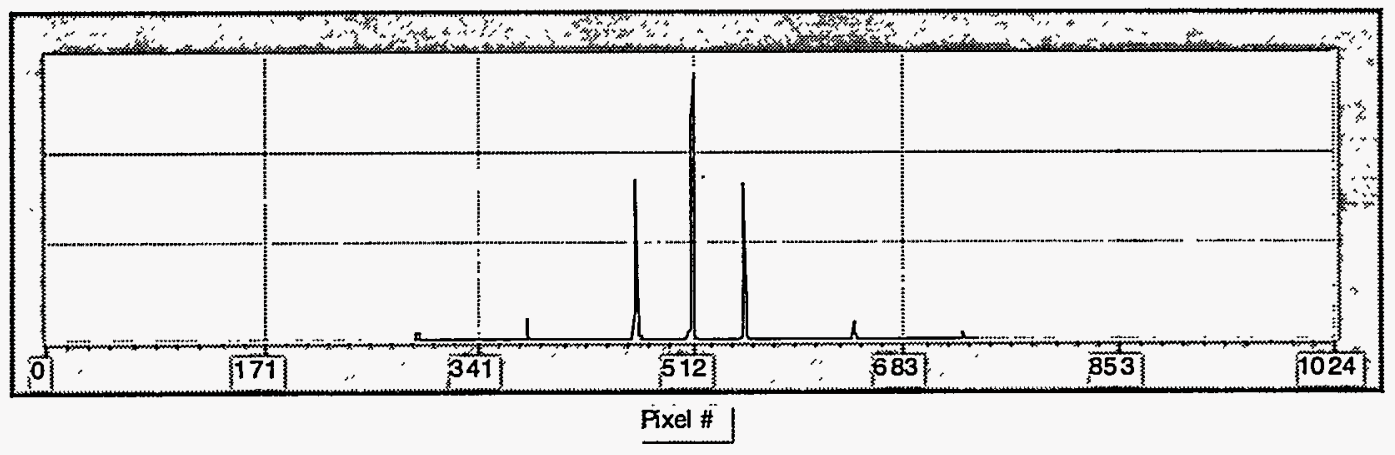

Figure 3: Slit-Grating Laser Signal at 0.632 um 


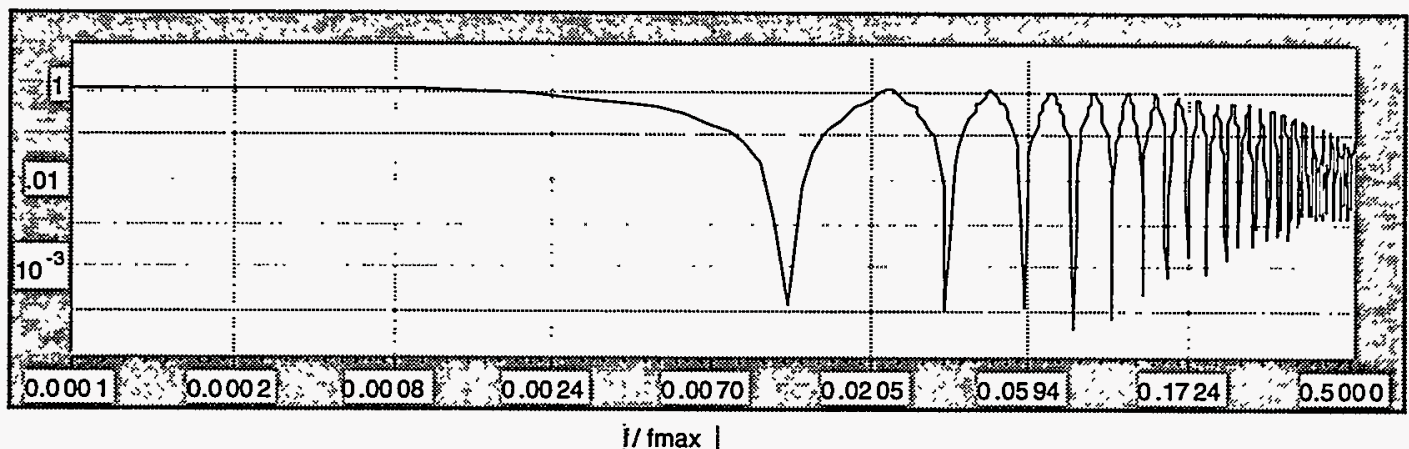

Figure 4: Slit Grating Normalized Laser Signal Power Spectrum at .632 um

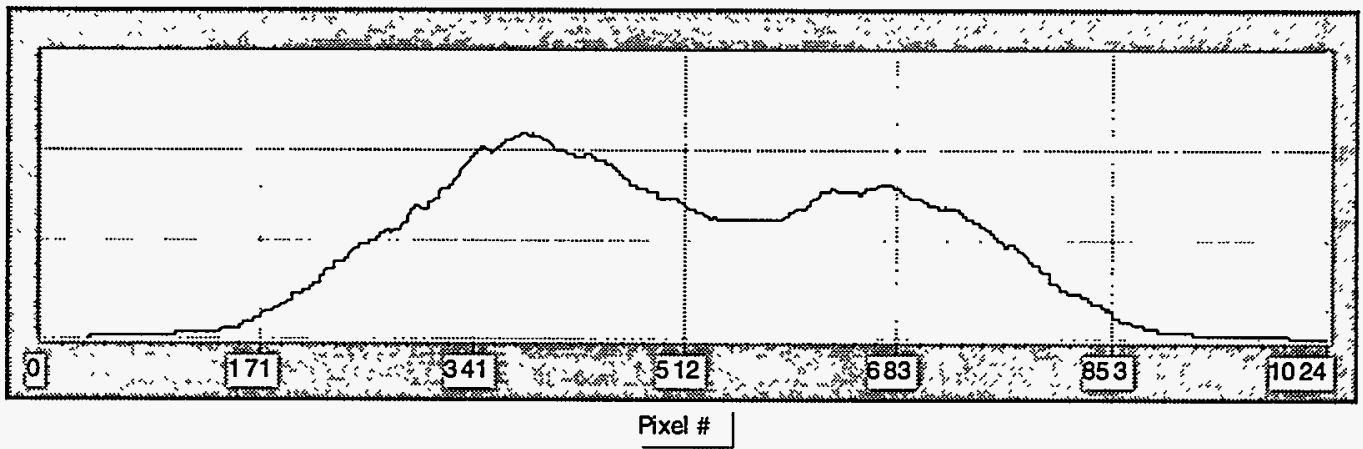

Figure 5: Theoretical Clutter Background Signal Resulting From Background Scene

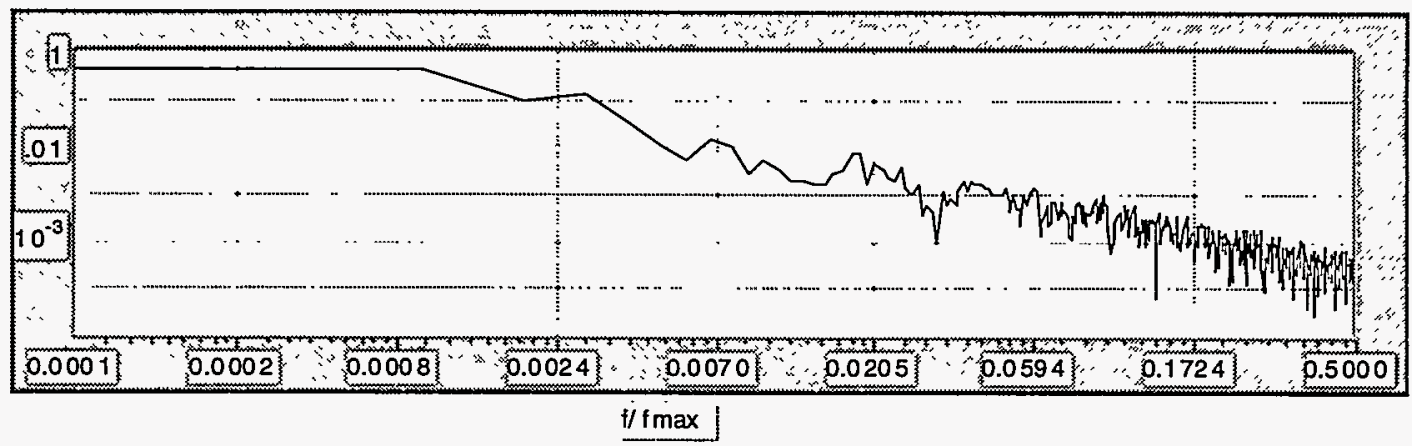

Figure 6: Normalized Clutter Background Signal Power Spectrum 


\section{LINEAR SYSTEMS APPROACH FOR END-TO-END ELECTRO-OPTICAL SYSTEM MODELING}

The advantage of a linear systems approach for end-to-end system modeling is that it allows the designer to examine the frequency dependent characteristics of each element in a system as it effects the whole. For example, the optical system has a spatial frequency response that it imparts to the scene that it is imaging. It presents a 'filtered' signal to the detector system intended to receive this image and convert it to an electrical signal. The detector system has both a spatial and temporal frequency response that it in turn imparts to the scene passed to it by the optical system. The analog and digital electronic subsystems have frequency responses that can be described using traditional linear electronic systems theory which further filter the signal read out of the detector array. As long as linear systems are used as components in each step of the model, the entire system can be described as cascading amplitude and phase transformation steps. This report will assume that all the filtering components have linear phase response and can be modeled only by their magnitude responses.

If this technique were used to rigorously model entire systems, it would allow the designer to accurately specify components that are appropriate for the signals that are expected at each point in the processing. For example, digital and analog filters are routinely designed for systems based on expected signal spectra. This design decision is usually made by an electronic engineer or software analyst without knowledge of the filtering that has already been performed on the signal by the optical system. Overall modulation transfer function budgets, typically found in optical design, can include degradation or enhancement due to analog or digital filtering techniques. As long as there is a unified approach to electro-optical system design, prudent choices at all levels of the system design can be made that keep in mind the effects of components on the entire system.

Following is a discussion of the three major elements of an optical system that will be used in the analysis of the slit-grating concept. These three components are the pupil function, the lens and the detector. With the spatial frequency representation of these elements and the frequency response of the electronic systems, the entire system can be modeled in the frequency domain.

With the component models expressed functionally in the frequency domain, the models will be combined to functionally describe the expected signal and background noise functions as they are seen through the entire system. A simple definition of a figure of merit based on RMS clutterlimited SNR is then able to be written which is then able to be used to compare system performance between system concepts.

\section{Frequency Domain Representations of Laser Signals and Clutter Noise in a Simple Pupil/Lens Optical System}

The following discussion of linear optical systems is discussed with formulations that include three-dimensional variation of the properties of an optical system. This is done to provide a complete discussion of the application of these techniques to optical systems in general which require the full three-dimensional application of the equations. It will be seen later in this report that 
all of the following three dimensional relationships can be made two-dimensional to represent analysis of the slit-grating concept.

The ultimate limitation of the image forming properties of an optical system lies in the diffracting nature of the aggregate pupil function of an entire optical system. Gaskill ${ }^{3}$ discusses the complex interaction of apertures, lenses and mirrors in an optical system which results in the diffraction point-spread-function (PSF) of the system. Gaskill's notation will be used throughout this discussion. The diffraction PSF of an optical system relates to the interaction of the optical wavefronts with the apertures in the optical system. Below is the general discussion of the development of the diffraction PSF for a system comprised of only a general transmitting aperture, which will then be extended to a system with both a lens and a general aperture function.

\section{Fraunhoffer Diffraction from a General Pupil Function}

Under the Fraunhoffer assumptions discussed below, the diffraction PSF of an optical system can be determined from a scaled two-dimensional Fourier transform of the pupil function of the system. For example, a system where there are no lenses or mirrors, only an aperture with a general aperture transmission function $t_{1}(x, y)$ which is illuminated by a wavefield $u_{1}^{-}(x, y)$, will cause a diffracted wave field of $u_{2}(x, y)$ at plane $z=z_{2}$ in the far-field, or 'Fraunhoffer', region that is given by the scaled two-dimensional Fourier transform of the wave function after passing through the aperture. The graphical layout of this case is depicted in figure 7.

The transmission function that represents the amplitude and phase transformation made on the wave as it passes through the pupil is shown below as a general complex function, $t_{1}(x, y)$ with amplitude and phase variation as a function of $\mathrm{x}$ and $\mathrm{y}$.

$$
\begin{gathered}
t_{1}(x, y)=\tau(x, y) e^{j k \phi(x, y)} \\
k=\frac{2 \pi}{\lambda}
\end{gathered}
$$

$$
\begin{aligned}
& \tau(x, y)=\text { aperture amplitude transmission variation } \\
& \phi(x, y)=\text { aperture phase delay variation (rad) } \\
& \lambda=\text { optical wavelength; }
\end{aligned}
$$

The illuminating wave, $u_{1}^{-}(x, y)$, will be considered a plane-wave arriving from a compound angle in the field.

$$
\begin{gathered}
u_{1}^{-}(x, y)=A e^{j\left[k\left(\gamma_{x} x+\gamma_{y} y\right)+\Phi\right]} \\
\gamma_{x, y}=\text { direction cosines of propagation vector in }(\mathrm{x}, \mathrm{z}) \text { plane }
\end{gathered}
$$

${ }^{3}$ Gaskill, ch. 10-11 


$$
\begin{aligned}
& \Phi=\text { initial phase of wavefront } \\
& \text { A = wave amplitude }
\end{aligned}
$$

The wave exiting the pupil, $u_{1}^{+}(x, y)$, is an amplitude and phase modulated version of the input wave based on the pupil function.

$$
u_{1}^{+}(x, y)=u_{1}^{-}(x, y) \cdot t_{1}(x, y)
$$

Gaskill ${ }^{4}$ proves that the resulting wavefield, after propagating a significant distance, can be represented as a scaled Fourier transform of the pupil function.

$$
u_{2}(x, y)=\left(\frac{1}{\lambda z_{12}}\right)\left\{F F\left[u_{1}^{+}(x, y)\right]\right\}_{\xi=x / \lambda z_{12}, \eta=y / \lambda z_{12}}
$$

$$
\begin{aligned}
& F F[\ldots]=\text { two dimensional Fourier transform } \\
& z_{12}=\text { propagation path from aperture and observation plane } \\
& \xi=\mathrm{x} \text {-direction spatial frequency variable } \\
& \eta=\text { y-direction spatial frequency variable }
\end{aligned}
$$

The irradiance that one would observe at plane $\mathrm{z}=\mathrm{z}_{2}$ is proportional to the squared-modulus of the wave field.

$$
\dot{L}_{2}(x, y)=\left|u_{2}(x, y)\right|^{2}=\left(\frac{1}{\lambda z_{12}}\right)^{2}\left|F F\left[u_{1}^{+}(x, y)\right]\right|_{\xi=x / \lambda z_{12}, \eta=y / \lambda z_{12}}^{2}
$$

The Fraunhoffer, or far-field, assumption made for the above formulations to be valid is that the wave must propagate a significant distance with respect to the size of the aperture.

$$
\left|z_{12}\right| \gg>\frac{\pi L_{1}^{2}}{\lambda}
$$

$$
L_{1}=\text { maximum extent of the diffracting aperture }
$$

A similar constraint is placed on the maximum radial extent relative to the axis of the system that the formulations are valid.

$$
L_{2}^{4} \ll \frac{4 \lambda\left|z_{12}\right|^{2}}{\pi}
$$




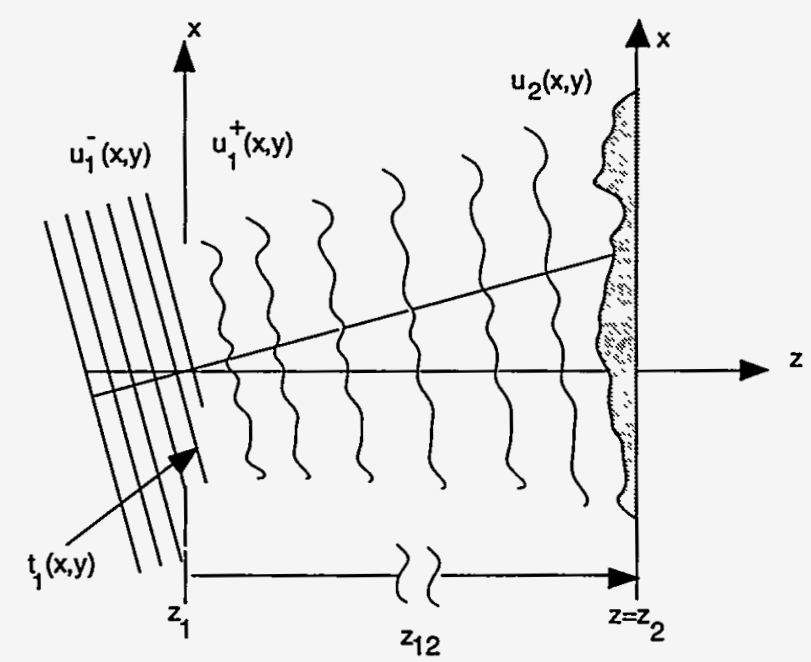

Figure 7: Fraunhoffer Diffraction

Fresnel Diffraction after a Lens from a General Pupil Function

Gaskill 5 indicates that a similar Fourier-transform relationship is true in the configuration where there is a lens following the diffracting aperture which focuses the wavefronts onto a focal plane. In this case of Fresnel, or near-field diffraction, the Fraunhoffer 'region' is collapsed onto the focal plane of the lens system. Thus the form of the diffraction pattern seen in the far-field is identical to the near-field pattern when focused by a lens. The only differences being in linear scale and peak amplitude. If the incident illumination is a plane wave with only linear-phase variation, the functional relationship governing irradiance distribution in the focal plane of the lens is given below. The graphical depiction of this situation is shown in figure 8 . Note that a linear phase function amounts to a tilt in the wavefront or a change in the direction of arrival vector.

The wave field found in the focal plane of the lens is found to again be a scaled Fourier transform of the wave after modulation by the pupil.

$$
\begin{gathered}
u_{f p}(x, y)=A B_{l} \frac{e^{j \lambda f}}{j \lambda f} q\left(x, y ; \frac{1}{\lambda f}\right)\left\{F F\left[u_{1}^{-}(x, y) \cdot t_{1}(x, y)\right]\right\}_{\xi=x / \lambda f, \eta=y / \lambda f} \\
q(x, y ; a)=e^{j p a\left(x^{2}+y^{2}\right)}
\end{gathered}
$$

$B_{l}=$ constant that represents the optical thickness of the lens at its center and any losses due to reflection or absorption in the lens

The irradiance distribution in the focal plane of the lens is found directly from the squared-modulus of the wave field.

$$
i_{f p}(x, y)=\left|\frac{A B_{l}}{\lambda f}\right|^{2}\left|F F\left[u_{1}^{-}(x, y) \cdot t_{1}(x, y)\right]\right|_{\xi=x / \lambda f, \eta=y / \lambda f}^{2}
$$




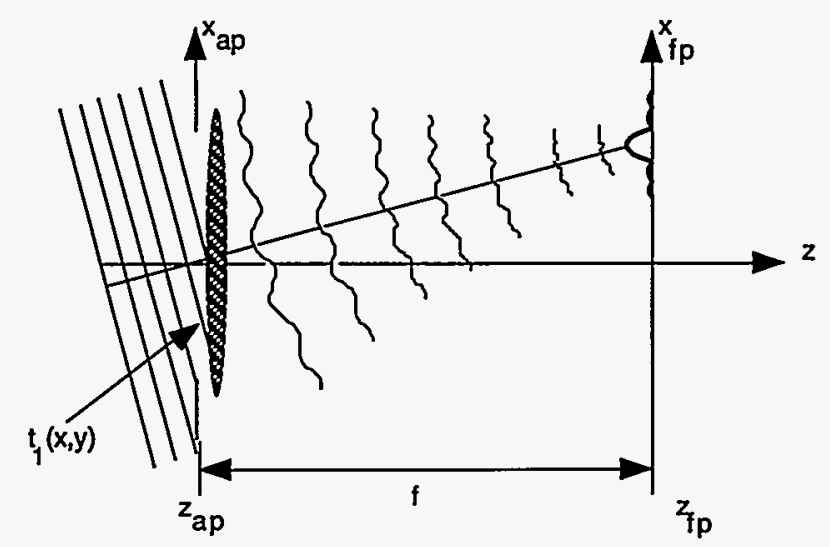

Figure 8: Fresnel Diffraction Geometry with Aperture at the Lens

Using the Fourier-transformation rule of a product, the irradiance can also be represented as the convolution of the Fourier transforms of the wave field and the pupil function,

$$
i_{f p}(x, y)=\left|\frac{A B_{l}}{\lambda f}\right|^{2}\left|F F\left[u_{1}^{-}(x, y)\right] * * F F\left[t_{1}(x, y)\right]\right|_{\xi=x / \lambda f, \eta=y / \lambda f}^{2},
$$

where ** represents the two-dimensional convolution operation.

\section{Monochromatic Diffraction PSF}

If $u_{1}^{-}(x, y)$ is an on-axis, infinite-extent plane wave, (as is the case for long-range laser illumination), then its Fourier transform is a delta function that appears in the focal plane at a location proportional to the $\mathrm{x}$ and $\mathrm{y}$ direction cosines of the normal to the wave, $\gamma_{x}$ and $\gamma_{y}$ (the direction of arrival vector).

$$
\begin{gathered}
i_{\text {laser }}(x, y)=\left|\frac{A B_{l}}{\lambda f}\right|^{2}\left|F F\left[e^{j 2 k\left(\gamma_{x} x+\gamma_{y} y\right)}\right] * * F F\left[t_{1}(x, y)\right]\right|_{\xi=x / \lambda f, \eta=y / \lambda f}^{2} \\
i_{\text {laser }}(x, y)=\left|\frac{A B_{l}}{\lambda f}\right|^{2}\left|\delta\left(\xi-\frac{\gamma_{x}}{\lambda}, \eta-\frac{\gamma_{x}}{\lambda}\right) * * F F\left[t_{1}(x, y)\right]\right|_{\xi=x / \lambda f, \eta=y / \lambda f}^{2}
\end{gathered}
$$

The Fourier-transform of the pupil function will then appear in the focal plane at a point centered on this delta function which is governed by the direction of arrival of the incoming wave. Since the laser appears as an impulse to the system, the squared Fourier-transform of the pupil function represents the impulse response of the system. Therefore, for on-axis, plane-wave laser illumination, in the absence of aberration, the irradiance pattern at the focal plane is the diffraction limited point-spread-function of the system.

$$
p s f_{\text {diff }}(x, y, \lambda)=k_{d} i_{f p}(x, y, \lambda)=k_{d}\left|\frac{A B_{B}}{\lambda f}\right|^{2} \mid F F\left[\left.t_{1}(x, y)\right|_{\xi=x / \lambda f, \eta=y / \lambda f} ^{2},\right.
$$

$k_{d}$ is a constant used to normalize the diffraction PSF to unit area. 
Calculation of the Laser Signal Spatial Frequency Spectrum

The first step necessary to express the analysis of clutter limited SNR in the frequency domain is to determine the signal spectrum in the spatial frequency domain. By examining equation 14 it is possible to derive the spatial frequency content of the laser signal after diffraction by the pupil, $t_{1}(x, y)$. The Fourier transform of the laser impulse response is the frequency content of that signal. Assuming on-axis, plane-wave illumination,

$$
\begin{aligned}
I_{\text {laser }}(\xi, \eta) & =F F\left[i_{\text {laser }}(x, y)\right]=F F\left[\left|\frac{A B_{l}}{\lambda f}\right|^{2}\left|F F\left[t_{1}(x, y)\right]\right|_{\xi=x / \lambda f, \eta=y / \lambda f}^{2}\right] \\
& =\left|\frac{A B_{l}}{\lambda f}\right|^{2} F F\left[\left|T_{1}\left(\frac{x}{\lambda f}, \frac{y}{\lambda f}\right)\right|^{2}\right]
\end{aligned}
$$

where $T_{1}$ is the scaled two-dimensional Fourier transform of the pupil function $\mathrm{t}$.

$$
T_{1}\left(\frac{x}{\lambda f}, \frac{y}{\lambda f}\right)=F F\left\{t_{1}(x, y)\right\}_{\xi=x / \lambda f, \eta=y / \lambda f} .
$$

Using the transform relation for the squared-modulus operation,

$$
|a(x, y)|^{2} \Leftrightarrow \gamma_{F}(\xi, \eta)=A(\xi, \eta) A^{*}(\xi, \eta)
$$

the spatial frequency content of the laser signal after diffraction by the pupil is seen to be the complex autocorrelation of the pupil function, $\gamma_{F}(\xi)$, where the two dimensional cross correlation operation is denoted by

$$
\begin{aligned}
I_{\text {laser }}(\xi, \eta) & =\left|\frac{A B_{l}}{\lambda f}\right|^{2} F F\left[\left|T_{1}\left(\frac{x}{\lambda f}, \frac{y}{\lambda f}\right)\right|^{2}\right] \\
& =\left|\frac{A B_{l}}{\lambda f}\right|^{2}\left(F F\left[T_{1}\left(\frac{x}{\lambda f}, \frac{y}{\lambda f}\right)\right] F F\left[T_{1}\left(\frac{x}{\lambda f}, \frac{y}{\lambda f}\right)\right]\right.
\end{aligned}
$$

$$
I_{\text {laser }}(\xi, \eta)=\left.\left|A B_{l}\right|^{2}\left(t_{1}(x, y) \notin t_{1}(x, y)^{*}\right)\right|_{x=\xi \lambda f, y=\eta \lambda f}
$$

This key result is that the spatial frequency content of the diffracted laser signal as well as the diffraction-limited PSF, is a scaled version of the autocorrelation of the pupil function. Autocorrelation is simply the integral under two copies of the same function as one is translated past the other. 
One Dimensional :

$$
f(x) \propto f(x)=\int_{-\infty}^{\infty} f(\alpha) f^{*}(\alpha-x) d \alpha
$$

Two Dimensional: $\quad f(x, y) f(x, y)=\int_{-\infty}^{\infty} \int_{-\infty}^{\infty} f(\alpha, \beta) f^{*}(\alpha-x, \beta-y) d \alpha d \beta$

This property will allow an intuitive approach for selecting the optimum pupil function to match a system transfer function later in this report. This technique is especially intuitive if the problem is one-dimensional and the pupil is real-valued only (no phase modulation). In this case one can visualize the form of the result relatively easily.

\section{Effects of the Optical Aberrations on the Signal Irradiance Distribution in the Focal Plane}

In the situation where geometric aberrations are present in the system, a similar geometric pointspread-function resulting from these aberrations must also be included to accurately describe the irradiance distribution at the detector. This geometric PSF is convolved with the laser diffraction pattern to yield a smoothed version.

$$
i_{\text {laser }}(x, y)=i_{\text {laser }}(x, y) * * p s f_{g e o}(x, y)
$$

The smoothing of the signal by convolution with the geometric PSF, can be represented as a lowpass filter transfer function $H_{g e o}(\xi, \eta)$ :

$$
H_{g e o}(\xi, \eta)=F F\left(p s f_{g e o}(x, y)\right) .
$$

The spatial-frequency spectra of the irradiance pattern found in the focal plane of the lens due to an input laser signal is then found by,

$$
I_{\text {laser }}(\xi, \eta)=I_{\text {laser }}(\xi, \eta) \cdot H_{g e o}(\xi, \eta) .
$$

\section{Calculation of Clutter Noise Spatial Frequency Spectrum}

The second step in determining the clutter-limited SNR is to represent the background irradiance as the optical system filters it and presents it to the detector array. This irradiance distribution is the clutter-noise against which the system is detecting the signal. Similar relationships to those developed above for signal can be developed to represent the spatial frequency spectrum of this clutter noise.

While the laser is monochromatic in nature, the background flux is broad-band, having its origin in the broad-band emissions of the sun. Thus, to compute the irradiance spatial frequency spectrum seen in the focal plane from the earth background, a polychromatic diffraction PSF must be generated. This PSF can be convolved with the scene distribution to compute the resulting irradiance distribution. 


\section{Polychromatic Diffraction PSF}

While the monochromatic diffraction PSF indicates how the system will respond to a monochromatic point-source, the scene presented to a system is not monochromatic, but essentially broad-band reflected-solar or earth-emitted radiation. Thus the PSF for broad-band sources depends on the source spectrum and the net sensor spectral responsivity. To compute the net polychromatic diffraction PSF requires a weighted integral over the source spectrum and the instrument spectral bandwidth. In this case the source spectrum is reflected solar radiation that can be modeled as a $5900 \mathrm{~K}$ blackbody spectrum (atmospheric attenuation has been neglected for simplicity).

$$
\begin{aligned}
P S F_{\text {poly_diff }}(x, y) & =K \int_{\lambda_{\min }}^{\lambda_{\max }} \phi(\lambda) R(\lambda) I_{f p}(x, y, \lambda) d \lambda \\
& =K \int_{\lambda_{\min }}^{\lambda_{\max }} \phi(\lambda) R(\lambda) \mid F F\left[\left.t_{1}(x, y)\right|_{\xi=x / \lambda f, \eta=y / \lambda f} ^{2} d \lambda\right.
\end{aligned}
$$

$R(\lambda)=$ Net spectral responsivity of optics and detector

$\phi(\lambda)=5900 \mathrm{~K}$ spectral blackbody function

$\mathrm{K}=$ constant to normalize to unit area

This polychromatic diffraction PSF gives the response of the system to a broad-band point source, such as a background scene point. Together with a definition of the scene irradiance distribution, the clutter noise can be computed.

Scene Definition and Clutter Noise Representation Using the Diffraction and Geometric PSF's

A definition is necessary for the scene irradiance distribution that will be used for computing clutter noise. Gaskill ${ }^{6}$ discusses the geometric image irradiance distribution, $i_{\text {scene }}(x, y)$, in the focal plane of the same diffraction limited optical system discussed above, due to an incoherent scene radiance distribution in the object plane, $n_{\text {scene }}(x, y)$.

$$
\begin{gathered}
i_{\text {scene }}(x, y)=\hat{K}\left|B_{l}\right|^{2}\left(\frac{1}{m}\right)^{2} n_{\text {scene }}\left(\frac{x}{m}, \frac{y}{m}\right) \\
\hat{K}=\left(\frac{1}{z_{\text {scene }}}\right)^{2} \iint_{-\infty}^{\infty}\left|t_{1}(x, y)\right|^{2} d x d y \\
z_{\text {scene }}=\text { axial distance to the scene } \\
\mathrm{m}=\text { net system magnification }
\end{gathered}
$$


These results are valid if the aperture stop precedes the lens and forms the entrance pupil of the system. An obvious, but important result is that the image plane irradiance at the detector is proportional to the pupil area, which is determined by the integral in the $\hat{K}$ term. Since differing apertures will have differing areas, the area term will be retained and the other constants grouped into the single constant $K_{\text {scene }}$.

$$
\begin{gathered}
i_{\text {scene }}(x, y)=K_{\text {scene }} A_{\text {pup }} n_{\text {scene }}\left(\frac{x}{m}, \frac{y}{m}\right) \\
A_{\text {pup }}=\iint_{-\infty}^{\infty}\left|t_{1}(x, y)\right|^{2} d x d y \\
K_{\text {scene }}=\left(\frac{\left|B_{l}\right|}{m z_{\text {scene }}}\right)^{2}
\end{gathered}
$$

To arrive at the representation of the clutter signal at the focal plane of the lens, the scaled scene is convolved with the polychromatic-diffraction and geometric-PSF's.

$$
i_{c \text { cluter }}(x, y)=i_{\text {scene }}(x, y) * * p s f_{\text {poly_diff }}(x, y) * * p s f_{g e o}(x, y)
$$

The convolution operations can be thought of as a low-pass filtering of the scene. By Fourier transformation of this relationship, the spatial-frequency spectrum of the clutter is found, as filtered through the optics.

$$
\begin{gathered}
I_{\text {clutter }}(\xi, \eta)=I_{\text {scene }}(\xi, \eta) \cdot F F\left[p s f_{\text {poly_diff }}(x, y)\right] \cdot F F\left[p s f_{g e o}(x, y)\right] \\
I_{\text {clutter }}(\xi, \eta)=I_{\text {scene }}(\xi, \eta) \cdot H_{\text {poly_diff }}(\xi, \eta) \cdot H_{g e o}(\xi, \eta)
\end{gathered}
$$

This background scene signal reaching the detector is unwanted when a system is intended to look for laser signals. It therefore represents 'noise' against which the system must detect. Finding creative methods of rejecting this 'clutter noise' while retaining as much signal as possible is the intent of this report. Comparing the spatial-frequency content of the noise to that of a laser signal will give intuition into possible methods of improving clutter-limited SNR.

To continue the development of an entire system model, it is necessary to develop the relationships that govern how the signal and noise irradiance distributions are processed by the detector array and subsequent electronic subsystems.

\section{Frequency Domain Representation a Detector Matrix Array}

It is important to understand the properties of a detector matrix array as it effects the net system transfer function. For these discussions only the spatial frequency properties of matrix arrays will be examined. While the temporal frequency properties effect the signal content in the detector output, these concepts are beyond the scope of this report. 
A matrix array of rectangular detector elements, shown in figure 9, integrates the scene irradiance presented to it into a ( $\mathrm{n} \times \mathrm{m}$ ) output matrix of numerical values that is intended to represent the irradiance pattern presented to it, $i_{o p t}(x, y)$.

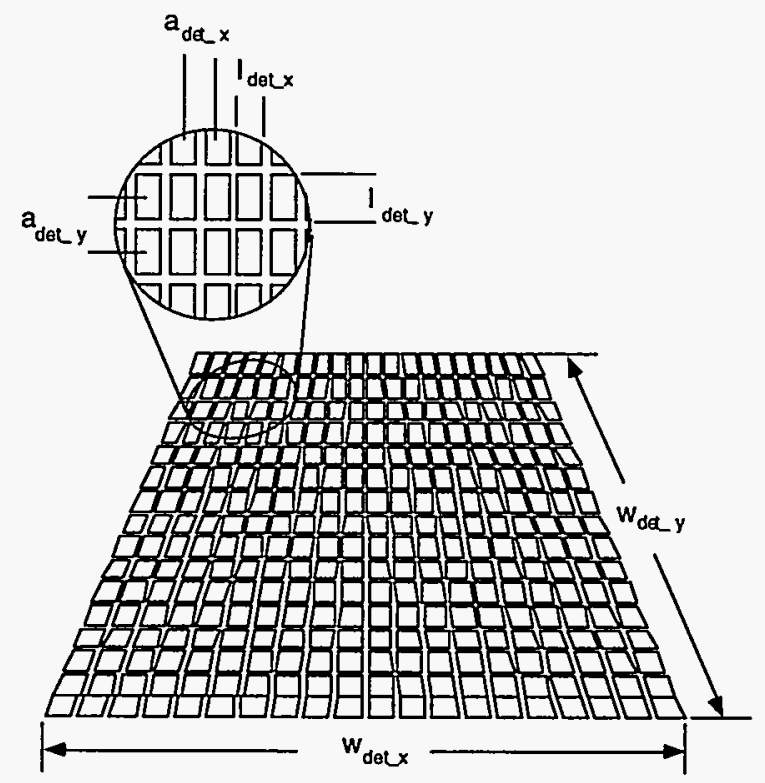

Figure 9: Matrix Array Layout

The detector operation on the irradiance distribution is written as a bounded uniform sampling of the two-dimensional convolution $(* *)$ of the pixel shape with the scene.

$$
\begin{aligned}
& s_{\text {samp }}(x, y)=R_{d} \operatorname{rect}\left(\frac{x}{w_{\text {det_ } x}}, \frac{y}{w_{\text {det_y }}}\right) . \\
& \left\{\frac{1}{a_{\mathrm{det}_{-} x} a_{y}} \operatorname{comb}\left(\frac{x}{a_{\mathrm{det}_{-} x}}, \frac{y}{a_{\mathrm{det}_{-} y}}\right) \cdot\left[i_{\text {opt }}(x, y) * * \operatorname{rect}\left(\frac{x}{l_{\mathrm{det}_{-} x}}, \frac{y}{l_{\mathrm{det}_{-} y}}\right)\right]\right\} \\
& s_{\text {samp }}(x, y) \quad \text { spatial domain detector output signal } \\
& w_{\text {det_}_{-}}, w_{\text {det }_{-} y} \quad \text { maximum linear dimensions of the matrix active area in the } \mathrm{x} \text { and } \mathrm{y} \\
& \text { directions; } \\
& a_{\text {det_}_{-}}, a_{\text {det_}_{-} y} \quad \text { center-to-center spacing of pixel active areas in the } \mathrm{x} \text { and } \mathrm{y} \text { directions; } \\
& l_{x}, l_{y} \quad \text { dimensions of the pixel active areas in the } \mathrm{x} \text { and } \mathrm{y} \text { directions; } \\
& \operatorname{comb}\left(\frac{x}{a}, \frac{y}{b}\right) \quad \text { two dimensional array of unit area delta functions spaced by } \mathrm{a} \text { and } \mathrm{b} \text { in } \\
& \text { the } \mathrm{x} \text { and } \mathrm{y} \text { directions respectively; } \\
& R_{d} \quad \text { detector responsivity (signal/W); }
\end{aligned}
$$


The rect function is defined by Gaskill as follows.

$$
\begin{aligned}
& \operatorname{rect}\left(\frac{x-x_{0}}{b}\right)=\left\{\begin{array}{l}
0,\left|\frac{x-x_{0}}{b}\right|>\frac{1}{2} \\
\frac{1}{2},\left|\frac{x-x_{0}}{b}\right|=\frac{1}{2} \\
1,\left|\frac{x-x_{0}}{b}\right|<\frac{1}{2}
\end{array}\right. \\
& \operatorname{rect}\left(\frac{x-x_{0}}{a}, \frac{y-y_{0}}{b}\right)=\operatorname{rect}\left(\frac{x-x_{0}}{a}\right) \cdot \operatorname{rect}\left(\frac{y-y_{0}}{b}\right)
\end{aligned}
$$

This above operation can be described as follows. The integration of the scene onto the array is represented by the convolution of the rectangular pixel shape with the optically filtered scene. The matrix array output is the value of this convolution evaluated at each of the pixel locations. This is represented by the sifting property of the comb function which samples the convolution to determine the pixel output. A final bounding box of the maximum array dimensions is applied to limit the signal space only to valid pixels.

One note concerning this formulation is necessary. The operation of the matrix array is not a shift invariant system. For example, if an impulse is applied to the input of the system, the output is a single pixel with adjacent zeros. If the same impulse is applied directly between two pixels, the output will be two evenly illuminated pixels. Since the system does not give the same output for an equivalent shifted input, it is not shift-invariant. Because of this property, one transfer function does not apply through out the signal space. To allow a unified approach to analyzing an entire system from a cascaded transfer function approach, will require developing a sampling function representation of the operation of the detector on the scene spatial-frequency spectrum.

By Fourier transformation of equation 35, the frequency domain representation of the detector operation can be shown below.



Applying the Fourier-transform yields 


$$
\begin{aligned}
S_{\text {samp }}(\xi, \eta) & =K \cdot \operatorname{sinc}\left(\xi w_{\text {det_}_{-}}, \eta w_{\text {det_}_{-}}\right) * * \\
& \left\{\left[I_{o p t}(\xi, \eta) \cdot \operatorname{sinc}\left(\xi l_{\text {det_}_{-}}, \eta l_{\text {det }_{-} y}\right)\right] * * \operatorname{comb}\left(\xi a_{\text {det_x }_{-}}, \eta a_{\text {det_}_{-}}\right)\right\}
\end{aligned}
$$

where

$$
K=R_{d}\left(w_{\operatorname{det}_{-} x} w_{\operatorname{det}_{-} y}\right)\left(l_{\operatorname{det}_{-} x} l_{\operatorname{det}_{-} y}\right),
$$

and the sinc function is defined by Gaskill as follows.

$$
\begin{gathered}
\operatorname{sinc}\left(\frac{x-x_{0}}{b}\right)=\frac{\sin \pi\left(\frac{x-x_{0}}{b}\right)}{\pi\left(\frac{x-x_{0}}{b}\right)} \\
\operatorname{sinc}\left(\frac{x-x_{0}}{a}, \frac{y-y_{0}}{b}\right)=\operatorname{sinc}\left(\frac{x-x_{0}}{a}\right) \cdot \operatorname{sinc}\left(\frac{y-y_{0}}{b}\right)
\end{gathered}
$$

As is typically the case, the matrix contains a large number of pixels so

$$
w_{\text {det_ } x} \gg l_{\text {det_ } x} \text { and } w_{\text {det_}_{-} y}>l_{\text {det_}_{-}},
$$

and therefore

$$
\frac{1}{w_{\text {det_}_{-} x}}<\frac{1}{l_{\text {det_}_{-}}} \text {and } \frac{1}{w_{\text {det_}_{-} y}}<\frac{1}{l_{\text {det_}_{-} y}} .
$$

Thus the sinc function associated with the total width and length of the matrix approximates a delta function relative to the sinc associated with the pixel dimensions. In this case the transfer function is dominated by the latter and the sinc function associated with the overall array dimensions can be removed.

$$
S_{\text {samp }}(\xi, \eta)=\left(R_{d} l_{\mathrm{det}_{-} x} l_{\mathrm{det}_{-} y}\right)\left\{\left[I_{o p t}(\xi, \eta) \cdot \operatorname{sinc}\left(\xi l_{\mathrm{det}_{-} x}, \eta l_{\mathrm{det}_{-} y}\right)\right] * \operatorname{comb}\left(\xi a_{\mathrm{det}_{-} x}, \eta a_{\mathrm{det}_{-} y}\right)\right\}
$$

This result is consistent with traditional treatments of sampled systems. Its interpretation is that the spatial frequency spectrum of the input irradiance function, after spatial filtering by integration onto the array, is replicated in frequency space at every integer multiple of the detector sampling frequency in each direction.

$$
\xi_{n}=\frac{n}{a_{\mathrm{det}_{-} x}}, \eta_{n}=\frac{n}{a_{\mathrm{det}_{-} y}}
$$

This is effect graphically depicted in figure 10 .

If the sampling frequency of the array does not exceed the bandwidth of the signals expected at the detector array, the zero and first order spectrums overlap and add together or are 'aliased' (see also figure 10). This prevents the original signal from being transmitted without distortion or error. 


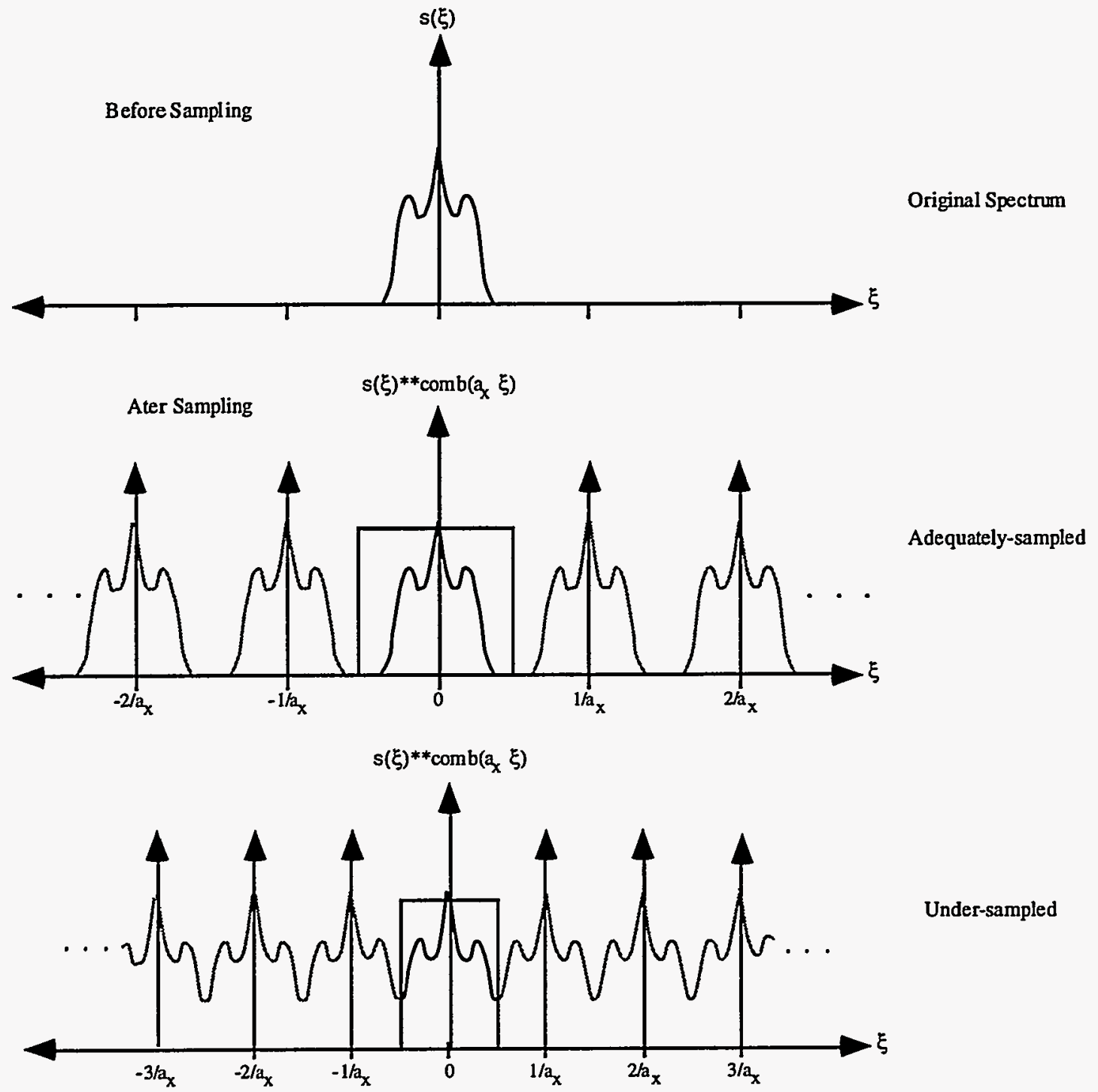

Figure 10: Aliasing in Sampled Systems

Aliasing directly modifies the spectrum of the input signal. Typically it manifests as an increase in high-frequency signal. Depending on the signal processing technique used, the effects will directly translate into a measurement error. By adhering to the Nyquist theorem ${ }^{7}$, where the detector spatial sampling frequency is twice that of the largest expected signal frequency, this can be avoided.

7 Ibid., p. 269 
Equation 43 can be written as a sampling operator $\Phi_{\text {samp }}$ shown below.

$$
\begin{aligned}
\Phi_{\text {samp }}\left(I, a_{\mathrm{det}_{-}}, a_{\mathrm{det}_{-} y}, l_{\mathrm{det}_{-} x}, l_{\mathrm{det}_{-} y}\right)=\left(R_{d} l_{\mathrm{det}_{-} x} l_{\mathrm{det}_{-} y}\right) \\
\cdot\left\{\left[I \cdot \operatorname{sinc}\left(\xi l_{\mathrm{det}_{-} x}, \eta l_{\mathrm{det}_{-} y}\right)\right] * * \operatorname{comb}\left(\xi a_{\mathrm{det}_{-} x}, \eta a_{\mathrm{det}_{-} y}\right)\right\}
\end{aligned}
$$

The signal output can now be written using this sampling operator.

$$
S_{\text {samp }}(\xi, \eta)=\Phi_{\text {samp }}\left(I_{\text {opt }}(\xi, \eta), a_{\mathrm{det}_{-} x}, a_{\mathrm{det} \__{-}}, l_{\mathrm{det}_{-},}, l_{\mathrm{det}_{-} y}\right)
$$

With general functions written for the operation of the optical system and the detector array, a simplified approach will be developed to represent the operation of the analog and digital electronics on the signal that is generated by the detector array.

\section{Simple Analog Filter Transfer Function}

The signal leaving the detector array is amplified and filtered by analog amplifiers and active and passive analog filters. It is not the purpose of this report to provide a essay into these techniques in detail. For brevity, the system will be assumed to be linear and shift-invariant, and can be represented as a simple transfer function with linear phase response.

The signal spectrum must have a change of variables from the spatial frequency $(\xi)$ to temporal frequency $(\omega)$ domains.

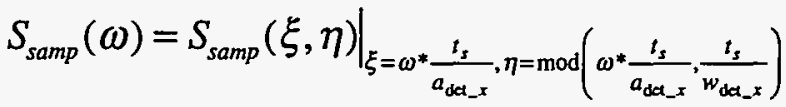

$$
\begin{aligned}
& t_{s}=\text { temporal digitization rate (s) }
\end{aligned}
$$

A simple representation of the analog filter transfer function, $H_{\text {analog }}(\omega)$, can be applied to determine the signal spectrum after filtering.

$$
S_{\text {samp }}(\omega)=H_{\text {ana log }}(\omega) \cdot S_{\text {samp }}(\omega)
$$

If the electronic designer has correctly tailored the response, the transfer function for the amplifiers will have relatively flat response over the entire expected frequency spectrum. In general this may not be the case and the amplifier response must be included. For this report, the transfer function will be assumed to be dominated by filtering intentionally placed in the design to tailor the response and achieve the best signal-to-noise ratio.

\section{Simple Digital Filtering Transfer Function}

The analog signal produced by the amplifier and the associated analog filters is usually fed to an aanalog-to-digital (A/D) converter for quantization and readout for processing. Digital filters can be implemented to manipulate the data after it has been quantized. As for the analog filtering, the digital filtering is assumed to be linear and shift-invariant, and can be represented as a simple transfer function of sample frequency space variable $\zeta, H_{\text {dig }}(\zeta)$, with linear phase response. 


$$
S^{\prime \prime}{ }_{s a m p}(\zeta)=\left.H_{d i g}(\zeta) \cdot S_{s a m p}(\omega)\right|_{\omega=\frac{\zeta}{t_{s}}}
$$

For simplicity, the digitization rate is assumed to be exactly the pixel readout rate. This is generally true except in systems where over-sampling of the analog signal is deemed necessary by the designer.

\section{Signal-to-Noise Ratio Analysis for Laser Detection Against Clutter Noise} Signal-to-Noise Ratio, SNR, for a given input and noise level is a figure of merit that allows comparisons between competing systems. To accurately develop a measure of the clutter-limited SNR, the net transfer function for both laser signal and clutter noise will be summarized from the above discussion. Armed with these results, the slit-grating concept will be analyzed for SNR for a given source and a given background scene. The SNR for similar systems with differing aperture functions will then be analyzed.

\section{Total Transfer Function}

The total system transfer function can be derived by cascading the element transfer functions, scaling the results into a common space, and computing the net transfer function. The simplest realm to do the net transfer function is in the sample and sample-frequency domains, where detection processes will be applied.

For applicability to the following analysis of the slit-grating concept, the system will be considered one-dimensional in $\mathrm{x}$, uniform in $\mathrm{y}$, and all the component transfer functions will be expressed in one dimensional form, with all results translated into the sample frequency domain. This one dimensional representation will be shown later to be applicable to the analysis of the slit-grating concept.

$$
S_{\text {samp }}(\zeta)=H_{\text {dig }}(\zeta) \cdot\left[H_{\text {analog }}(\omega) \cdot\left\{\Phi_{\text {samp }}\left(I(\xi), a_{\text {det_ } \left.\_, l_{\text {det_ }-x}\right)}\right\}_{\xi=\omega^{*} \frac{t_{s}}{a_{\text {data }}}}\right]_{\omega=\frac{\zeta}{t_{s}}}\right.
$$

This allows an end-to-end transfer function to be written that operates on an optical signal $I(\xi)$, either signal or noise, which is presented to the detector array.

\section{RMS Signal-to-Noise Ratio Definition}

To compare systems of differing pupil functions, a figure of merit must be given that is related to the RMS signal-to-noise ratio that a particular system can achieve for a given input. Such a figure of merit is $\alpha$, which is defined as the RMS signal power after filtering divided by the RMS noise power ${ }^{8}$ after filtering. This allows constants in frequency, for both signal and noise, to be neglected. Thus only terms that vary in frequency, or vary with the aperture function, will be retained in the signal and noise formulations. As long as the input signal and the background scene are constant between systems, $\alpha$ is a valid unit of comparison.

${ }^{8}$ Claude S. Linquist, Adaptive and Digital Signal Processing (Steward and Sons, 1989), p. 296 


$$
\alpha=\frac{R M S \text { Signal }}{R M S \text { Clutter Noise }}=\frac{\sqrt{\int_{z=0}^{0.5}\left|S_{\text {samp }}^{\text {sig }}(\zeta)\right|^{2} d \zeta}}{\sqrt{\int_{z=0}^{0.5}\left|S_{\text {samp }}^{\text {noise }}(\zeta)\right|^{2} d \zeta}}
$$

For convenience, the input signal and noise functions will be defined as follows for all systems.

Signal: $u_{s i g}^{-}(x)=1 e^{j 2 k} \quad$ unit amplitude, on-axis, monochromatic plane wave Noise: $i_{\text {scene }}(x)=K_{\text {scene }} A_{\text {pup }} N_{\text {scene }}\left(\frac{x}{m}\right)$ scaled scene radiance function

The optical system will be considered lossless $\left(B_{l}=0\right)$ so the leading coefficient of the signal calculation $\left|A B_{l}\right|$ is unity and can be dropped from the formulations. Now the signal and noise functions can be expressed.

\section{System Response to Laser Signal}

The spatial-frequency representation of the laser signal presented to the detector array can be found using equations 20 and 25.

$$
I_{\text {laser }}^{\text {net }}(\xi)=\left(t_{1}(\xi \lambda f) \nless t_{1}(\xi \lambda f)^{*}\right) \cdot H_{g e o}(\xi)
$$

The functional form of the output signal function for the system can then be written using equation 50.

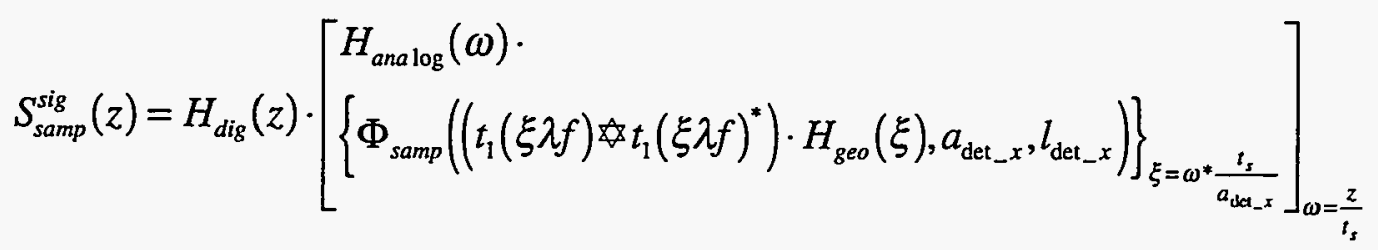

\section{System Response to Clutter Noise}

The response of the system to noise is similar except the scene irradiance is filtered by the polychromatic-diffraction PSF. The response of the system to noise can be directly written from equation 50 .

$$
\begin{aligned}
I_{c l u t e r}^{\text {net }}(\xi) & =K_{\text {scene }} A_{\text {pup }} F F\left[n_{\text {scene }}\left(\frac{x}{m}\right)\right] \cdot H_{\text {poly_diff }}(\xi) \cdot H_{g e o}(\xi) \\
& =K_{\text {scene }} A_{\text {pup }} m N_{\text {scene }}(m \xi) \cdot H_{\text {poly_diff }}(\xi) \cdot H_{g e o}(\xi)
\end{aligned}
$$

The functional form of the output noise function for the system can then be written. 


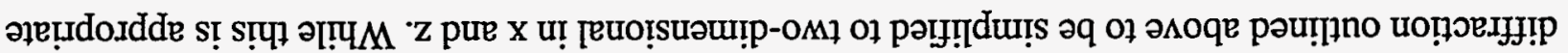

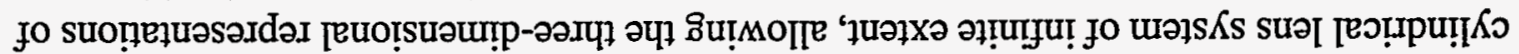

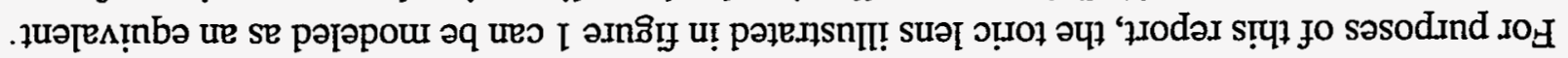



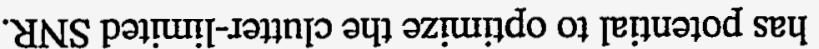



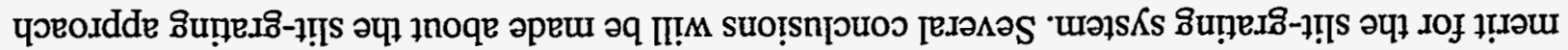

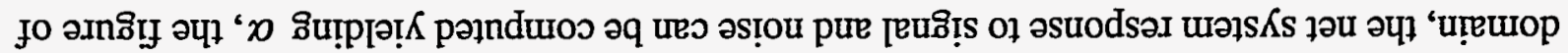



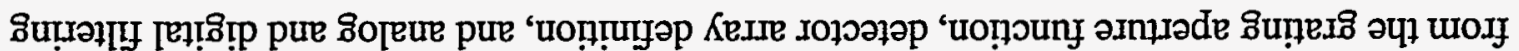

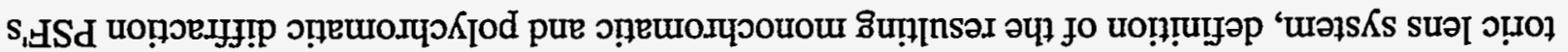

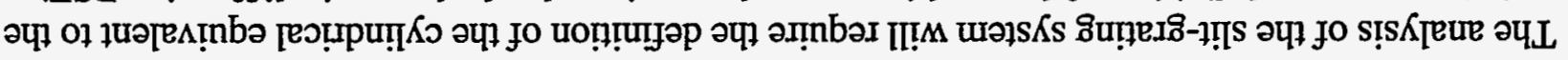

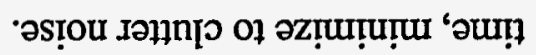

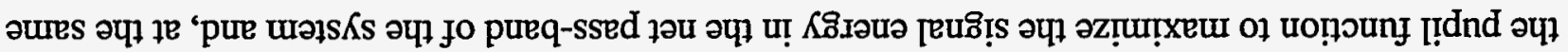

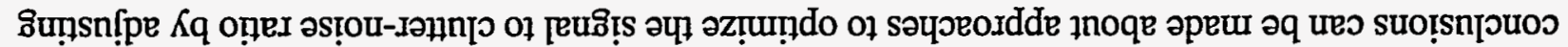

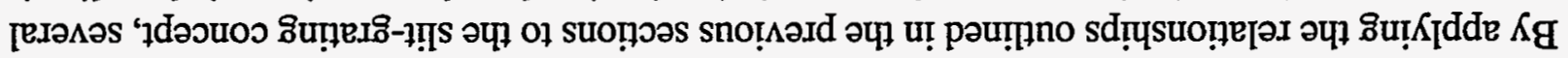

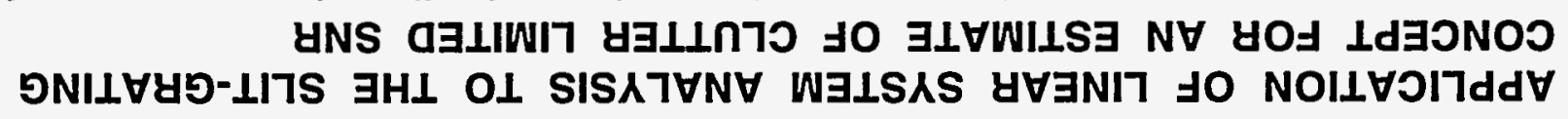

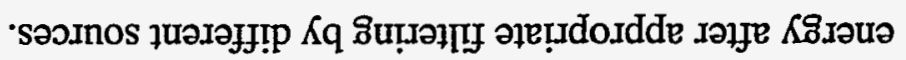

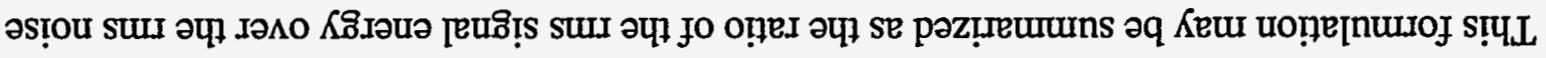



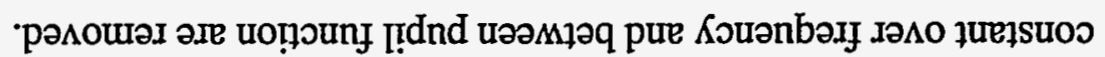

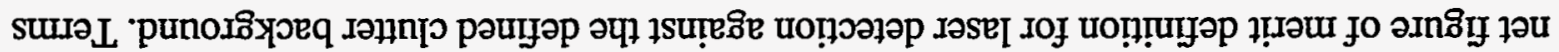

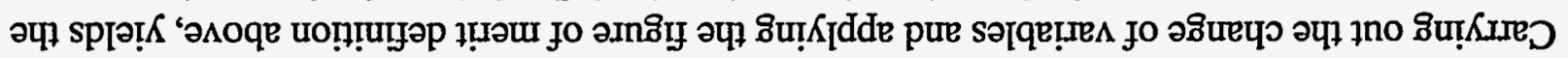
yNS pəI!U!

(s)

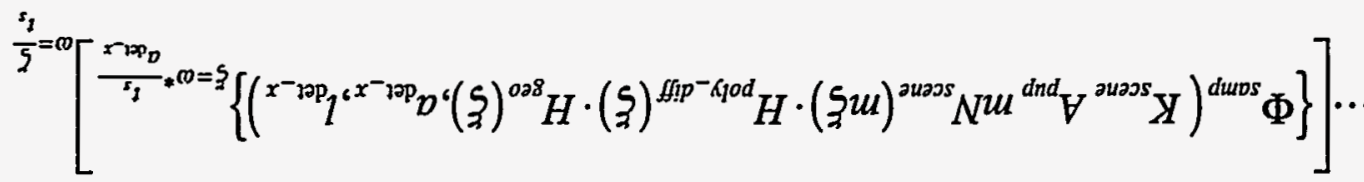

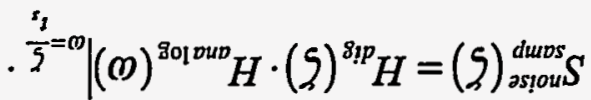


for the toric lens system, it also helps to visualize the optimization because the spatial frequency variations are only in the $\mathrm{x}$-direction.

There are two representations of the cylindrical equivalent of the toric lens system. The first would be to represent the system as a lens with the effective focal length of the toric lens and an exit pupil which is a scaled version of the aperture function. This is the more traditional approach to analyzing optical systems, but does not directly fit with the formalism presented equation 9. A more appropriate equivalent is the case of plane-wave illumination of an aperture in front of a positive lens which is the basis for development of equation 9. In this case the aperture does not need to be scaled and the back-focal-length of the lens is used. This latter equivalent will be used and shown to provide correlation with measured data. With this assumption, the cylindrical equivalent focal length can be used. The equivalent system is shown below.



Figure 11: Cylindrical Equivalent to the Toric Lens

\section{Slit-Grating Pupil Function}

With this equivalent system, the aperture function in equation 9 is the Ronchi-ruling grating function depicted in figure 2 . Formally this can be written as shown below.

$$
t_{1}(x)=\operatorname{rect}\left(\frac{x}{\left(n_{l}+1\right) \cdot a_{g r a t_{-} x}}\right) \cdot\left(\operatorname{rect}\left(\frac{x}{l_{\text {grat_ }} x}\right) * \frac{1}{a_{\text {grat } x}} \operatorname{comb}\left(\frac{x}{a_{g_{\text {grat }} x}}\right)\right)
$$

This is simply a series of rectangular transmitting regions of width $l_{g r a t_{-}}$, spaced $a_{g r a t_{-} x}$ apart, and bounded by a total number of lines, $n_{l}$. For the slit grating concept, these parameters are as follows.

$$
\begin{gathered}
l_{g r a t_{-} x}=3.33 \mu \mathrm{m} \\
a_{g r a t_{-} x}=6.66 \mu \mathrm{m} \\
n_{l}=45
\end{gathered}
$$

\section{Slit-Grating Monochromatic Diffraction PSF and Signal Spectrum} Using equation 57 and equation 11, the form of the monochromatic diffraction PSF of the slitgrating concept can be directly computed. Assuming the signal wave defined on page 21 is the 
laser signal, and that the losses in the lens can be neglected because they will be common to both signal and noise in the SNR calculation, the irradiance in the focal plane can be written as shown below.

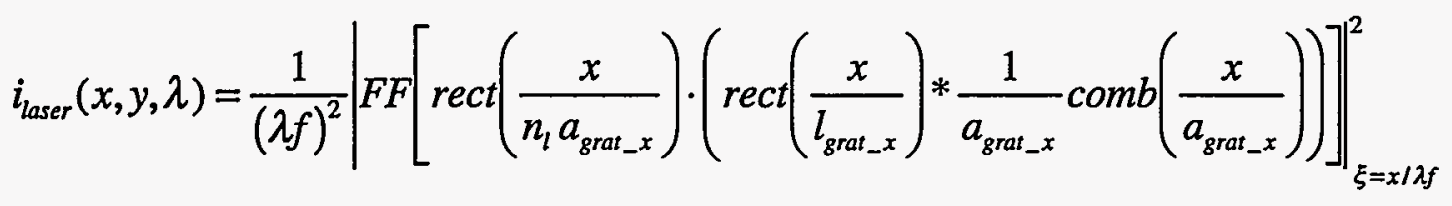

$$
\begin{aligned}
& =\left(\frac{n_{l} l_{\text {grat }} x}{\lambda f}\right)^{2}\left|\sin c\left(\frac{n_{l} a_{\text {grat } x} x}{\lambda f}\right) *\left(\sin c\left(\frac{l_{\text {grat }} x}{\lambda f}\right) \cdot a_{\text {grat } x} \operatorname{comb}\left(\frac{a_{\text {grat }} x}{\lambda f}\right)\right)\right|^{2}
\end{aligned}
$$

As discussed in equation 15, the diffraction psf can be computed by normalizing this relationship to unit area.

To show the correct spatial scaling of the diffraction pattern has been accomplished, the theoretical diffraction PSF for a $1.06 \mu \mathrm{m}$ laser is plotted in figure 12 relative to a measured diffraction pattern at the same wavelength from an actual slit-grating/toric lens combination. Keep in mind the measured diffraction pattern has the lens geometric PSF and the detector pixel functions convolved with it causing the spreading and decreased peak value seen in the data. The lateral scaling error seen between the theoretical and the actual data is slight, probably due to a slight difference between the actual focal length of the toric lens and the theoretical value.

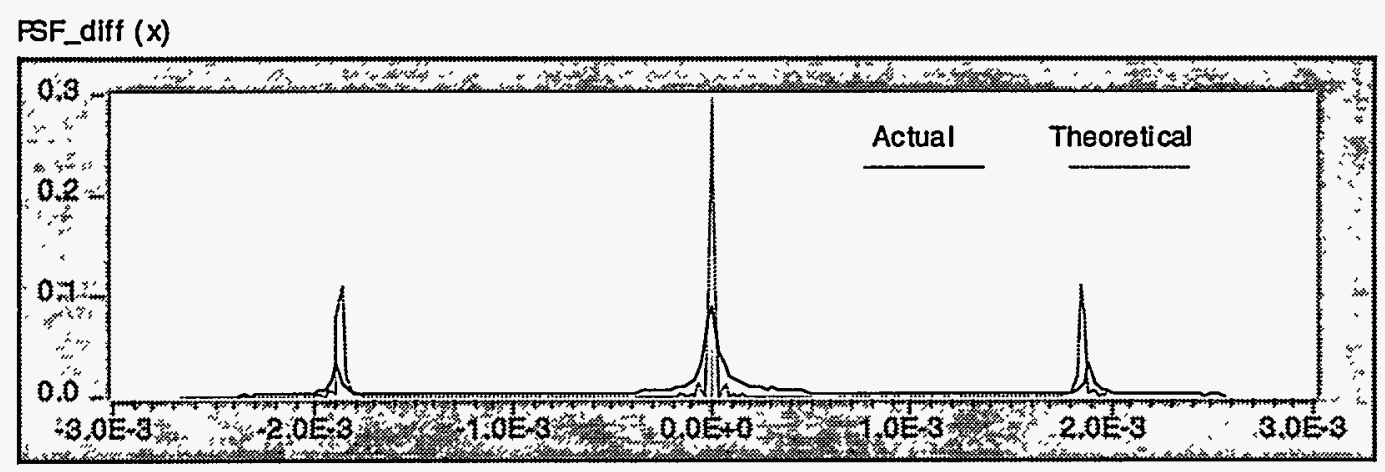

$x(m)$

Figure 12: Slit Grating Monochromatic Diffraction PSF at $\lambda=1.06 \mu \mathrm{m}$

The more important representation of the laser signal for this analysis is its spatial frequency spectrum. The signal amplitude spectrum is calculated from equation 20 as the autocorrelation of the slit-grating pupil function. The spatial frequency spectrum is translated into the sample frequency space by the change of variables $\xi=\zeta / a_{\text {det_ }_{-} x}$ from equation 55 . 


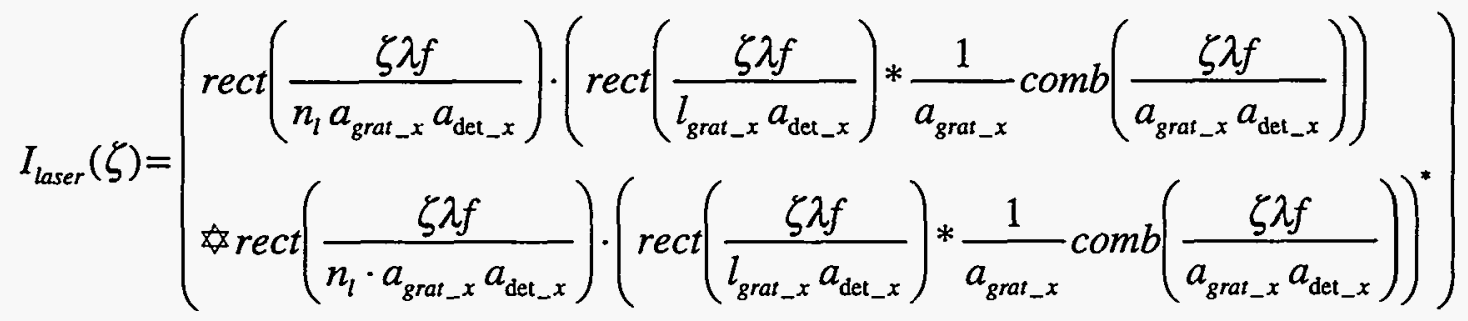

$$
\begin{aligned}
& =\left[n_{l} l_{\text {grat_x }}\right] \cdot\left(\operatorname{tri}\left(\frac{\zeta \lambda f}{l_{g r a t_{-} x} a_{\text {det_x }_{-}}}\right) *\left(\operatorname{tri}\left(\frac{\zeta \lambda f}{n_{l} \cdot a_{\text {grat_ }} a_{\text {det_ }_{-}}}\right) \cdot \frac{1}{a_{g r a t_{-} x}} \operatorname{comb}\left(\frac{\zeta \lambda f}{a_{g r a t_{-} x} a_{\text {det_x }_{-}}}\right)\right)\right)
\end{aligned}
$$

The tri function is defined by Gaskill as shown below.

$$
\operatorname{tri}\left(\frac{x-x_{0}}{b}\right)=\left\{\begin{array}{l}
0,\left|\frac{x-x_{0}}{b}\right| \geq 1 \\
1-\left|\frac{x-x_{0}}{b}\right|,\left|\frac{x-x_{0}}{b}\right|<1
\end{array}\right.
$$

As shown in figure 14, the spatial frequency spectrum of the laser signal can easily be seen as the area under two copies of the slit-grating pupil function as one is translated past the other.

\section{Geometric PSF}

The typical measured geometric PSF for the toric lens system has a total-width, half-power point of 2.0 pixels. The model for the geometric PSF will be a gaussian as defined below which has similar half-power width.

$$
p s f_{\text {geo }}(x)=\frac{1}{2.13 a_{\text {det_ } x}} \exp \left[-\pi\left(\frac{x}{2.13 a_{\text {det_ }_{-}}}\right)^{2}\right]
$$

The filtering properties of the geometric PSF are again found from the magnitude of the Fourier transform of the PSF and substituting $\xi=\zeta / a_{\text {det_x }}$.

$$
\left|H_{g e o}(\zeta)\right|=\exp \left[-\pi(2.13 \zeta)^{2}\right]
$$

\section{Linear Detector Array}

The linear detector array used for the optimization in this report is a unit responsivity 1024 pixels, $2 / 3$ fill-factor linear array with $30 \mu \mathrm{m}$ pixel pitch.

$$
\begin{gathered}
R_{d}=1 \\
l_{\text {det_x }}=20 \mu \mathrm{m} \\
a_{\text {det_x }}=30 \mu \mathrm{m}
\end{gathered}
$$




$$
w_{\text {det_ } x}=1024 * a_{\text {det_x }}=30.72 \mathrm{~mm}
$$

From a filtering point of view in equation 38, the filtering effect of the length of the array appears as a sinc function of width $1 / 1024$ relative to that resulting from the finite length of pixel. The relative sizes of the two sinc functions cause their convolution to be dominated by the wider of the two - that associated with the pixel dimensions. Therefore a one-dimensional form of equation 43 is valid.

$$
\Phi_{\text {samp }}\left(I(\xi), a_{\mathrm{det}_{-} x}, l_{\mathrm{det}_{-} x}\right)=\left(R_{d} \frac{l_{\mathrm{det}_{-} x}}{a_{\mathrm{det} \__{-}}}\right)\left\{\left[I(\xi) \cdot \sin c\left(\xi l_{\mathrm{det}_{-} x}\right)\right] * a_{\mathrm{det}_{-} x} \operatorname{comb}\left(\xi a_{\mathrm{det}_{-} x}\right)\right\}
$$

A change of variables in equation 43 is made to convert the spatial frequency representation of the array operation to the sample frequency domain, $\xi=\frac{\zeta}{a_{\text {det_ }_{-}}}$.

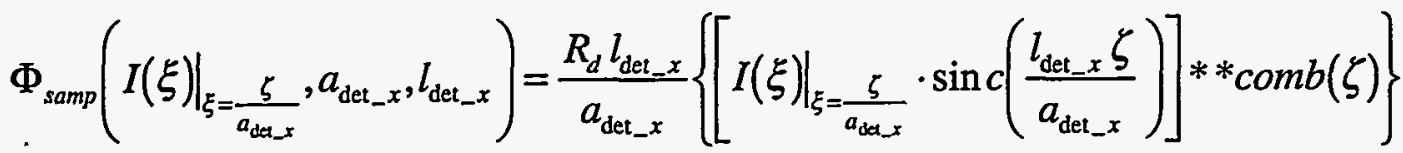

$$
\begin{aligned}
& =0.666\left\{\left[\left.I(\xi)\right|_{\xi=\frac{\zeta}{a_{d c t-1}}} \cdot \sin c\left(\frac{\zeta}{1.5}\right)\right] * * \operatorname{comb}(\zeta)\right\}
\end{aligned}
$$

\section{Analog and Digital Filtering}

For simplicity, all filters that were developed for use with the slit-grating concept will be represented in their digital equivalent. The two primary types of filtering were prequantization filtering, and optimal filtering.

Simple passive high-pass analog filtering techniques were applied to the detector video output prior to quantization in the $\mathrm{A} / \mathrm{D}$ converter, to limit the low-frequency excursions of background signal, reserving the maximum dynamic range for the signal. The sample-space equivalent of this analog 'prequantization filter' used is shown in equation 72. Its frequency response is shown in figure 13.

$$
\mathrm{y}_{\mathrm{pq}}(\mathrm{n})=.904[\mathrm{x}(\mathrm{n})-\mathrm{x}(\mathrm{n}-1)+\mathrm{y}(\mathrm{n}-1)]
$$

Additional filtering was required after quantization which optimized detection of the signal in the presence of the remaining background. Analysis showed that the background signal possessed a $1 / \xi^{3 / 2}$ spectral rolloff. Assuming a two pixel wide signal blur an 'optimal filter' was designed to maximize the signal output while quenching the background. The sample-space representation of this filter is shown in equation 73. The magnitude response of the optimal filter is shown in figure 13.

$$
y_{\text {opt }}(n)=-x(n-3)+x(n-2)+x(n-1)-x(n)
$$




\section{Computation of Net Signal Amplitude Spectrum}

The net laser signal can be computed by overlaying the magnitude transfer functions for the geometric PSF, the array pixels, the prequantization filter and the optimal filter to generate a net system filter function. This overlay is illustrated in figure 13 below. One can immediately see that the optimum signal output would occur when the signal spatial frequency spectrum had its maximum energy placed under the peak in the net filter function at $\zeta=0.2$. When the net system transfer function is applied to the laser signal, the net output signal spectrum is found. Integrating under the net signal spectrum in figure 14 yields the total signal amplitude which will be used in the calculation of $\alpha$, the figure of merit.

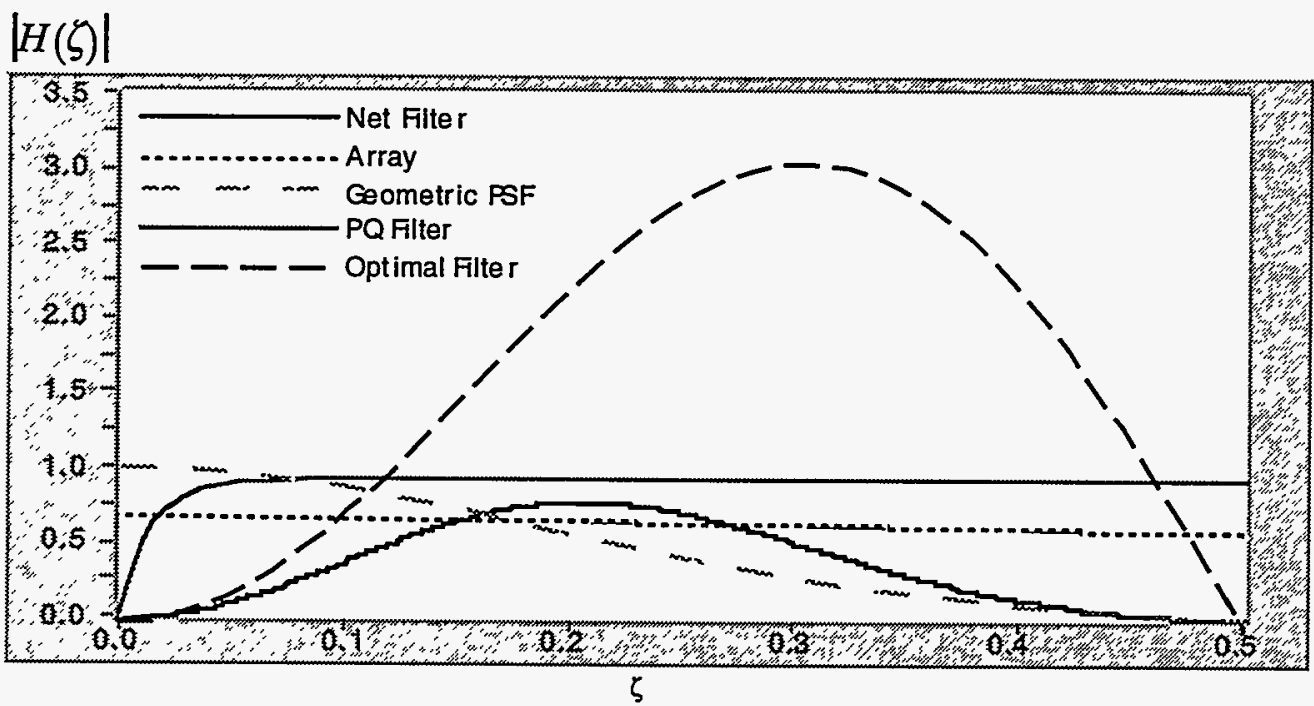

Figure 13: Net System Transfer Function.

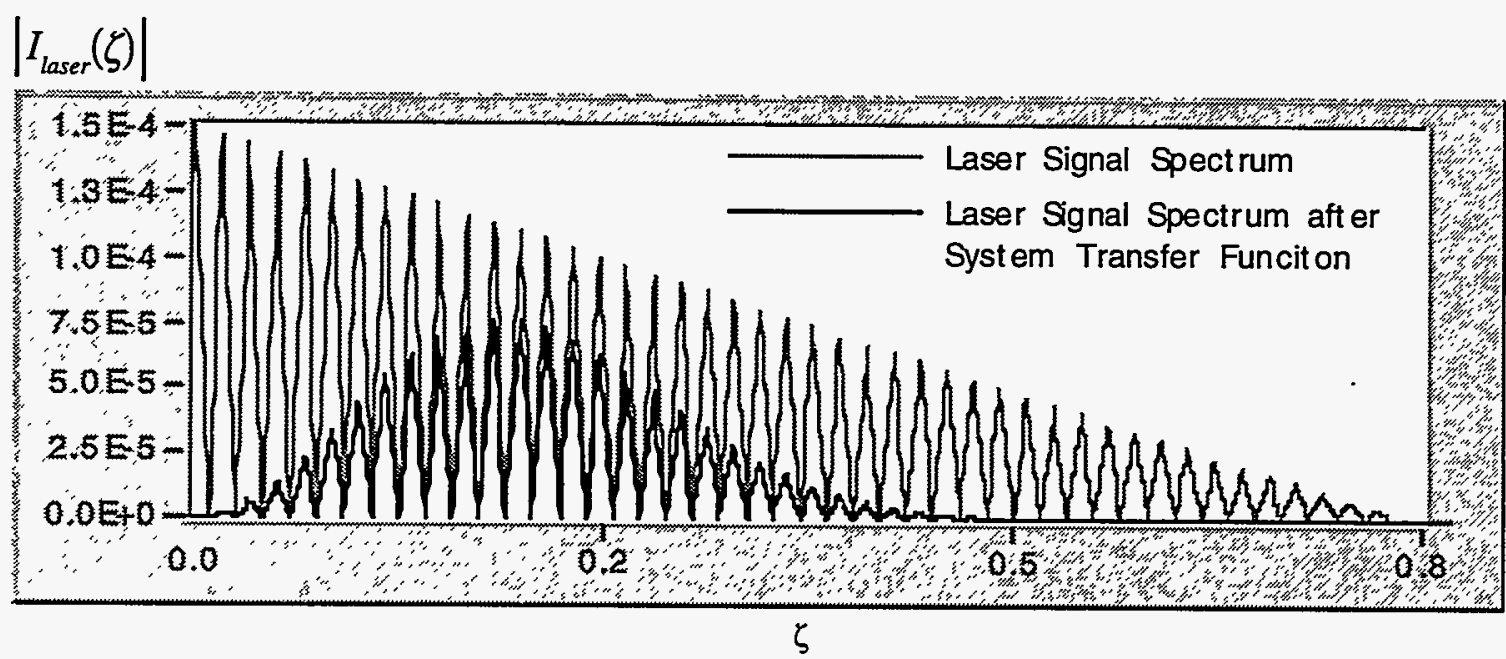

Figure 14: Laser Signal Amplitude Spectrum at $1.06 \mu \mathrm{m}$ Before and After System Transfer Function 


\section{Computation of Net Clutter Noise Amplitude Spectrum}

\section{Polychromatic Diffraction PSF for the Slit-Grating Concept}

To allow the computation of the total clutter noise after filtering by the system, the polychromatic diffraction PSF must be computed for the slit grating design using a formulation similar to equation 26. The detector responsivity curve used is a typical silicon photodiode curve. The blackbody source is assumed to be a 5900K scene which is typical of reflected earth albedo. Since the slitgrating acts like a spectrometer, the shape of the first-order peaks follows the shape of the product of the blackbody curve and the detector responsivity curve, see figure 15 . The numerical integration technique used to determine this function only resolves to the size of the detector pixel. While the actual pattern might have slightly sharper features, it is assumed that this pattern is adequate for these calculations.

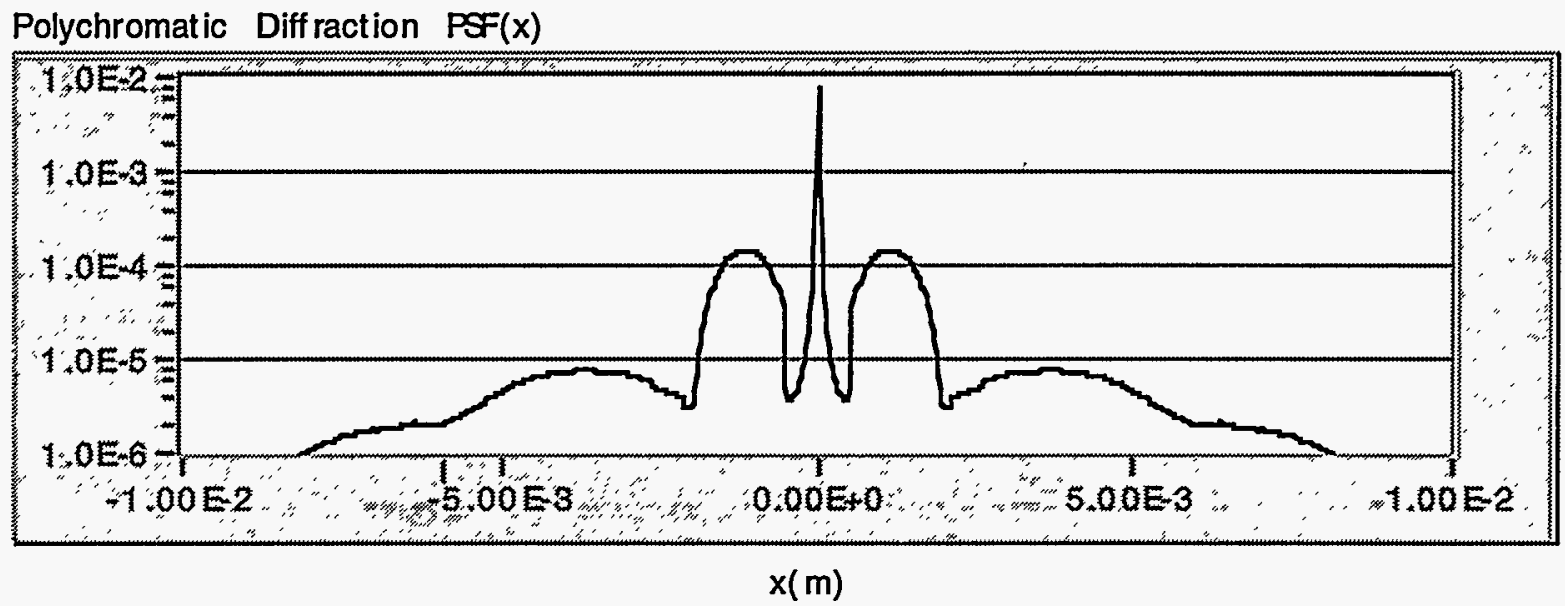

Figure 15: Slit-Grating Polychromatic Diffraction PSF

As mentioned previously, this polychromatic PSF acts as a filter on the background scene. The magnitude response of this filtering is found from the magnitude of the Fourier-transform of the polychromatic diffraction PSF. Again, a change of variables expresses the transfer function in terms of sample frequency, $\zeta$, as shown below in figure 17.

\section{Scene Data Used as a Source for Clutter Noise}

An earth-looking scene of scale similar to the scene this system would see on orbit is illustrated in figure 16. To use this scene as a valid source for clutter noise in the system, it should have a relative scale that would be equivalent to that which the sensor would see on orbit and have adequate sampling to represent the maximum spatial frequency content the sensor can detect. These scenes are captured from a similar orbital altitude that the system was intended to fly at and cover approximately the same angular dimensions as the angular field of view of the sensor. Since the scenes have 1080 samples over their width and length and the detector resolution is 1024 samples over its entire length, this scene will be adequately sampled to represent an actual scene. The amplitude of the scene is scaled proportional to the area of the entrance pupil of the sensor, which 
is proportional to the number of transmitting zones in the grating $n_{l} l_{x}$, and the lateral scale (m) is adjusted to exactly fill the length of the detector array.

$$
i_{\text {scene }}(x) \propto A_{\text {pup }} N_{\text {scene }}\left(\frac{x}{m}, \frac{y}{m}\right) \propto n_{l} l_{x} N_{\text {scene }}\left(\frac{x}{m}, \frac{y}{m}\right)
$$

This and other scenes were analyzed to determine the average scene amplitude spectrum along one axis in the scene. Since the slit-grating system only resolves along one axis, the spectrum along one axis can be used as the input to the model developed here as a sample of the expected scene spectrum. That average spectrum of this scene in the horizontal direction is shown in figure 17. Notice that the scene spectrum has an inverse frequency relationship as was assumed in the design of the 'optimal' filter.



Figure 16: Sample Scene Data

To compute the actual signal spectrum that would be seen at the detector, this scene spectrum must be multiplied by the magnitude transfer function for polychromatic diffraction PSF. The resulting 
spectrum can then be multiplied by the net system transfer function to compute the clutter noise spectrum and the RMS clutter noise. This is shown in figure 17.

\section{Computation of $\alpha$ for the Slit-Grating Design}

With the RMS signal and clutter noise values computed, the figure of merit, $\alpha$, can be calculated. This value alone has no meaning except that it is directly proportional to the clutter limited SNR, and will only be of value as a mark of by which systems with differing pupil functions are compared. The figure of merit for the slit-grating concept was computed over the wavelength band of a typical silicon photodiode sensor spectral response curve to give an indication of the variation of the figure of merit over wavelength. This curve will be useful to determine the relative improvement of proposed systems relative to the slit-grating concept as the signal wavelength varies.

\section{ANALYSIS OF SLIT-GRATING CONCEPT FOR DETERMINING OPTIMUM PUPIL FUNCTION}

The intent of this research was to investigate techniques by which the clutter-noise-limited minimum detectable irradiance levels of the slit-grating concept laser sensor could be reduced by modifying the form of the pupil function from a slit-grating, to some more general function. By examining the laser signal spectrum of the slit-grating concept, a clue is found that indicates what modifications might produce an improvement in SNR.

\section{$\left|I_{\text {scente }}(\zeta)\right|$.}

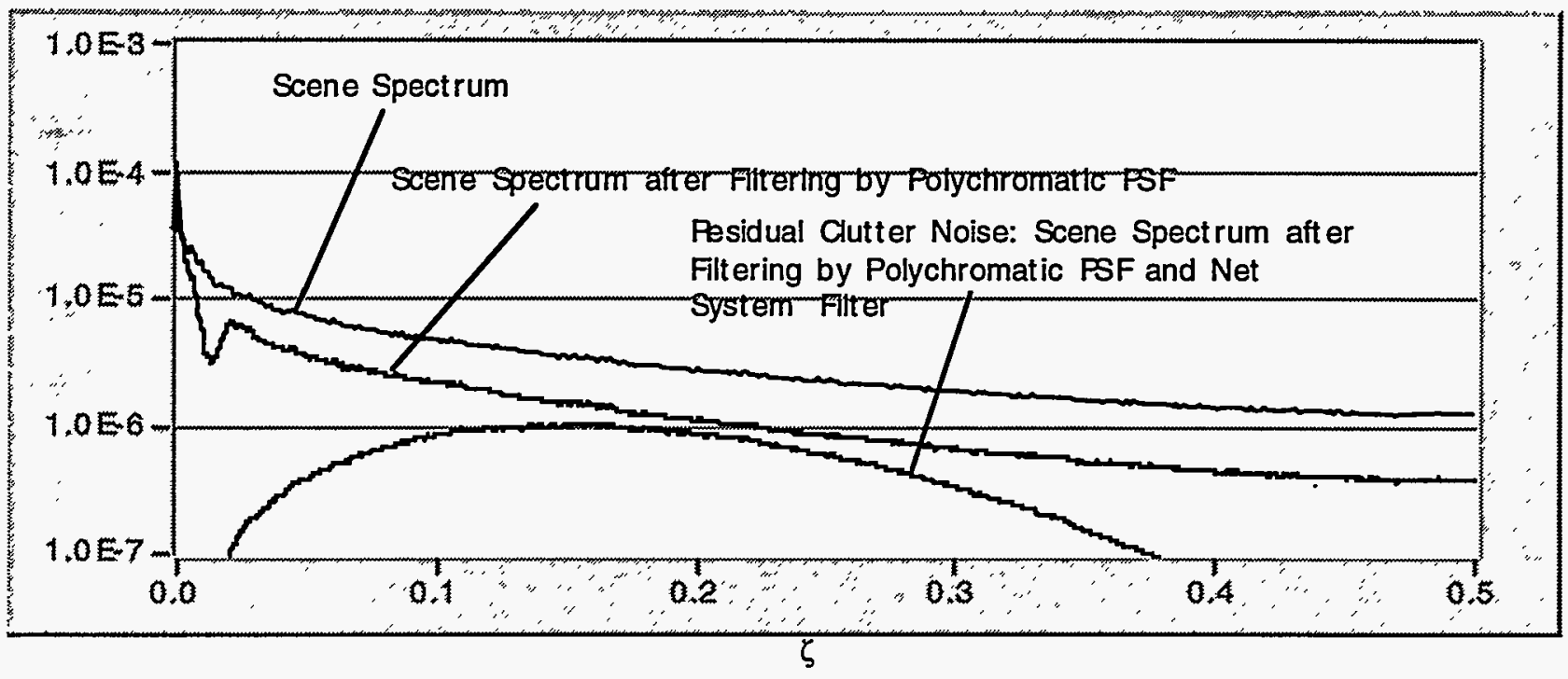

Figure 17: Residual RMS Clutter Noise Results 


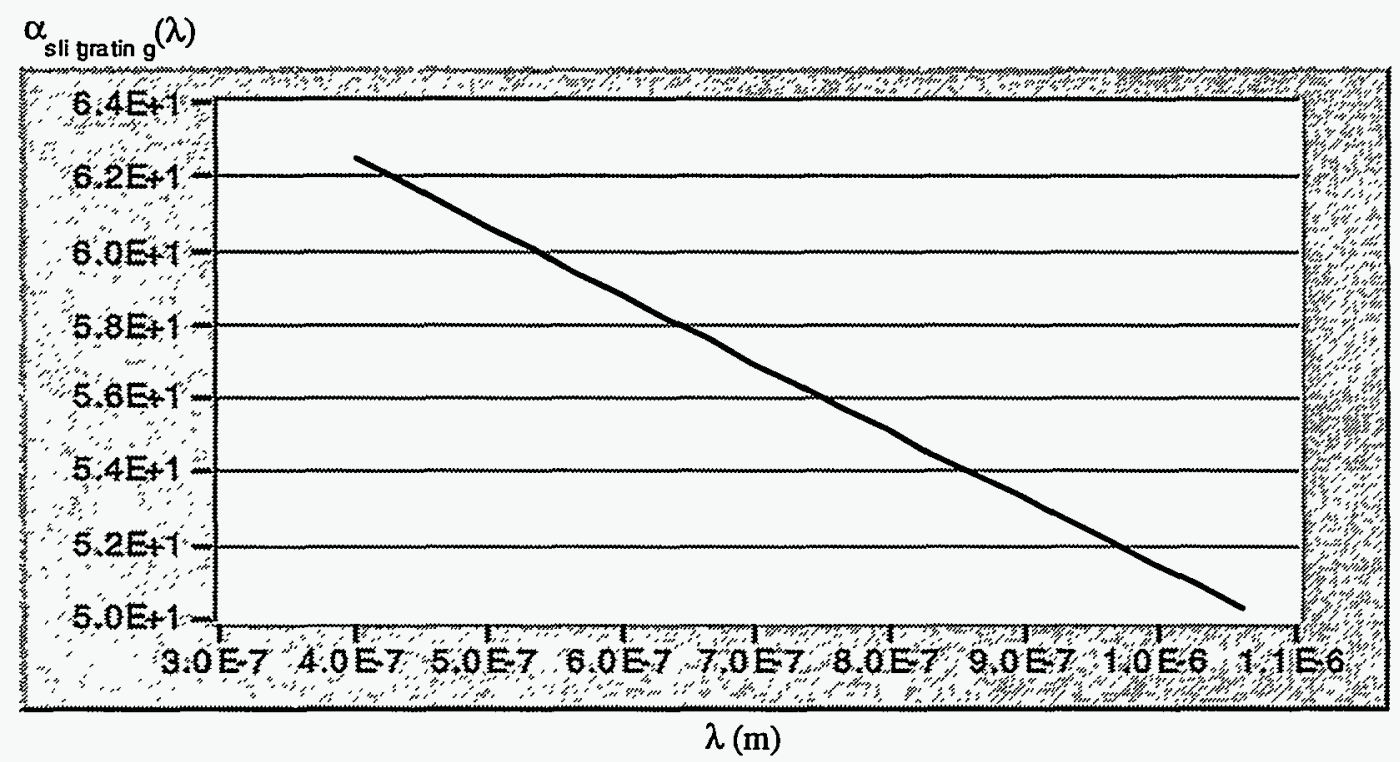

Figure 18: Variation of $\alpha$ with Wavelength for the Slit-Grating Design

The signal spectrum pictured in figure 14 shows that a significant percentage of the signal energy lies in regions of the frequency spectrum that are outside the net system-filter bandwidth. If a signal could be synthesized with a larger portion of its energy within this band-pass, the SNR would be directly improved. In addition, it is proposed that the resulting degradation in noise would be improved if such a function were found using the argument that signals with a tighter frequency spectrum will have a broader diffracted spatial profile. Thus the convolution of the scene with a broader diffraction PSF will yield a signal with lower overall noise in the higher frequencies where the signal detection occurs. Thus a proposed criteria for an optimum pupil function is that it have compact, band-limited signals that lie within the system-filter bandwidth, and have the highest percentage of its overall signal peaked at or near the peak of the overall system transfer function.

With this as a criteria for optimizing the SNR, a variety of optimum aperture function can be proposed. In the remainder of this report, three will be discussed: phase only functions, Young's double-slit and the Barker-coded double-slit.

\section{BRIEF DISCUSSION OF PHASE-ONLY PUPIL FUNCTIONS AS REPLACEMENTS FOR THE SLIT-GRATING DESIGN}

The slit-grating concept discussed above is a system that has a pupil that modulates the amplitude of the wave passing through it to produce the diffraction effect. Similar effects can be produced by modulating the phase of the wave as it passes through the aperture. A simple example of a phase aperture is a hologram which modulates the phase of a monochromatic wave to create the illusion of a reconstructed three-dimensional object.

Phase apertures were investigated for this application because the total energy in the diffraction pattern is higher in a system with only phase modulation in the aperture. Very little light is reflected or absorbed by the aperture. In the slit-grating concept, one-half of the light is lost due to the $50 \%$ 
duty cycle of the Ronchi grating function. With a phase aperture, virtually all the wave energy would be contained in the diffraction pattern, improving the SNR.

- The primary disadvantage for phase-only apertures is that the phase delay that a specific structure in the aperture would produce is inversely proportional to the signal wavelength. While it might be optimum for a single wavelength, the phase delay would be different for another wavelength. The resulting diffraction PSF from a given phase aperture will then dynamically change over wavelength. The diffraction PSF of phase apertures vary significantly over wavelength ranges less than a factor of two. Since the signal diffraction PSF is required to be a stable with wavelength, a phase aperture cannot be a valid pupil function for replacing the slit-grating concept. For this reason investigation into an optimum aperture was limited to amplitude modulation apertures only.

\section{APPLICATION OF LINEAR SYSTEM ANALYSIS TO YOUNG'S DOUBLE SLIT: A PROPOSED OPTIMUM APERTURE}

Since the scope of possible aperture functions was limited to amplitude-only apertures, the process of determining an optimum aperture is simplified significantly. The spatial frequency spectrum of amplitude-only apertures can be visualized directly by the autocorrelation of the amplitude transmission function of the aperture. Therefore if the optimum aperture was to be defined by the properties previously mentioned, it is a relatively simple task to specify one possible optimum aperture.

The natural function that is able to place energy into specific well-defined frequency bands is Young's double-slit. Diffraction in a Young's double-slit experiment yields a squared, sincmodulated cosine wave. As one would expect, the cosine wave has well defined frequency content which will yield a distinct peak in the spatial-frequency transform plane.

The form of the transmission function for the double-slit case is shown below in equation 76 . The double slit is defined as two perfectly transmitting regions of width $l_{\text {sli }-x}$ separated by a distance $a_{\text {slit_ } x}$.

$$
t_{1}(x)=r e c t\left(\frac{x-\frac{a_{\text {slit_x}}}{2}}{l_{\text {slit_x }}}\right)+\operatorname{rect}\left(\frac{x+\frac{a_{\text {slit_x }}}{2}}{l_{\text {slit_x }}}\right)
$$

With the assumptions given previously that $\left|A B_{l}\right|^{2}$ is one, the diffraction pattern on the focal plane can be written using the Fourier-transformation relationship given in equation 13. 


$$
\begin{aligned}
& I_{\text {laser }}(x, y, \lambda)=\frac{1}{(\lambda f)^{2}}\left|F F\left[\operatorname{rect}\left(\frac{x-\frac{a_{\text {slit_ } x}}{2}}{l_{\text {slit } x}}\right)+\operatorname{rect}\left(\frac{x+\frac{a_{\text {slit }} x}{2}}{l_{\text {slit } x}}\right)\right]\right|_{\xi=x / \lambda f}^{2} \\
& =\frac{1}{(\lambda f)^{2}}\left|F F\left[\operatorname{rect}\left(\frac{x}{l_{s l i t_{-} x}}\right) * \frac{2}{a_{s l i t_{-} x}} \delta \delta\left(\frac{2 x}{a_{s l i t_{-} x}}\right)\right]\right|_{\xi=x / \lambda f}^{2} \\
& =\left(\frac{2 l_{\text {slit }-x}}{\lambda f}\right)^{2}\left|\sin c\left(\frac{l_{\text {slit_- }} x}{\lambda f}\right) \cdot \cos \left(\pi \frac{a_{\text {slit_x }} x}{\lambda f}\right)\right|^{2}
\end{aligned}
$$

As expected, the monochromatic diffraction signal due to the double slit is a squared, sincmodulated cosine wave. The more important representation of the signal for this report is the spatial frequency representation of the signal energy which can be determined from equation 20 where the signal spatial frequency spectrum can be determined from the autocorrelation of the aperture transmission function.

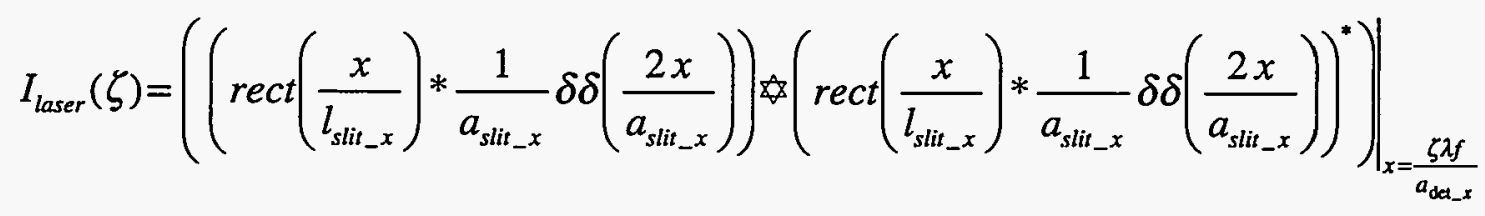

Since all the functions are bilaterally symmetric, the autocorrelation and convolution operations are equivalent. The spatial frequency spectrum for the double-slit modulated laser signal can be written is closed form.

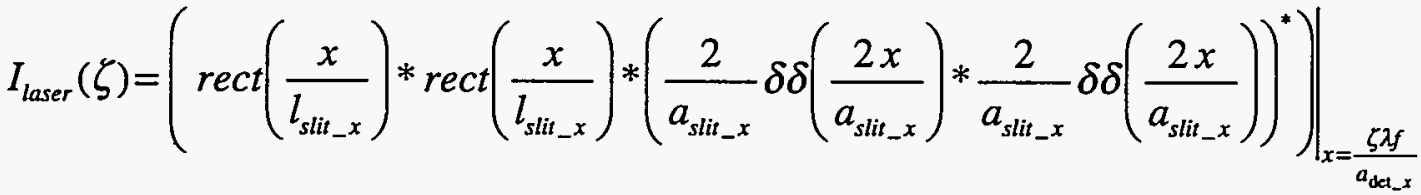

$$
\begin{aligned}
& =\left.\left(l_{s l i t_{-} x} \cdot \operatorname{tri}\left(\frac{x}{l_{\text {slit } x}}\right) *\left(\frac{1}{a_{\text {slit } x}} \delta \delta\left(\frac{x}{a_{\text {slit } x}}\right)+2 \delta(x)\right)^{*}\right)\right|_{x=\frac{\zeta \lambda f}{a_{\text {det_ } \_}}} \\
& =2 l_{\text {slit }-x} \cdot \operatorname{tri}\left(\frac{\zeta \lambda f}{l_{\text {slit_x }} a_{\text {det }_{-} x}}\right) *\left(\frac{1}{2 a_{\text {slit_x }}} \delta \delta\left(\frac{\zeta \lambda f}{a_{\text {slit_x } x} a_{\text {det }_{-} x}}\right)+\delta\left(\frac{\zeta \lambda f}{a_{\text {det }_{-} x}}\right)\right)
\end{aligned}
$$

This spectrum, using example values to be determined below, is shown in figure 26 . By examining the above relationship, the laser signal spectrum is seen to have three peaks, one at DC and a pair at

$$
\zeta_{0}= \pm \frac{a_{\text {slit_x } x} a_{\mathrm{det}_{-} x}}{\lambda f} .
$$


This pair represents the squared cosine fundamental of the laser signal. By adjusting $a_{\text {slit }} x$ the modulation frequency at the mean laser wavelength can be adjusted to lie directly under the peak of the system transfer function to optimize the signal throughput over the laser bandwidth required. Using a Si photodiode response as a baseline, $0.4 \mathrm{um}-1.1 \mathrm{um}$, the mean wavelength is $0.75 \mathrm{um}$. Since the peak in the system transfer function occurs at around $\zeta=0.21$, an optimum slit spacing can be computed to be approximately $60 \mathrm{um}$.

$$
a_{\text {slit } \_x}=\frac{\zeta_{0} \lambda_{\text {mean }} f}{a_{\text {det } \_x}}=\frac{0.21 \cdot 0.75 \mu \mathrm{m} \cdot 11.4 \mathrm{~mm}}{30 \mu \mathrm{m}}=60 \mu \mathrm{m}
$$

The width of the slits adjusts the spatial frequency band around the fundamental that the signal occupies. To maximize the total power through the slits by maximizing slit width, while maintaining a distinct minimum between the dc component and the cosine modulated signals, the optimum width of the slit is seen to be one half the slit spacing.

$$
l_{\text {slit_x }}=\frac{a_{\text {slit_x }}}{2}=30 \mu m
$$

Before accepting these parameters as the optimum, some examination is necessary into the signal spectra produced by this aperture as a function of frequency, as it pertains to wavelength determination. In the slit-grating system the wavelength would be determined from the position of the first-order peak relative to the zero-order peak in the spatial domain. If one were to use a double-slit aperture system to determine wavelength, the natural domain to look for peaks is in the spatial-frequency domain, where the peak representing the fundamental modulation frequency of the signal varies as a function of laser wavelength. If wavelength determination were a requirement, then there would have to be well-defined peaks in the spatial frequency domain for every possible laser signal.

The frequency spectra resulting from the double slit case above been illustrated in figure 19. It is seen that the residual signal from the dc component begins to have a peak that exceeds the first order fundamental peak at the short end of the wavelength spectrum around $0.4 \mathrm{um}$. If one were trying to determine wavelength in this case, a wrong determination would be made off the residual $\mathrm{dc}$ term. In addition, the system transfer function blurs the peak in the first-order spectrum making it hard to determine a well defined peak anywhere below $0.5 \mathrm{um}$. It is also seen that this aperture design violates one of the design rules stated above, that the signal spectra be contained within the pass-band of the system over all wavelengths.

A second cut at the double-slit aperture design requires moving the slits closer together, thereby reducing the fundamental modulation frequency of the laser signal on the detector. The requirement used to develop the new slit spacing is to ensure that the maximum spatial frequency content of the shortest wavelength is less than the Nyquist frequency for the system. Solving equation 79 to achieve this at 0.4 um yields,

$$
\begin{aligned}
& a_{\text {slit_x }}=50 \mu \mathrm{m} \\
& l_{\text {slit_x }}=25 \mu \mathrm{m},
\end{aligned}
$$



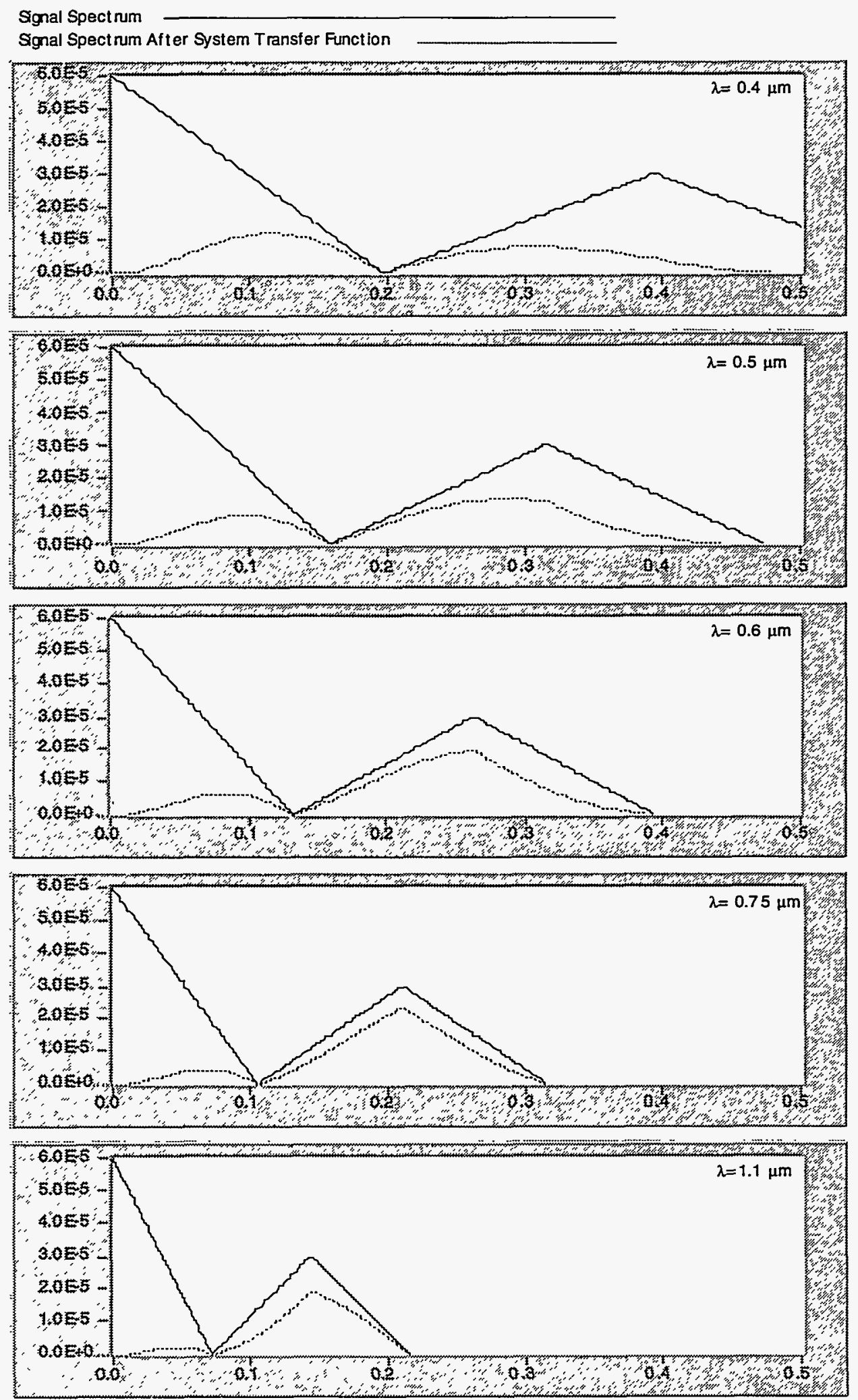

Figure 19: Laser Signal Frequency Spectra for 60 um Slit Spacing 
Signal Spectrum

Signal Sp ectrum Aft er System Transfer Function
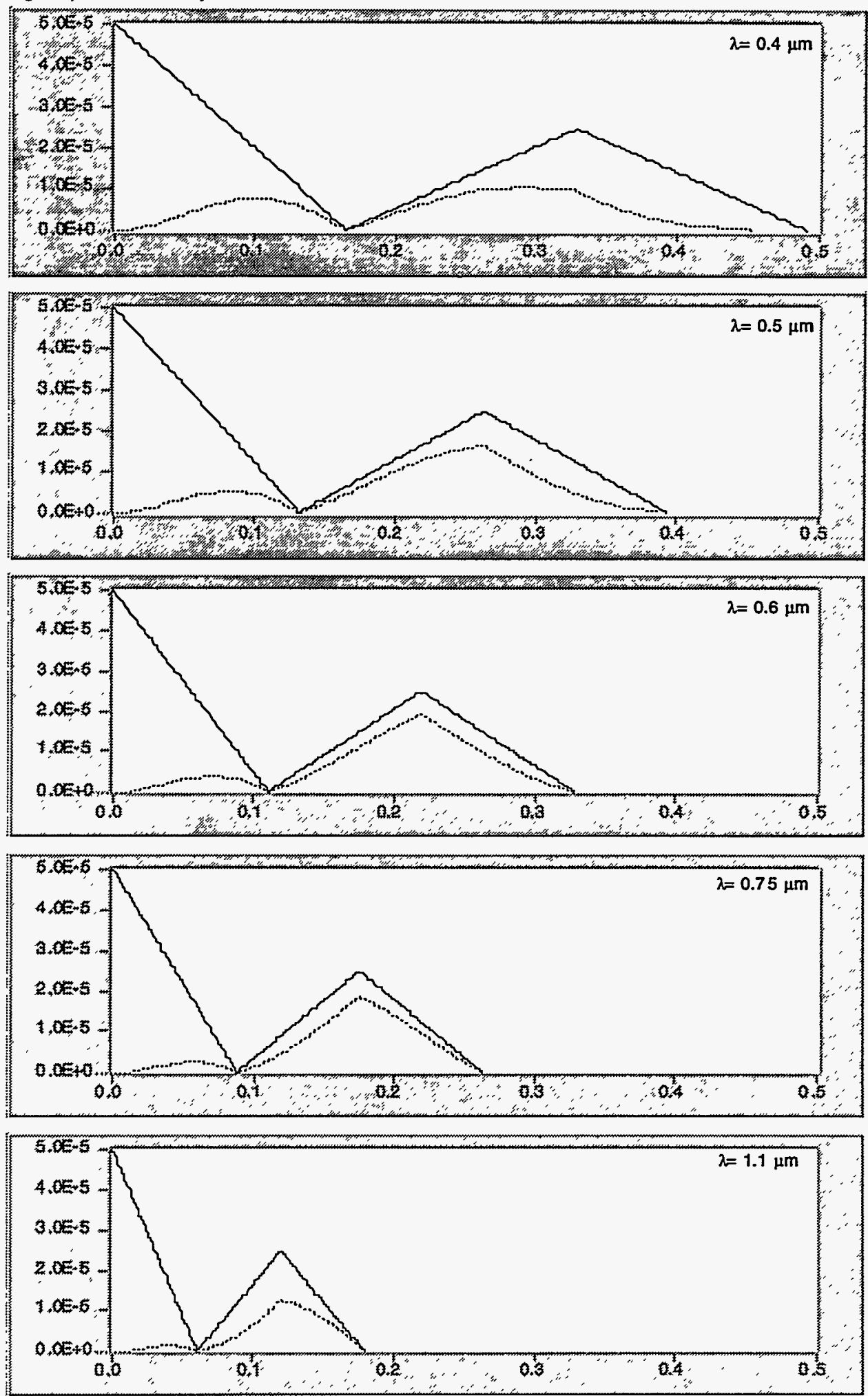

Figure 20: Laser Signal Frequency Spectra for 50 um Slit Spacing 
Laser Signaly


Laser Signal After Systëm Transfer Function
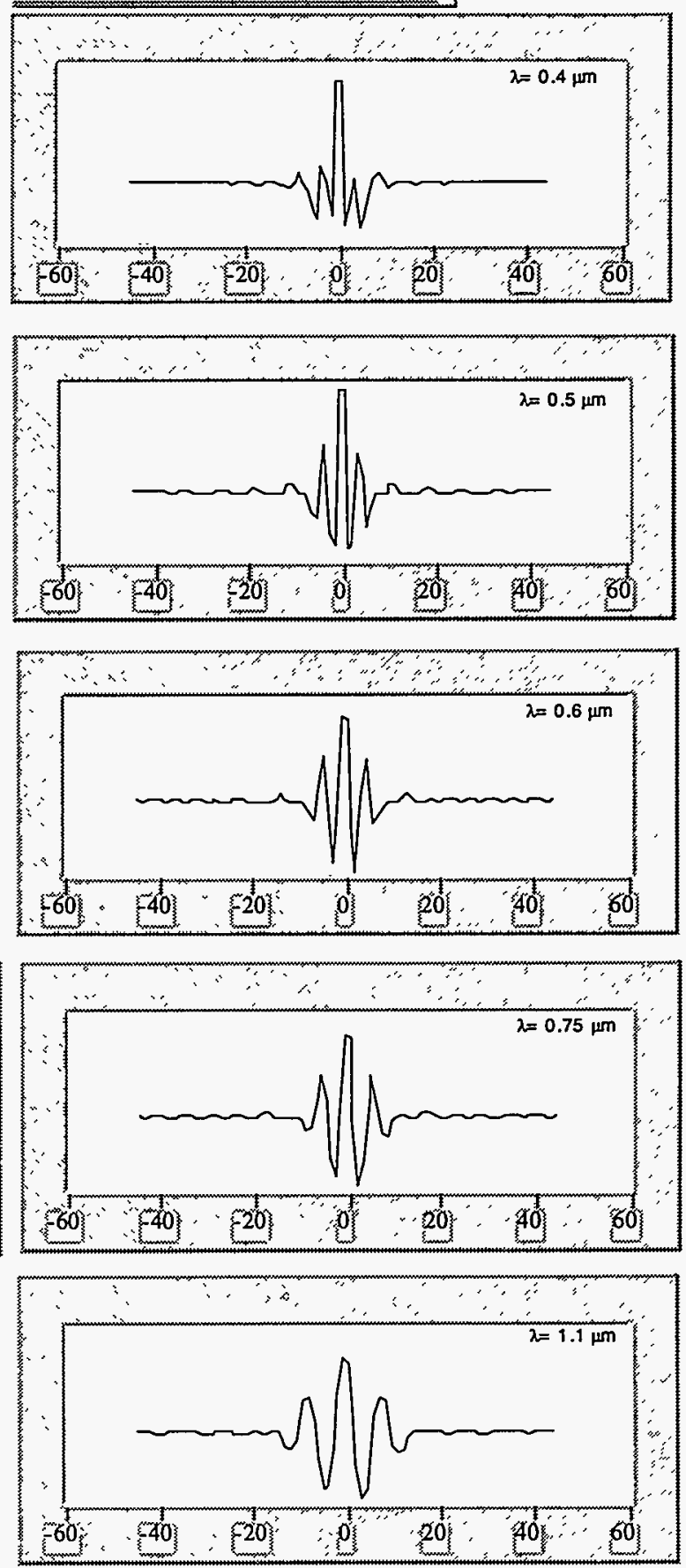

Figure 21: Sample-Space Laser Signal Profiles vs. Wavelength for 50 um Slit Spacing 
assuming a distinct minimum is required in the spectra between the low-frequency band and the fundamental. The resulting signal spectra for this design are shown in figure 20. It is seen that one has regained a moderately distinct spectral peak at the $0.4 \mathrm{um}$ as required, and a distinct peak by $0.5 \mathrm{um}$. The laser signals from these spectra are shown in figure 21 before and after filtering by the system. It is evident that the distortion at short wavelengths is present, but since the determination of laser wavelength would be done in the frequency domain, this distortion might not effect the detection mechanism. If it were found that this distortion were unacceptable, then the slit spacing could be further decreased to relax the distortion at the expense of system throughput. Using the same technique as in the slit-grating case discussed in a previous section, the polychromatic diffraction PSF was computed for the same silicon linear array. The diffraction PSF is shown below in figure 22 as well as its corresponding filter function in figure 23.

As in the slit-grating design discussed previously, the rms clutter-noise is found according to the denominator of equation 51 . Shown below in figure 23 is the spatial-frequency spectrum of the clutter noise in the double-slit design after application of the system transfer function and the polychromatic PSF related filter function. While it may appear that the clutter in the double-slit design is lower than in the slit-grating design, the real comparison must be made in the improvement in SNR which is gauged by the figure of merit $\alpha$.

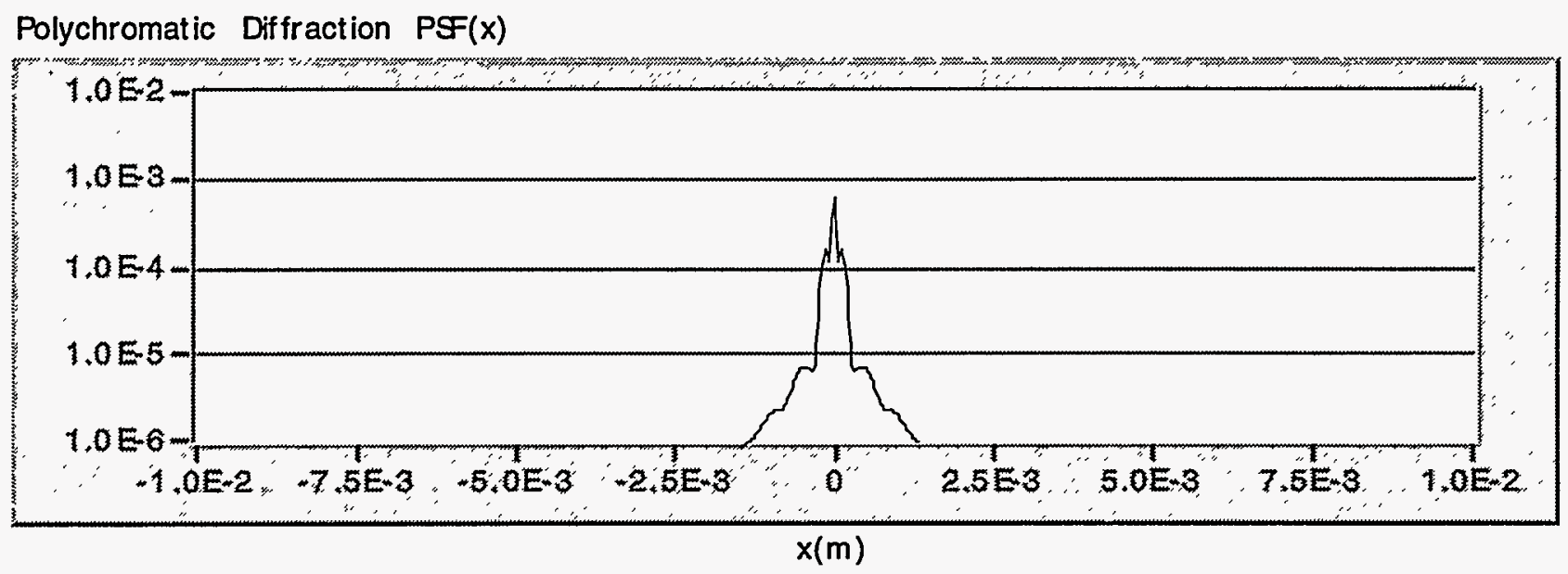

Figure 22: Polychromatic Diffraction PSF for the 50 um Double-Slit Design

The figure of merit $\alpha$ was computed and is shown in figure 24 below. Since $\alpha$ is a measure of the RMS SNR, this plot shows that SNR peaks around 0.5 um due to the interplay of the signal spectrum as a function of wavelength and the system transfer function. This differs from the slitgrating case where the signal energy was a monotonically decreasing function of frequency so the amount of energy under the transfer function varied directly with wavelength. 


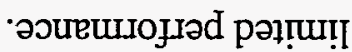

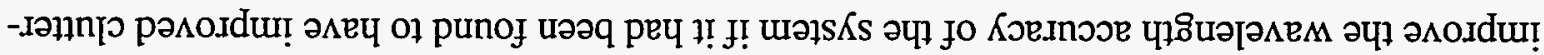



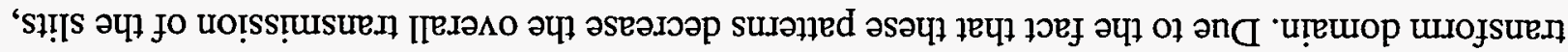

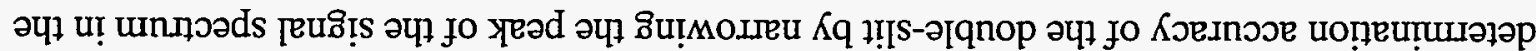

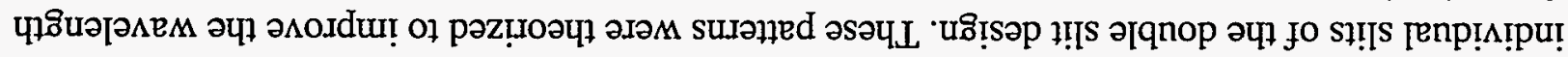

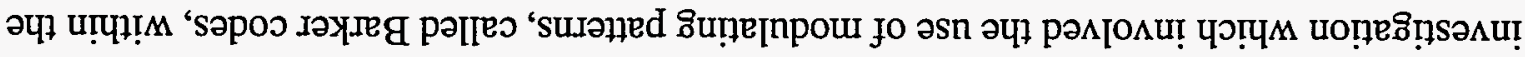

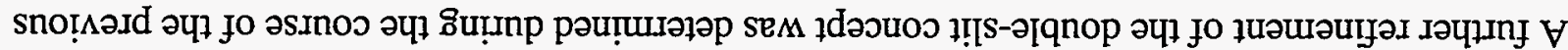

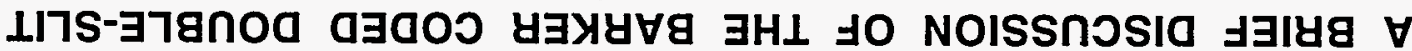 \\ IdヨJNOO}

'pəsn әIәм suọ̣e[nuuroj

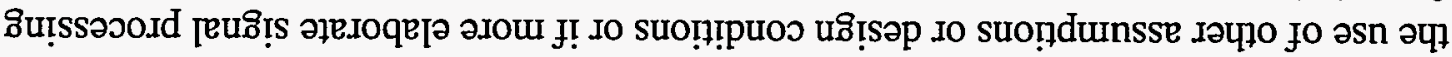

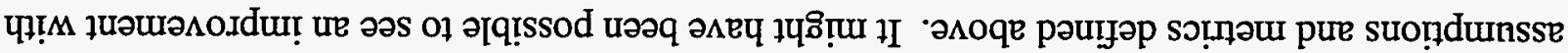

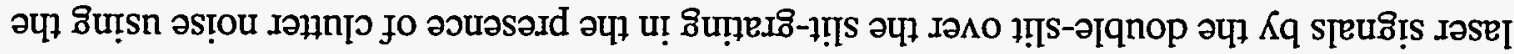

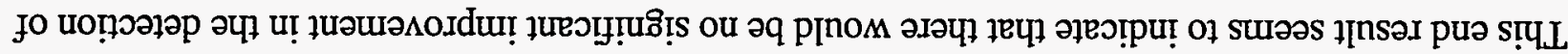

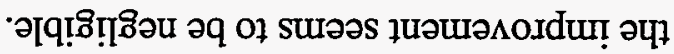

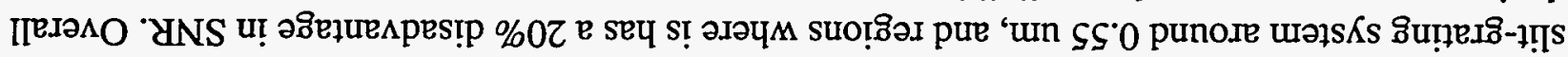

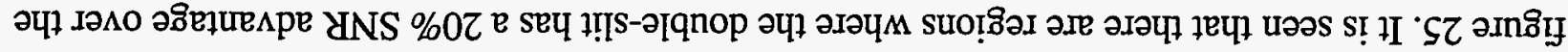

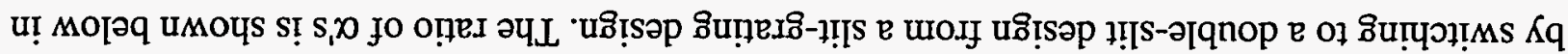

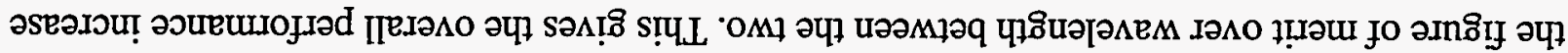

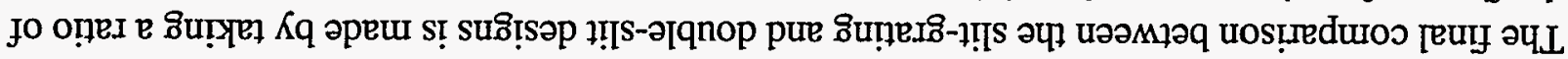

SNDISヨa

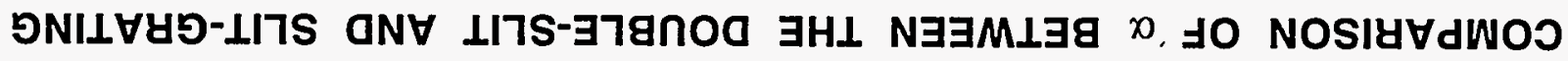

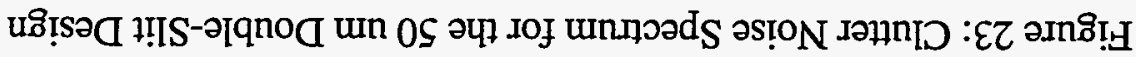

5

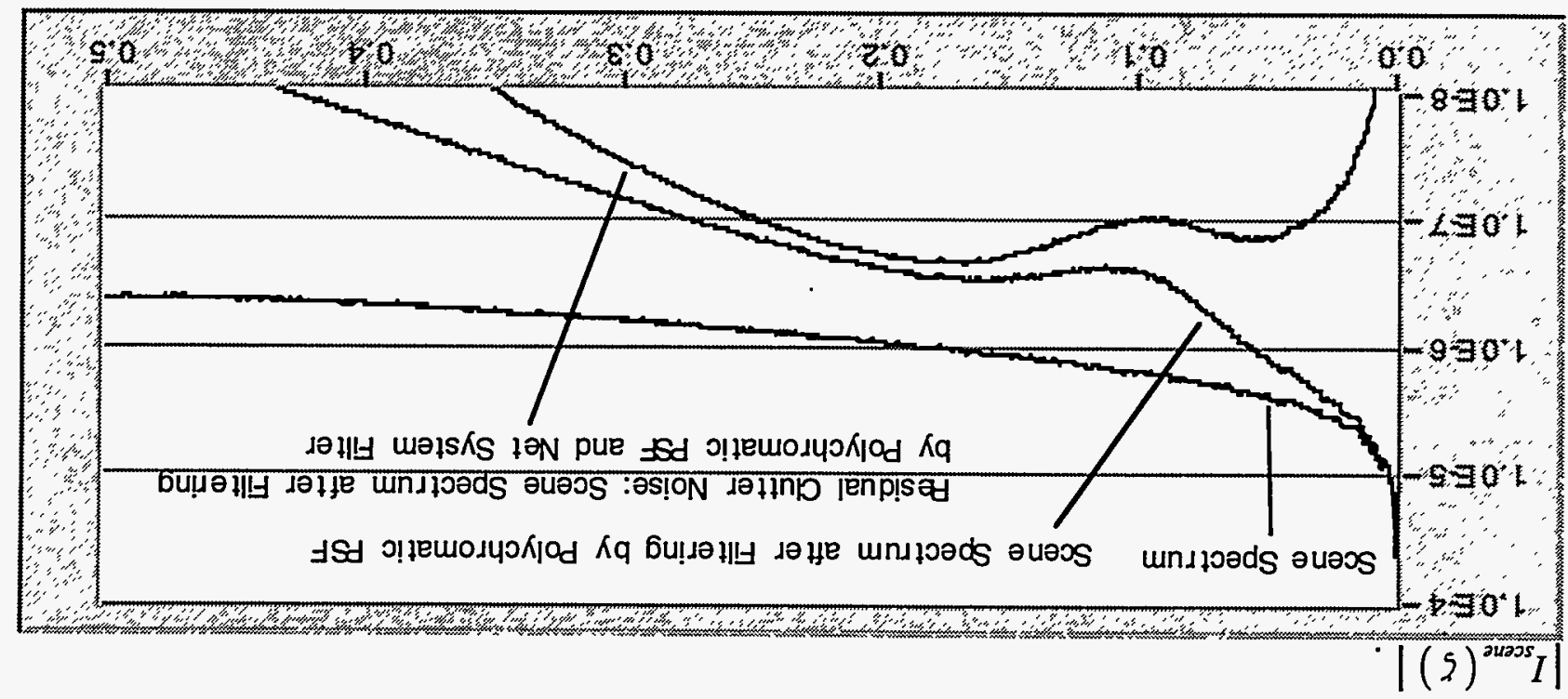




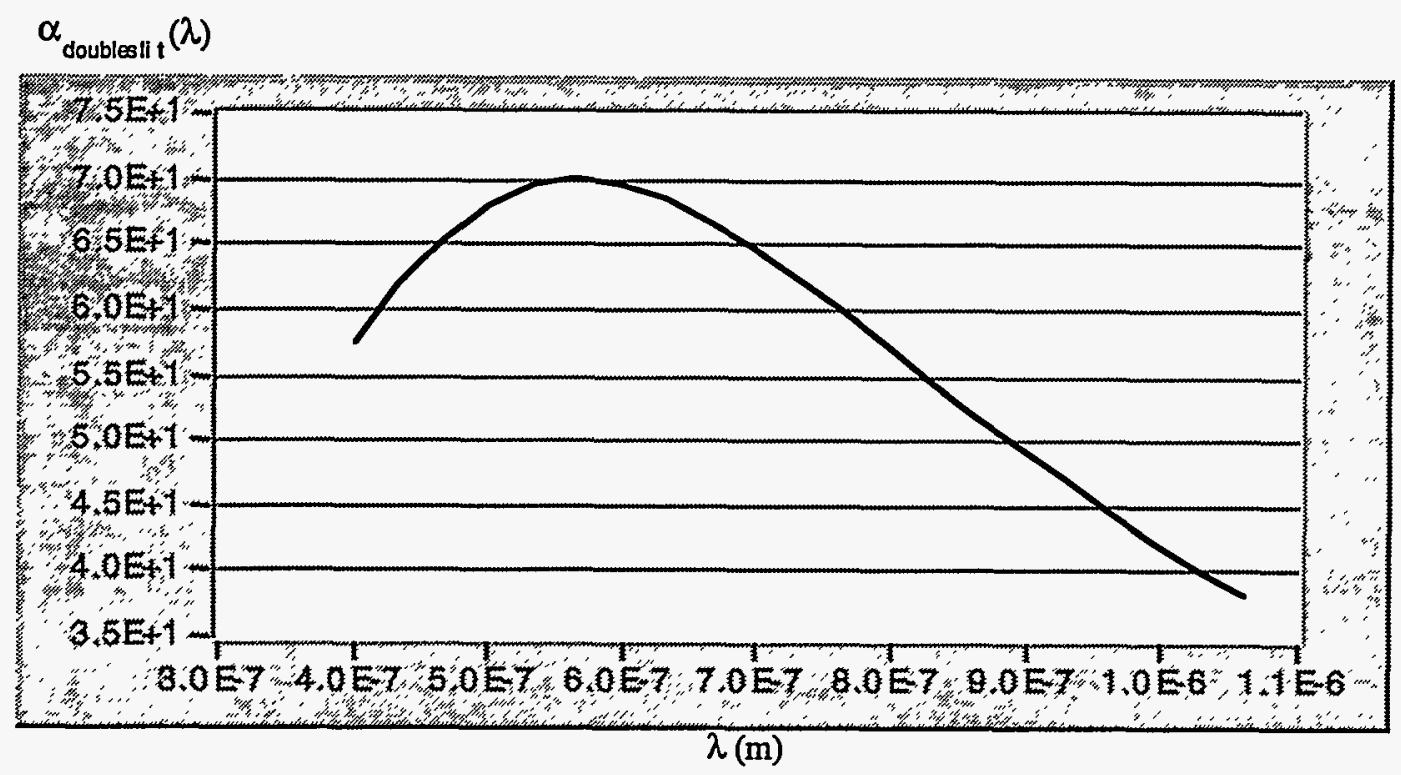

Figure 24: Variation of $\alpha$ with Wavelength for the Double-Slit Design

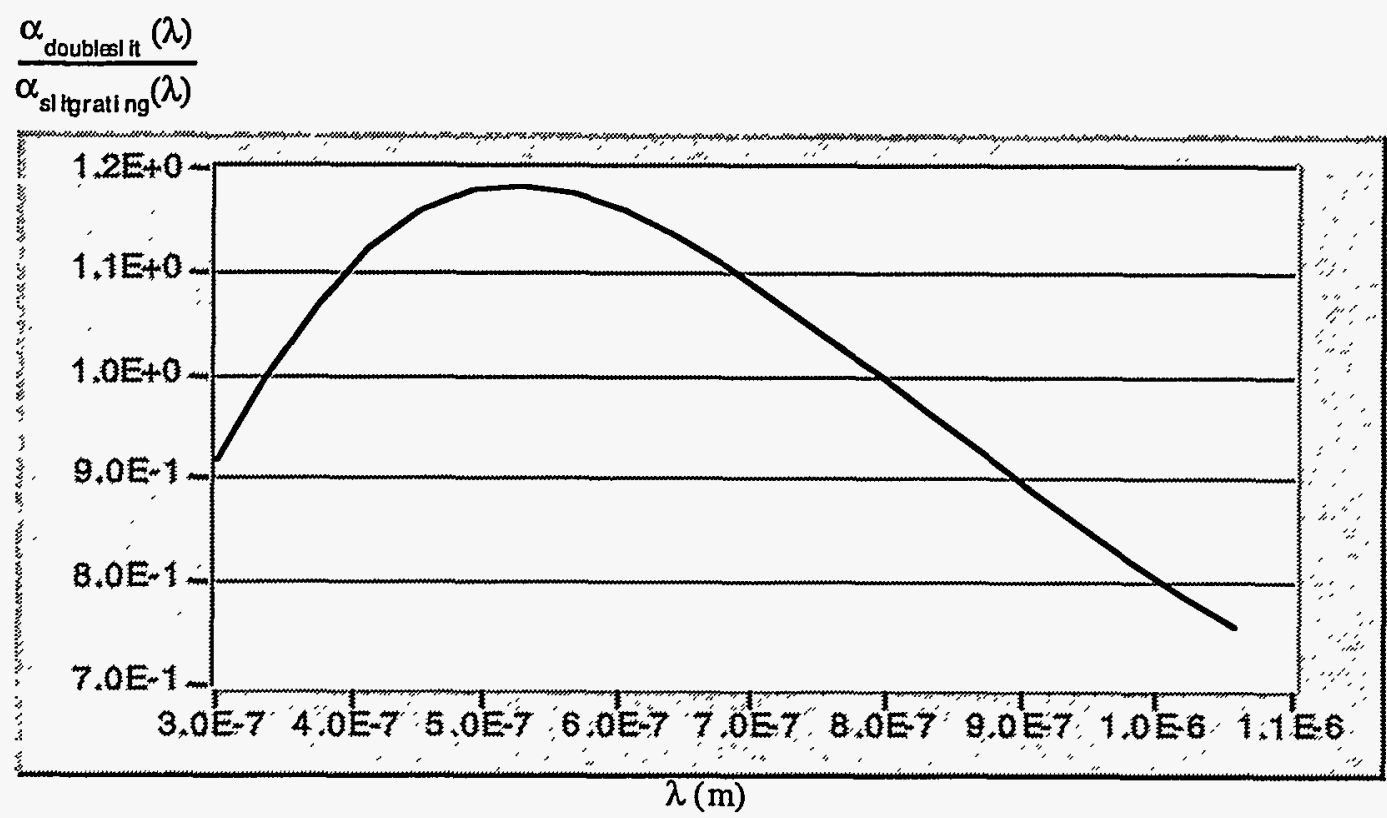

Figure 25: SNR Improvement of the Double-Slit Design Over the Slit-Grating Design 
Barker codes, which are used to frame synchronize pulse-code-modulated telemetry systems, were designed to have strong autocorrelation properties. Since the spatial frequency spectrum of the laser signal is directly related to the autocorrelation of the pupil function, it was theorized that these patterns could be used to generate diffractive patterns that possessed a narrower peak in the signal spectra than the plain double-slit concept. If wavelength determination were made in the frequency domain, this would significantly improve the wavelength accuracy of the system because the peaks in the spectra would narrower.

According to the theory discussed above, the only way to narrow the peak in the signal spectrum for the double-slit case was to narrow the width of the slit. While this would accomplish the goal of better wavelength accuracy, the overall throughput of the aperture would directly decrease. The Barker-coded apertures presented a compromise, better wavelength resolution with only moderate compromise in aperture throughput. The binary Barker codes used are shown below in table 1.

Coded double-slit apertures were designed using these patterns using the same design methodology used with the double-slit concept above. The results of these designs are shown in appendix A. The width of the pattern shown in the appendix represents the total width of the code including trailing zeros. To achieve the desired zero in the signal spectrum between the low and high-frequency bands as was done in the double-slit case, the total width of the code is scaled to the value shown.

By examining the general properties of the resulting signal spectra, it is seen that the width of the fundamental peak becomes increasingly narrower as predicted. One can see that as various sources of spectral blurring would be introduced, the patterns with higher number of bits would preserve the accuracy of the peak position better than the lower numbered bits patterns, as well as the plain double-slit pattern.

This accuracy is achieved at the cost of the throughput of the aperture. The integrated width of each pattern, which is directly proportional to the dc and fundamental peak amplitudes in the signal spectra, is given with each design in the appendix. The integrated width of the patterns as a function of the number of bits in the pattern is plotted below in figure 26. The double-slit is represented as a code length of one. One can see that the peak signal in the frequency domain is immediately degraded by $20 \%$ from the double-slit case by the application of the first seven bit code. Since this decrease in aperture effects both signal and background, the net figure of merit used to evaluate the double-slit case would not significantly change for the coded aperture as long as the assumption of clutter-limited detection was still valid. 
Peak Signal in Frequency Domain [Integrated Pattern Width (m)]



Figure 26: Peak Signal vs. Barker Code Length

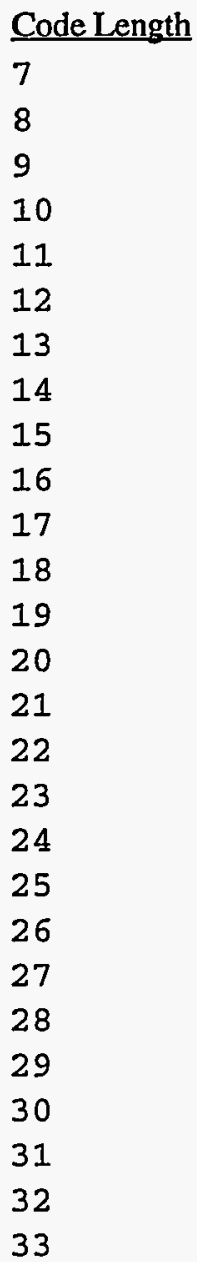

Code Length

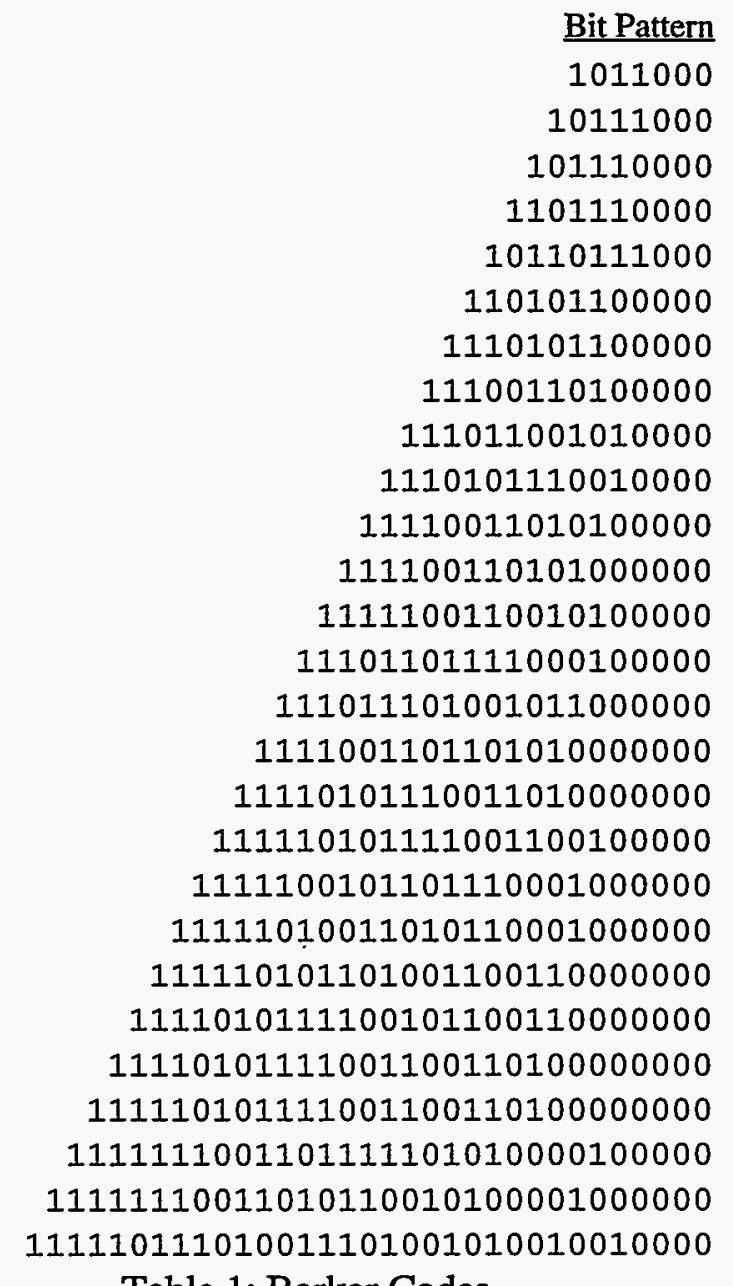

Table 1: Barker Codes 
If the double-slit concept had shown increased clutter limited performance, the Barker coded aperture could have been implemented as a method of improving the wavelength accuracy of the design. In addition, there might have been signal processing techniques that could take advantage of the structure of the signal spectrum to improve the clutter limited detection performance.

\section{CONCLUSIONS}

This investigation into methods of optimizing the clutter-limited detection performance of the slitgrating concept by changing the aperture transmission function showed that the double-slit approach did not significantly improve detection capability based on an RMS signal-to-noise merit function. In addition, phase functions were determined to have too much chromatic variation to justify the added benefit of increased aperture. Where the double-slit approach might be usefull, Barker coded double-slits were proposed as a novel addition to the double-slit approach which would improve the wavelength resolution if the wavelength determination were made in the spatial frequency domain.

In addition to the primary results stated above, the analysis of the entire electro-optical system used in this report, provides a good general approach that can be used as a template for reapplication in other sensor design studies. The simple techniques would be easily reapplied to the entire collection, detection and processing chain of an imaging optical sensor system. 


\section{APPENDIX A: BARKER CODED DOUBLE SLIT DESIGN RESULTS}

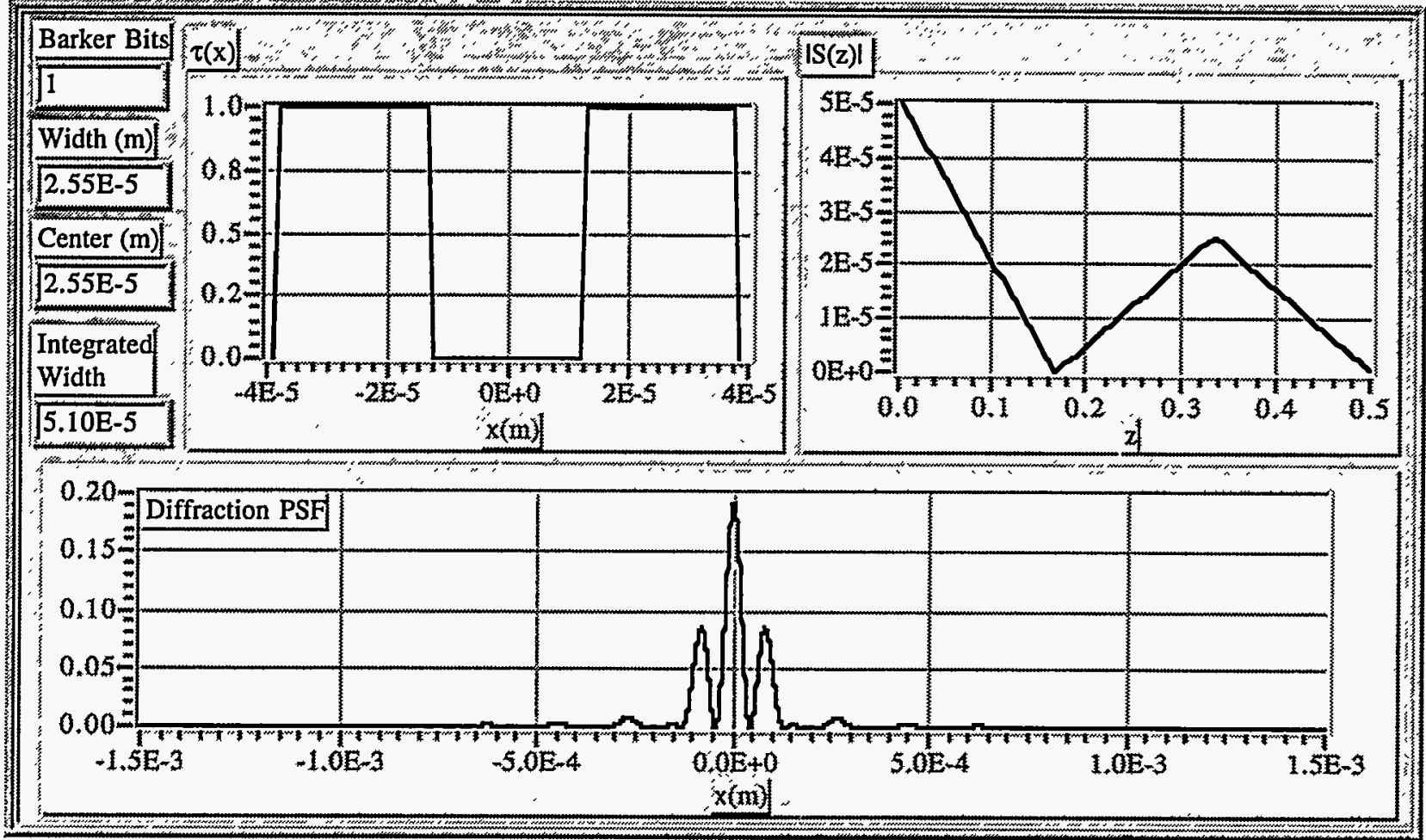

Figure A1: Young's Double Slit (1 Bit Barker Code)

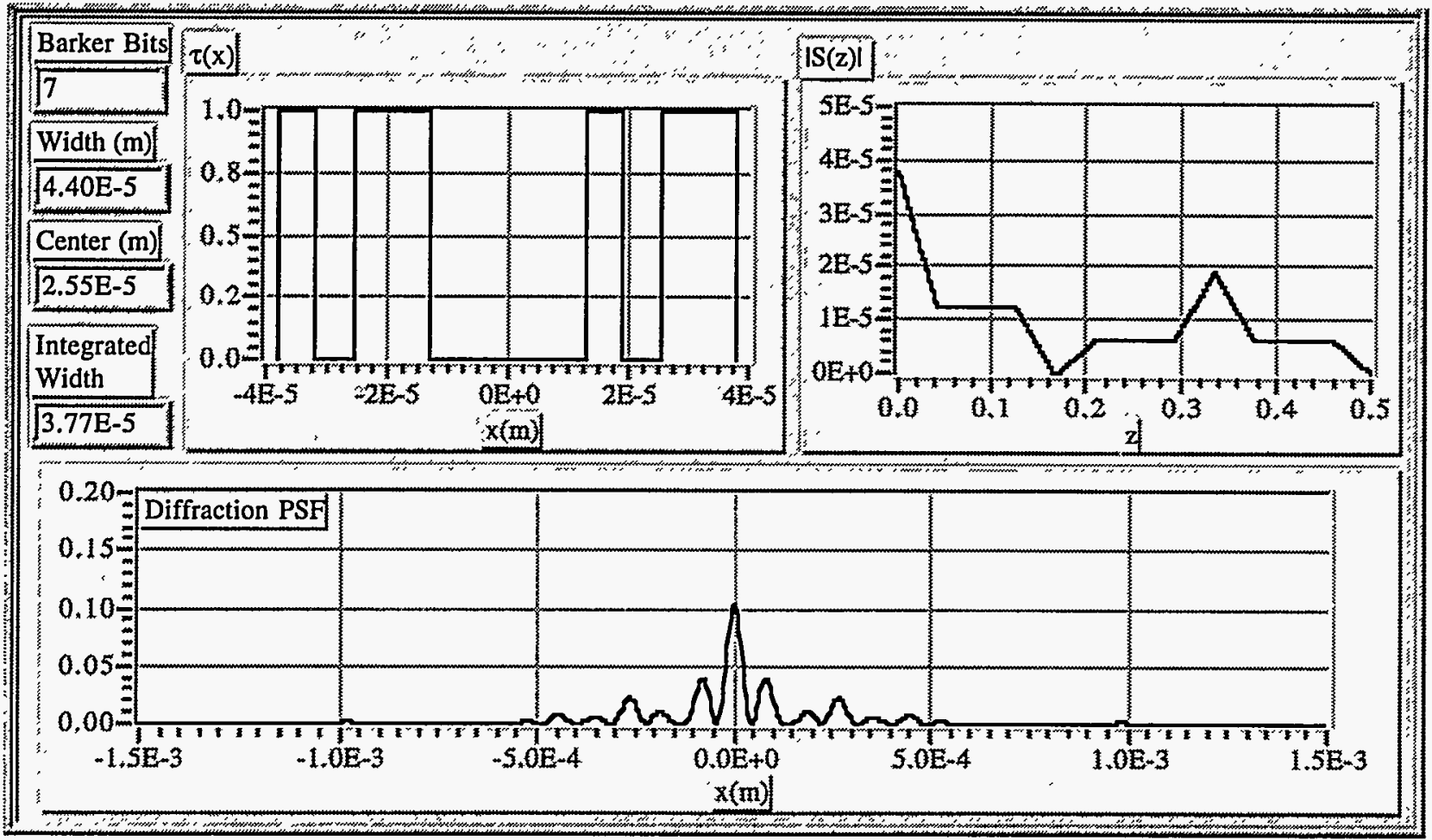

Figure A2: 7 Bit Barker Code Results 


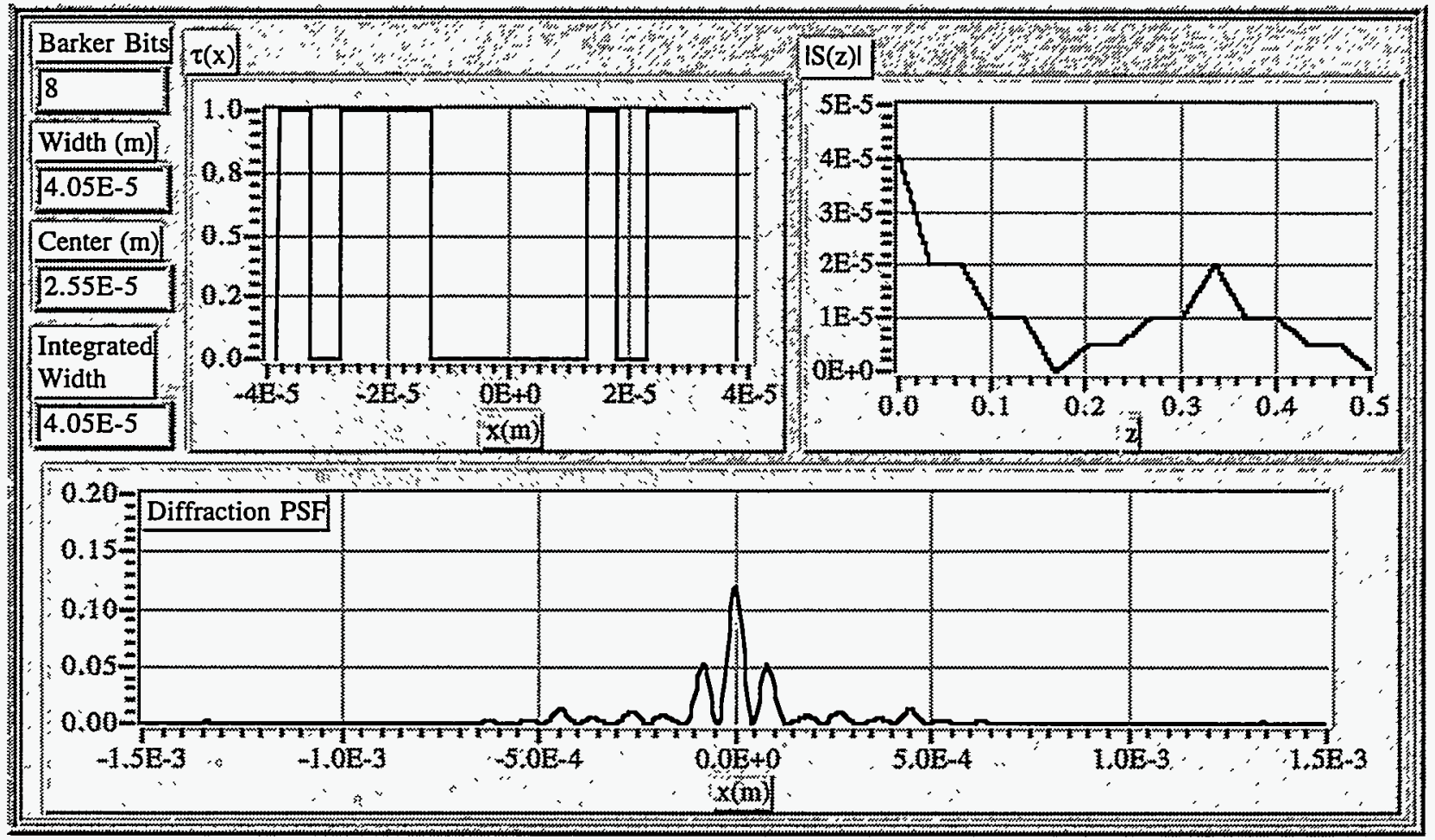

Figure A3: 8 Bit Barker Code Results

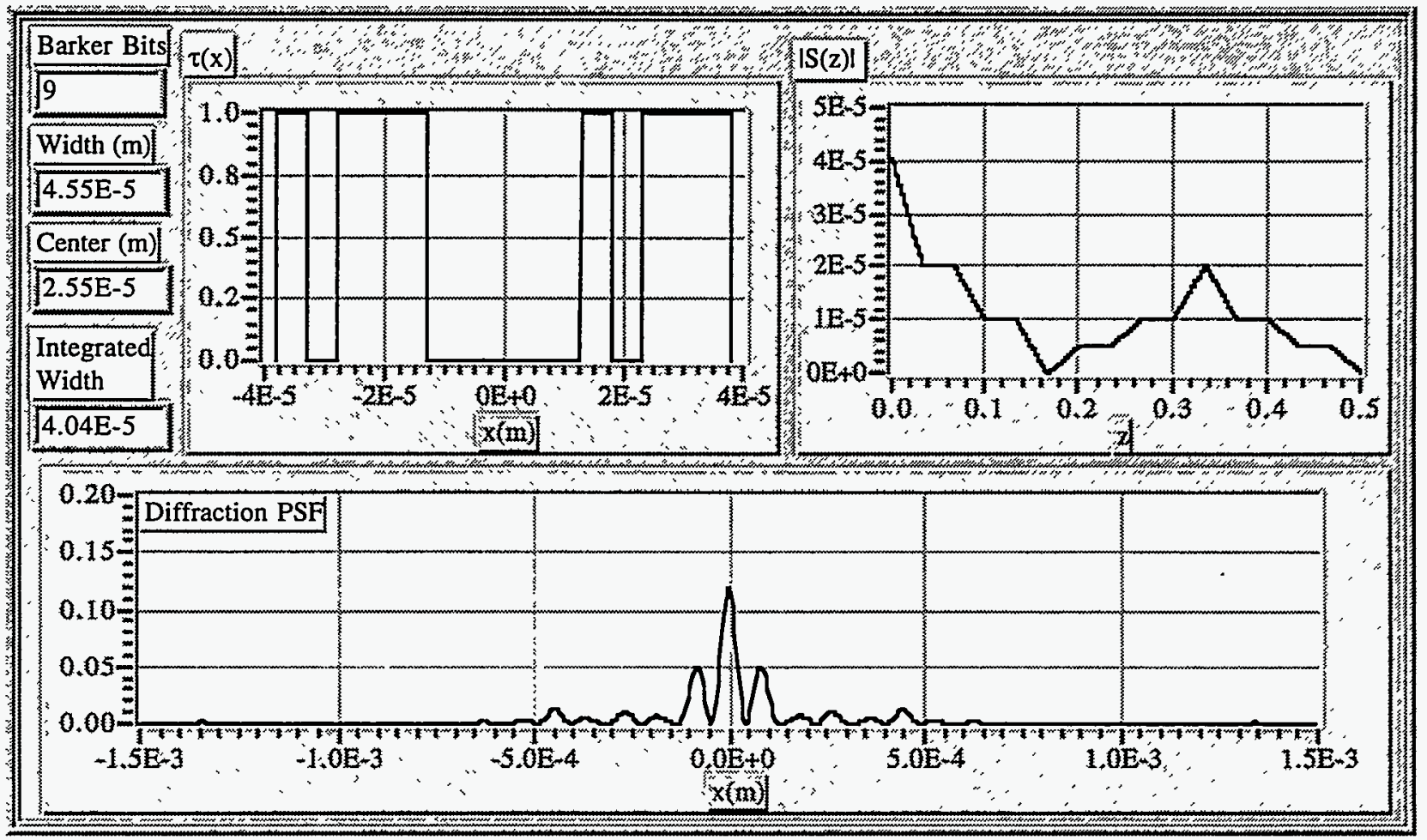

Figure A4: 9 Bit Barker Code Results 




Figure A5: 10 Bit Barker Code Results

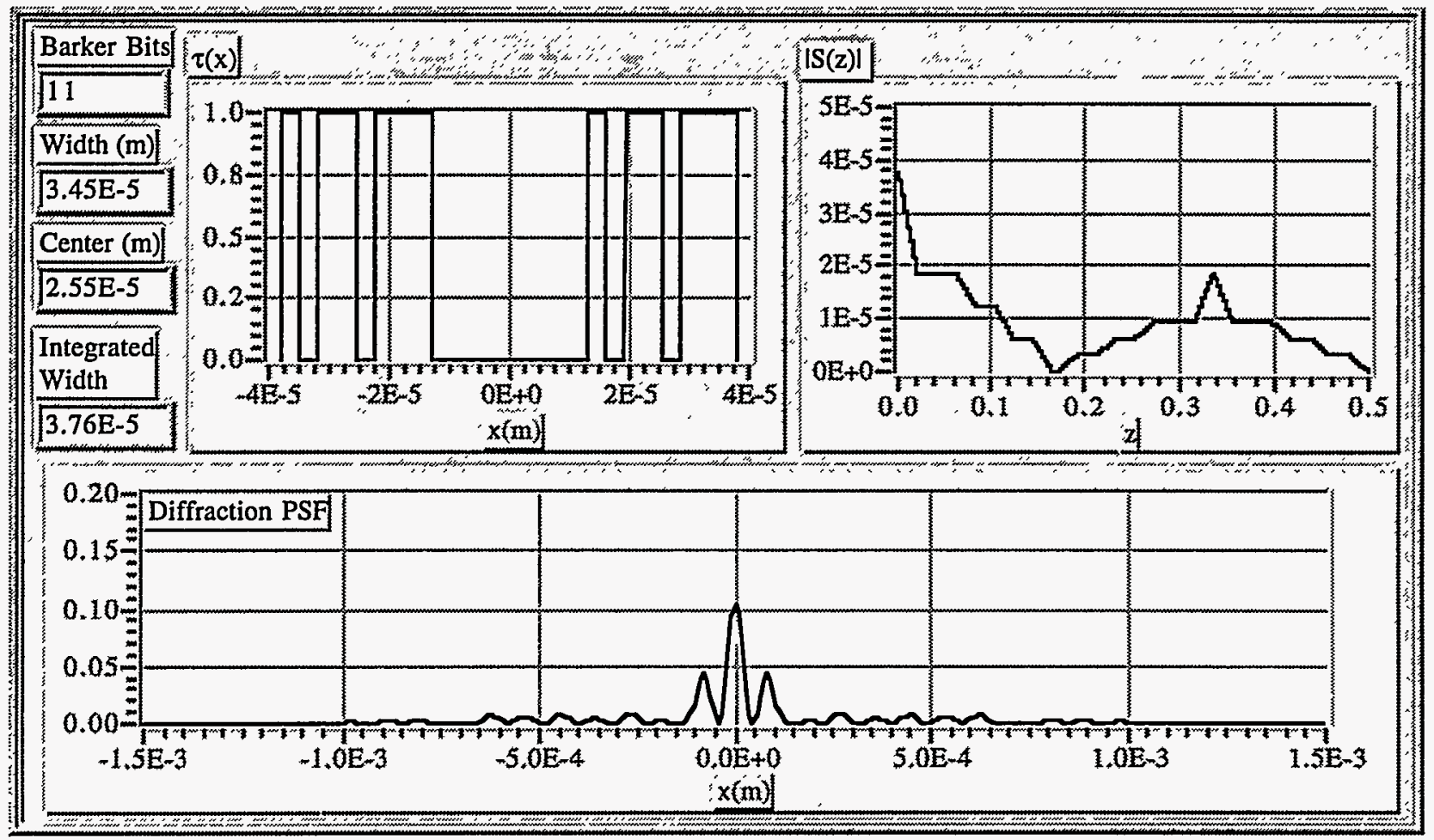

Figure A6: 11 Bit Barker Code Results 


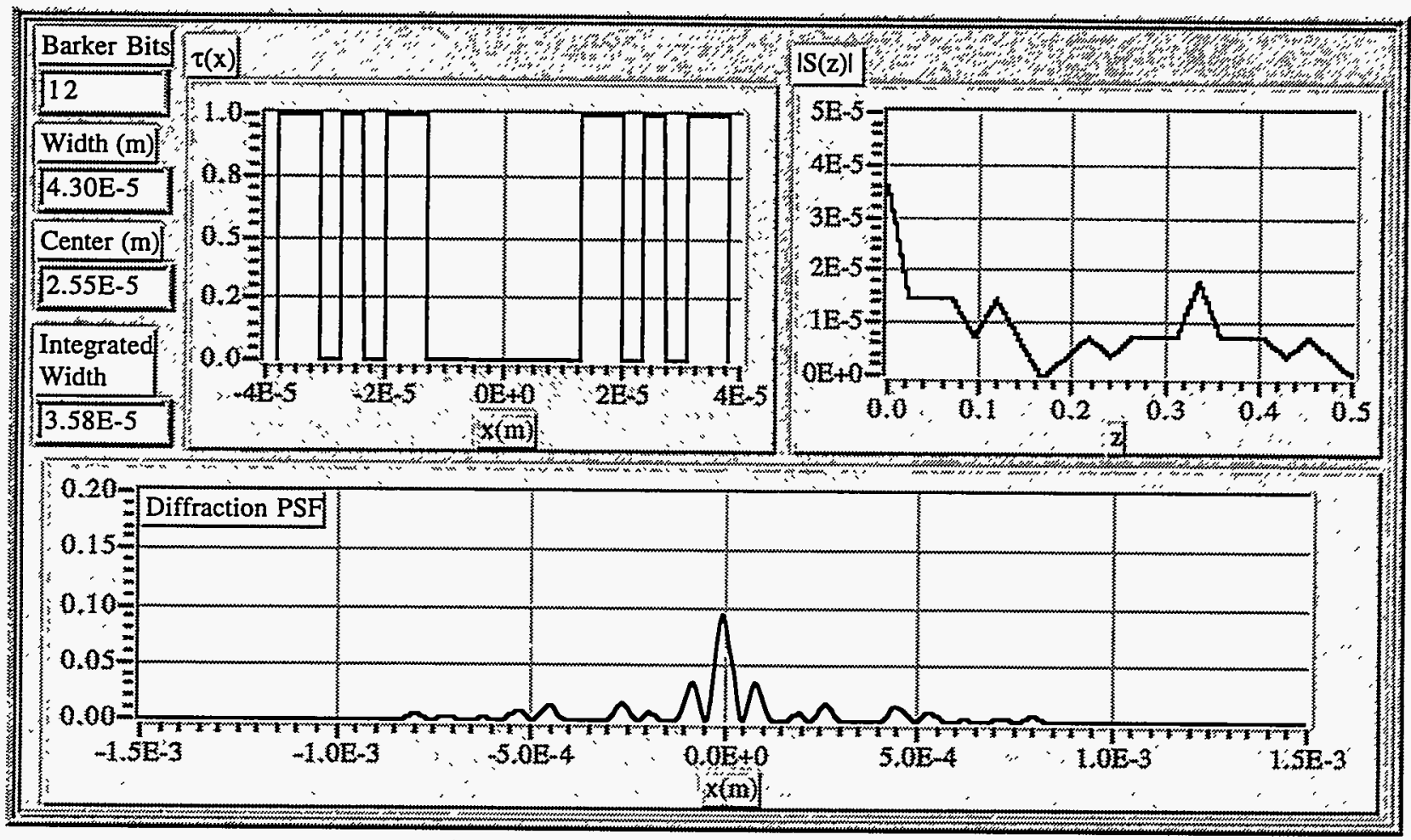

Figure A7: 12 Bit Barker Code Results

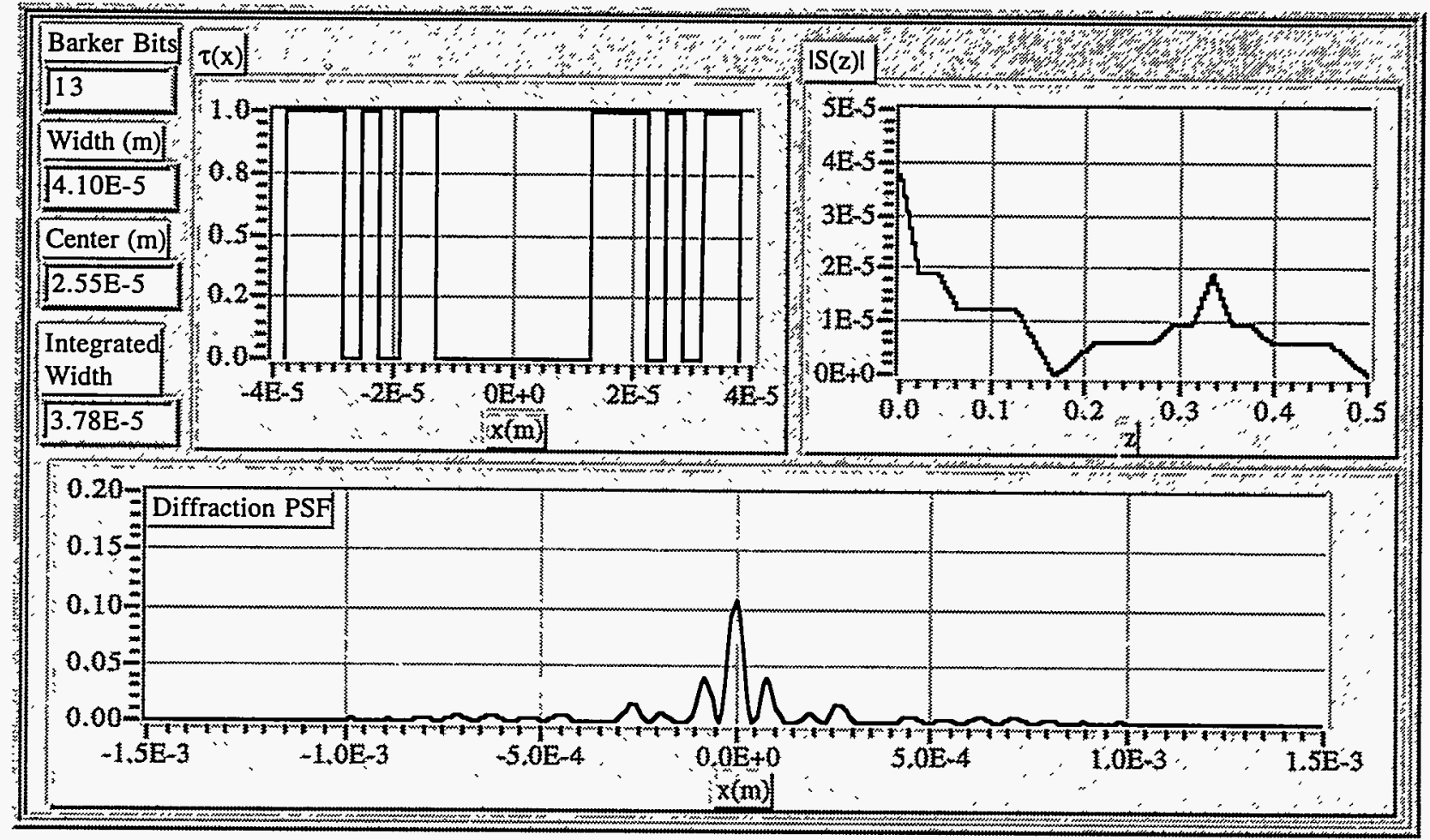

Figure A8: 13 Bit Barker Code Results 


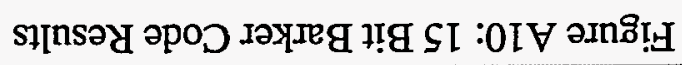



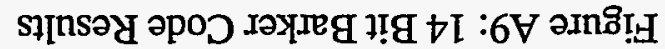

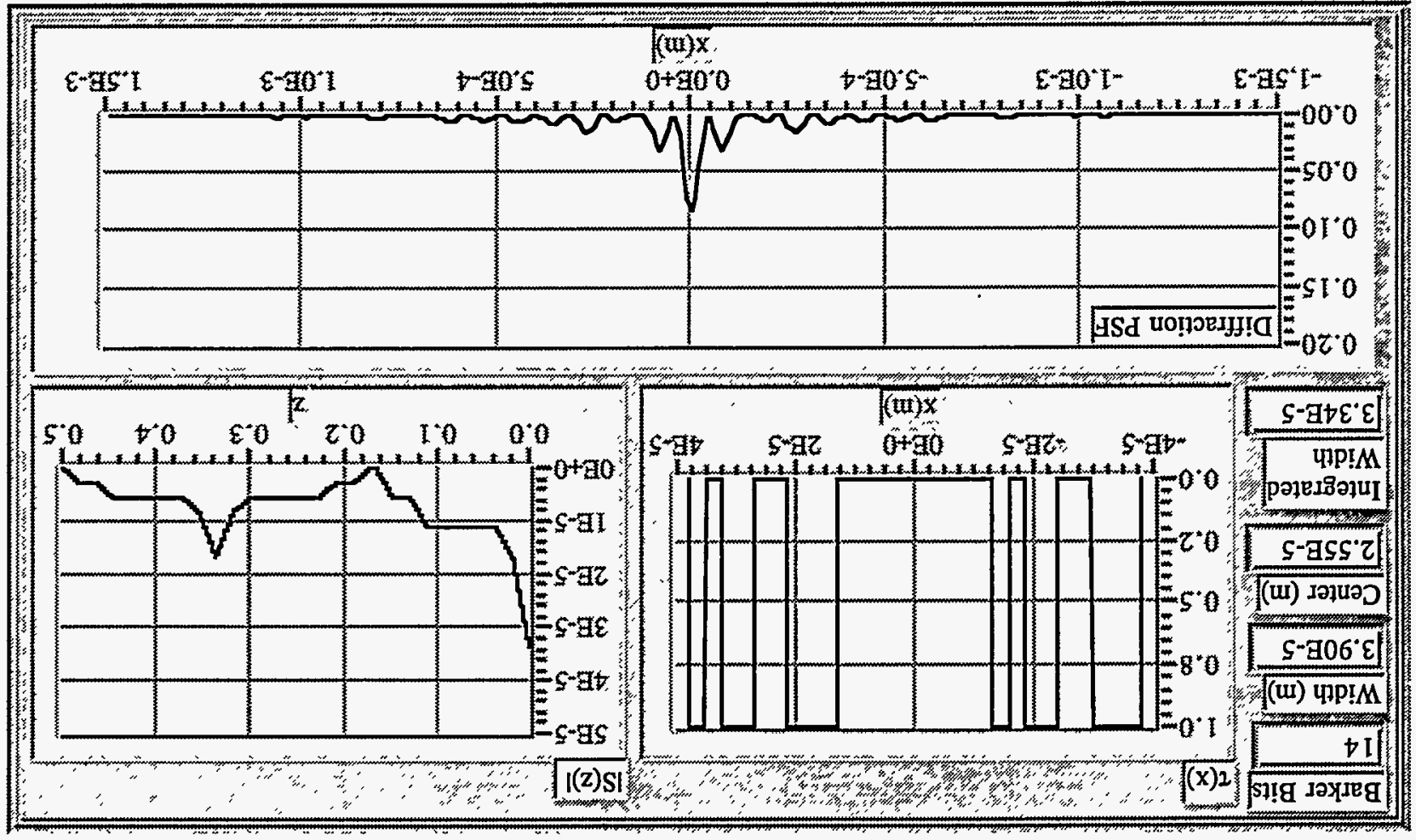




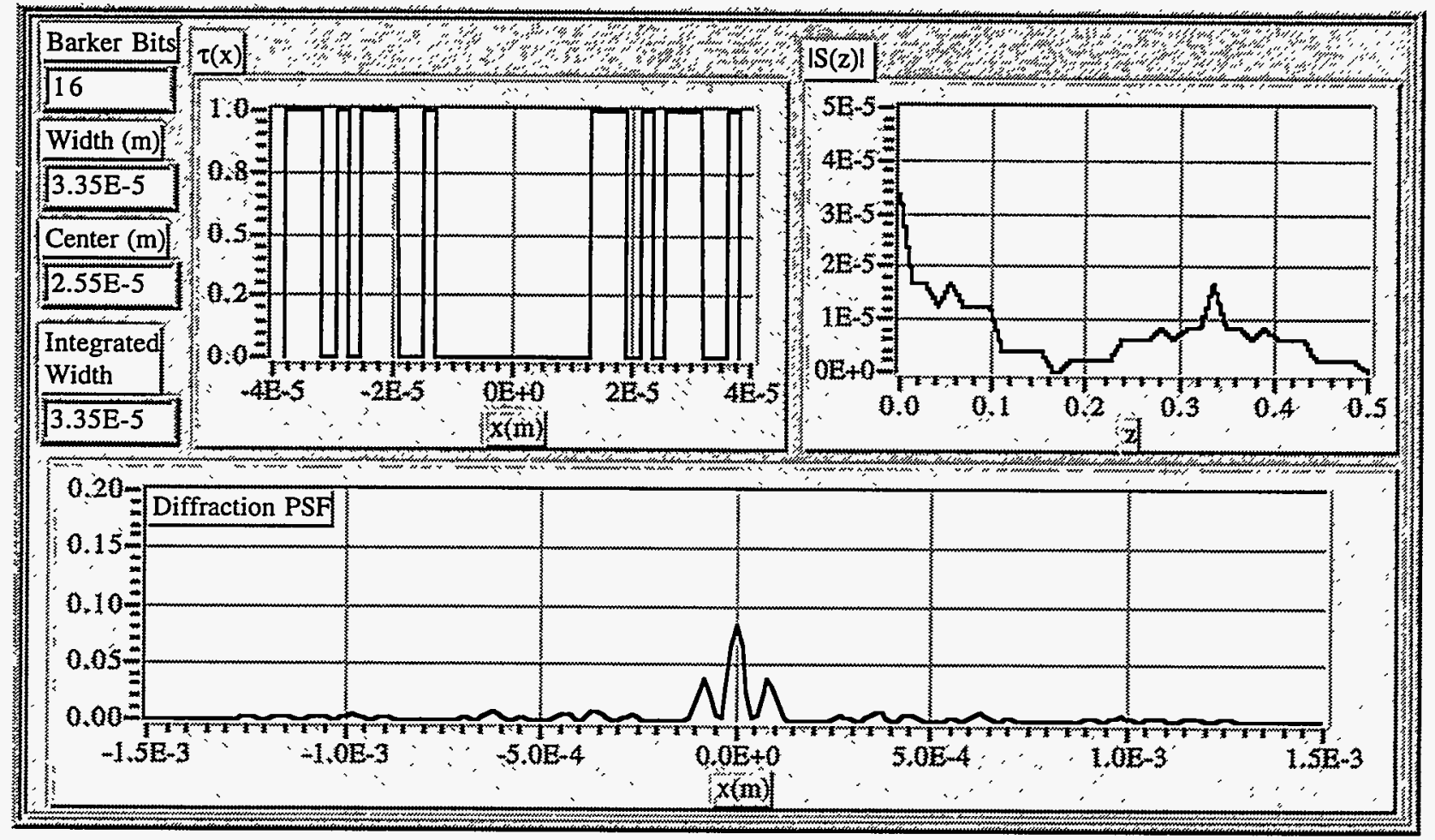

Figure A11: 16 Bit Barker Code Results

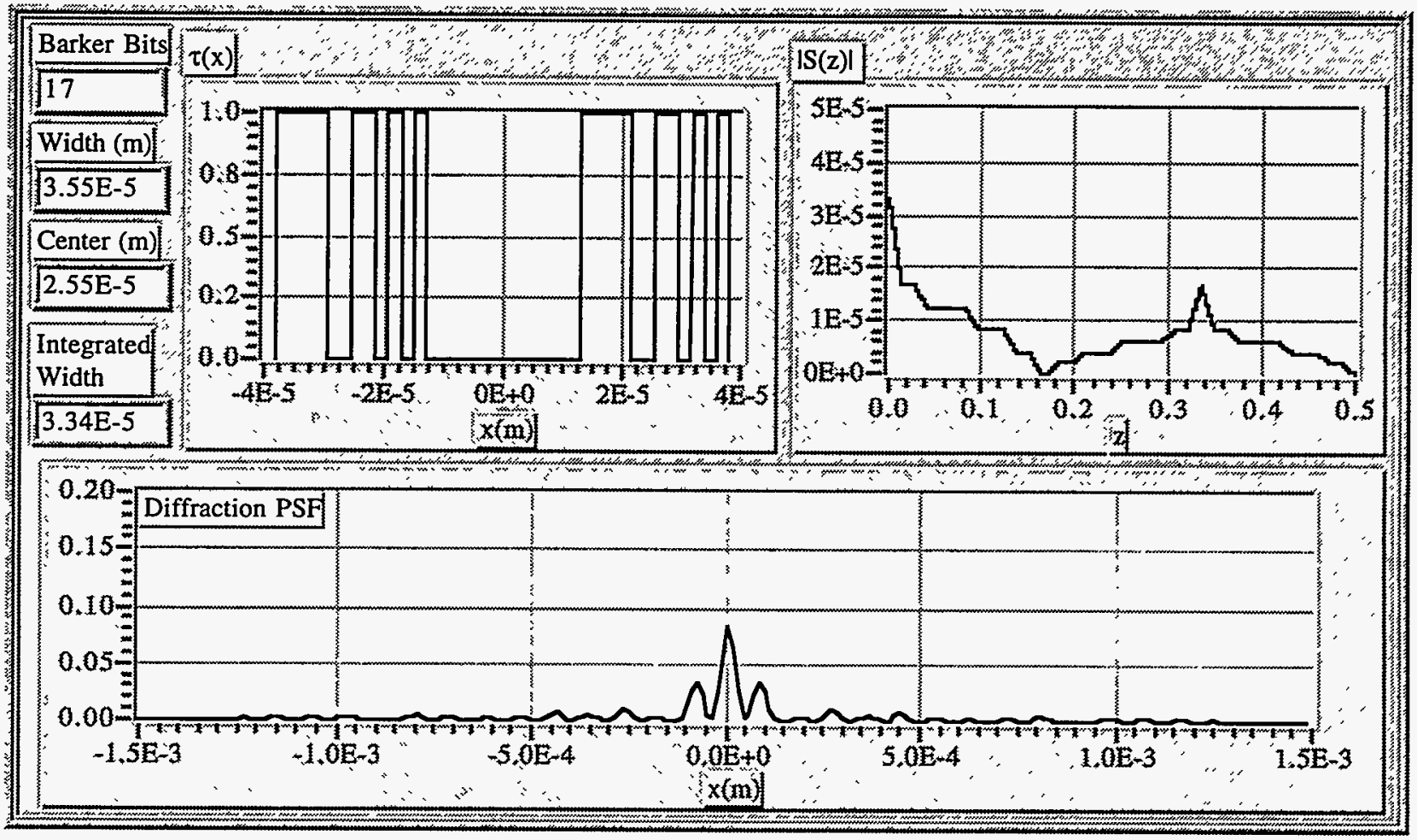

Figure A12: 17 Bit Barker Code Results 




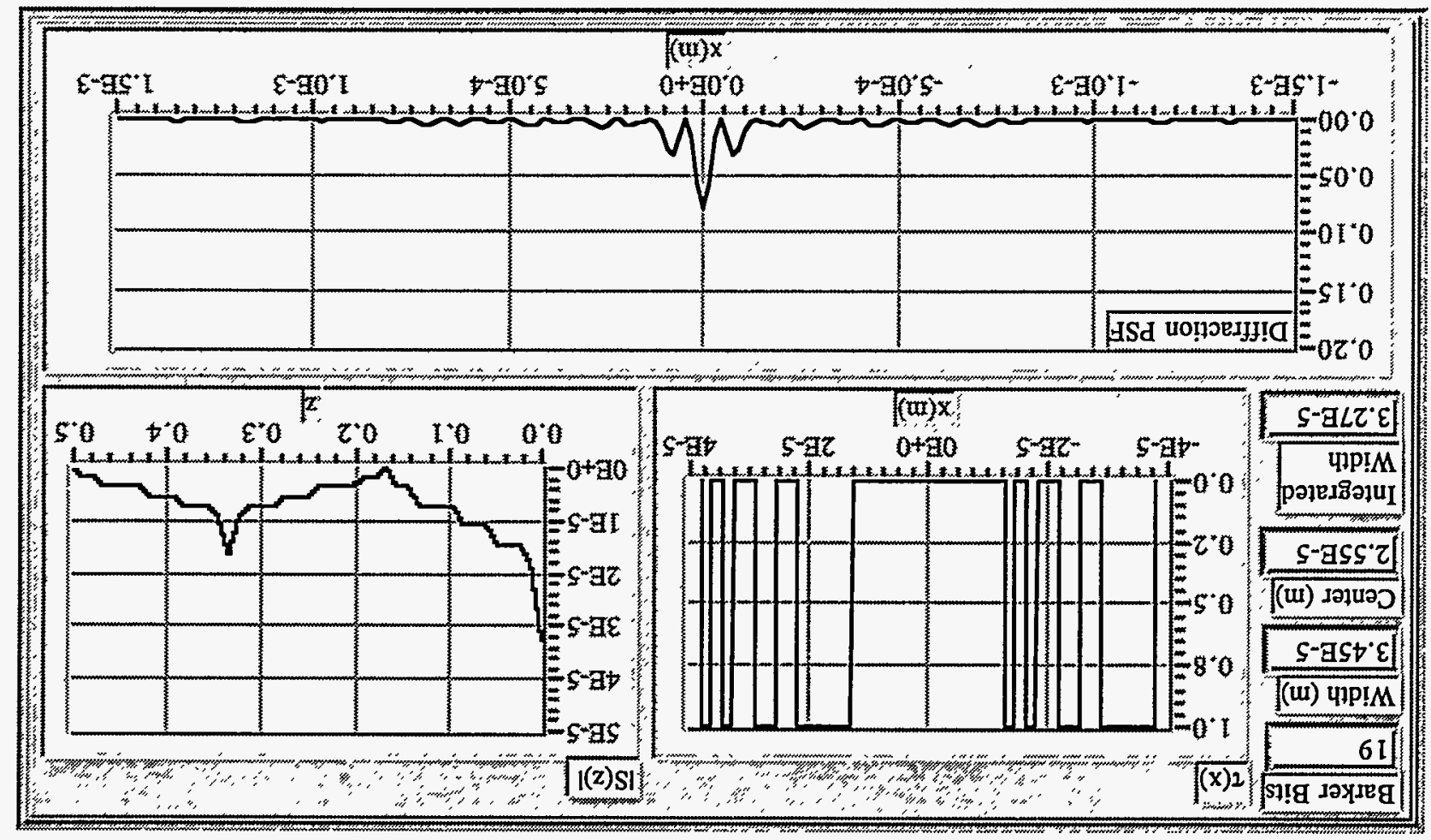

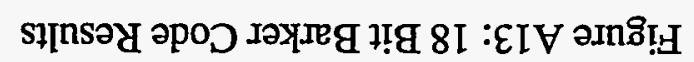






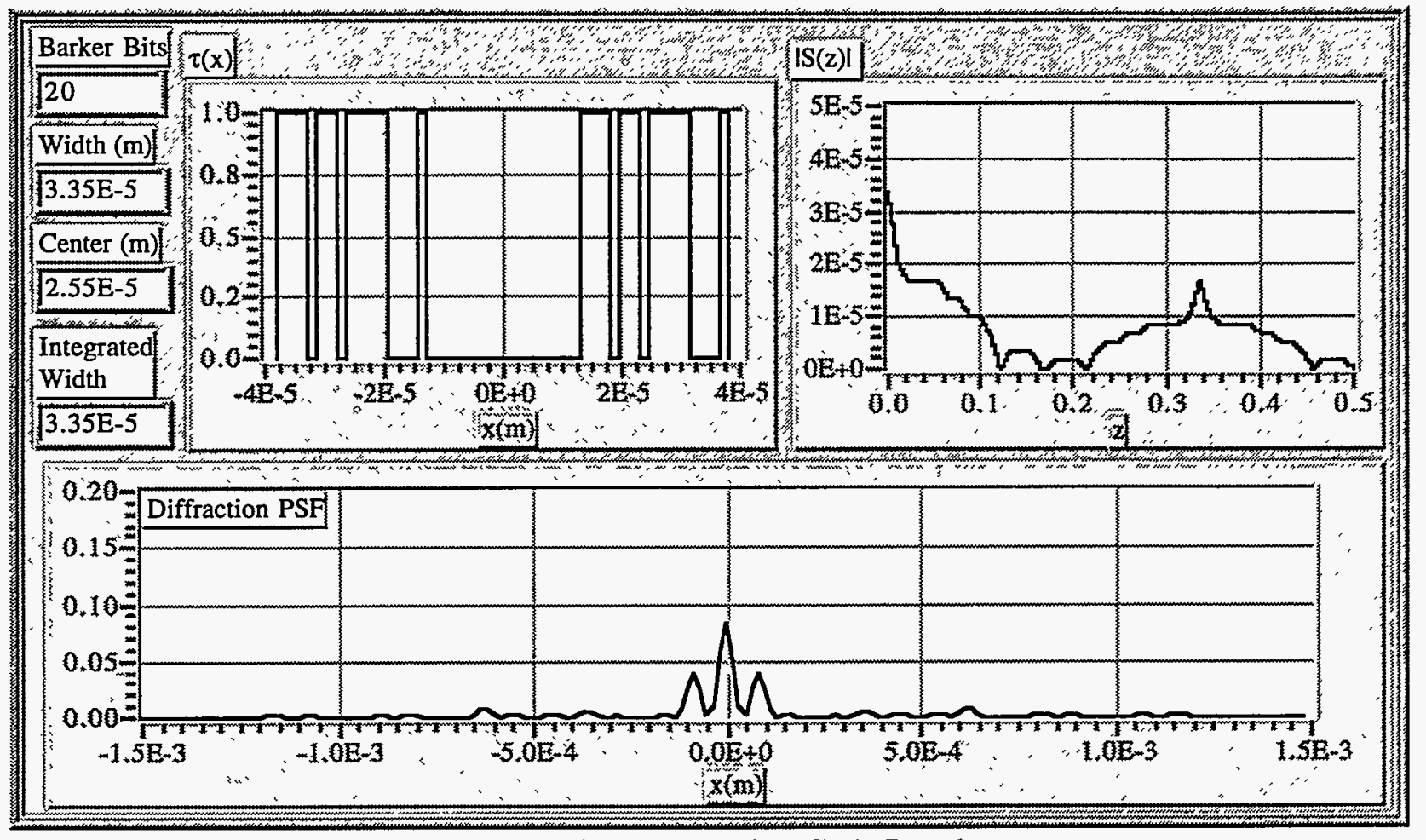

Figure A15: 20 Bit Barker Code Results

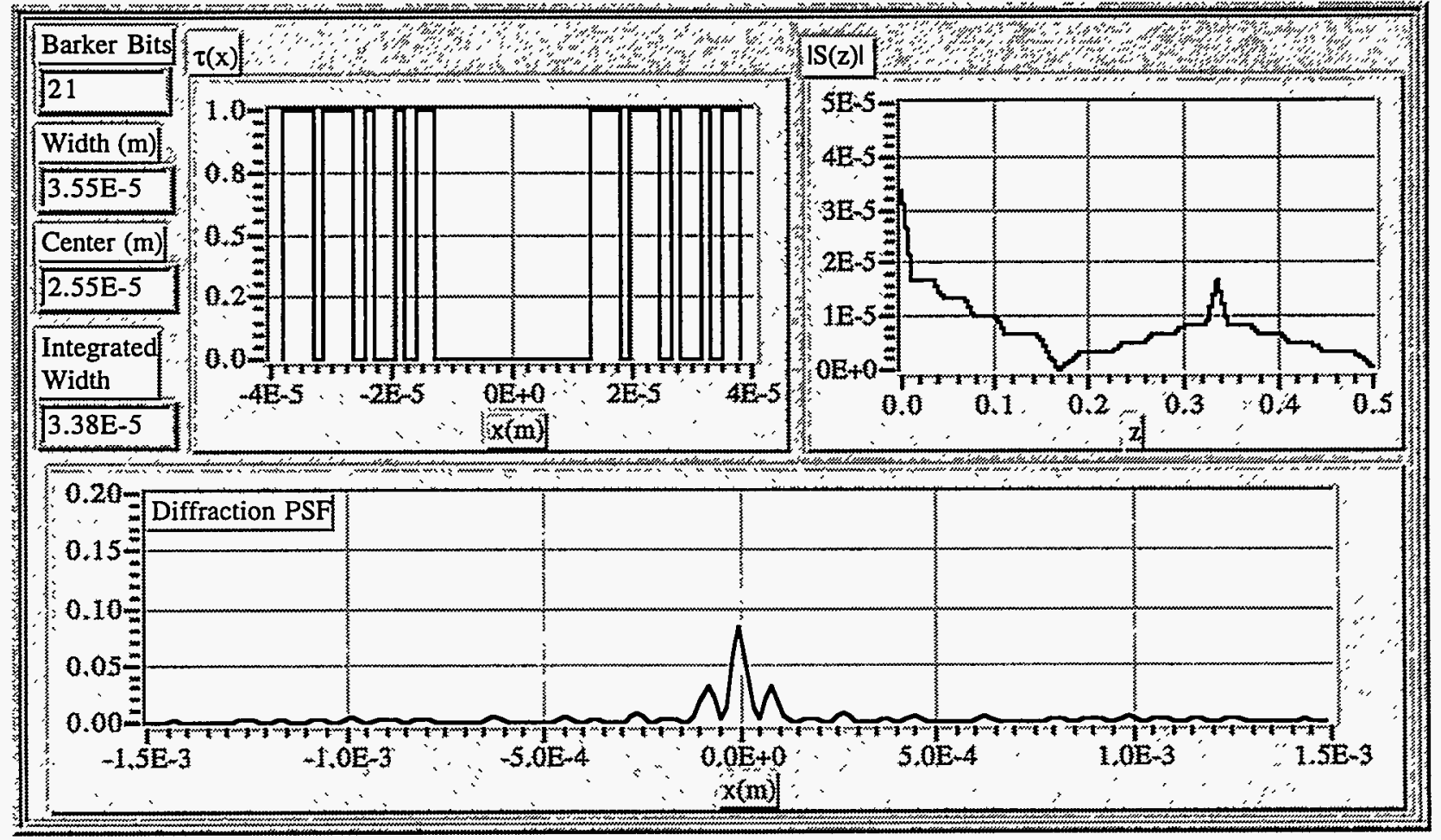

Figure A16: 21 Bit Barker Code Results 


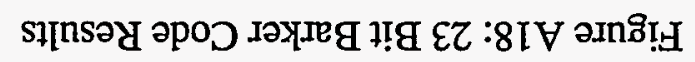

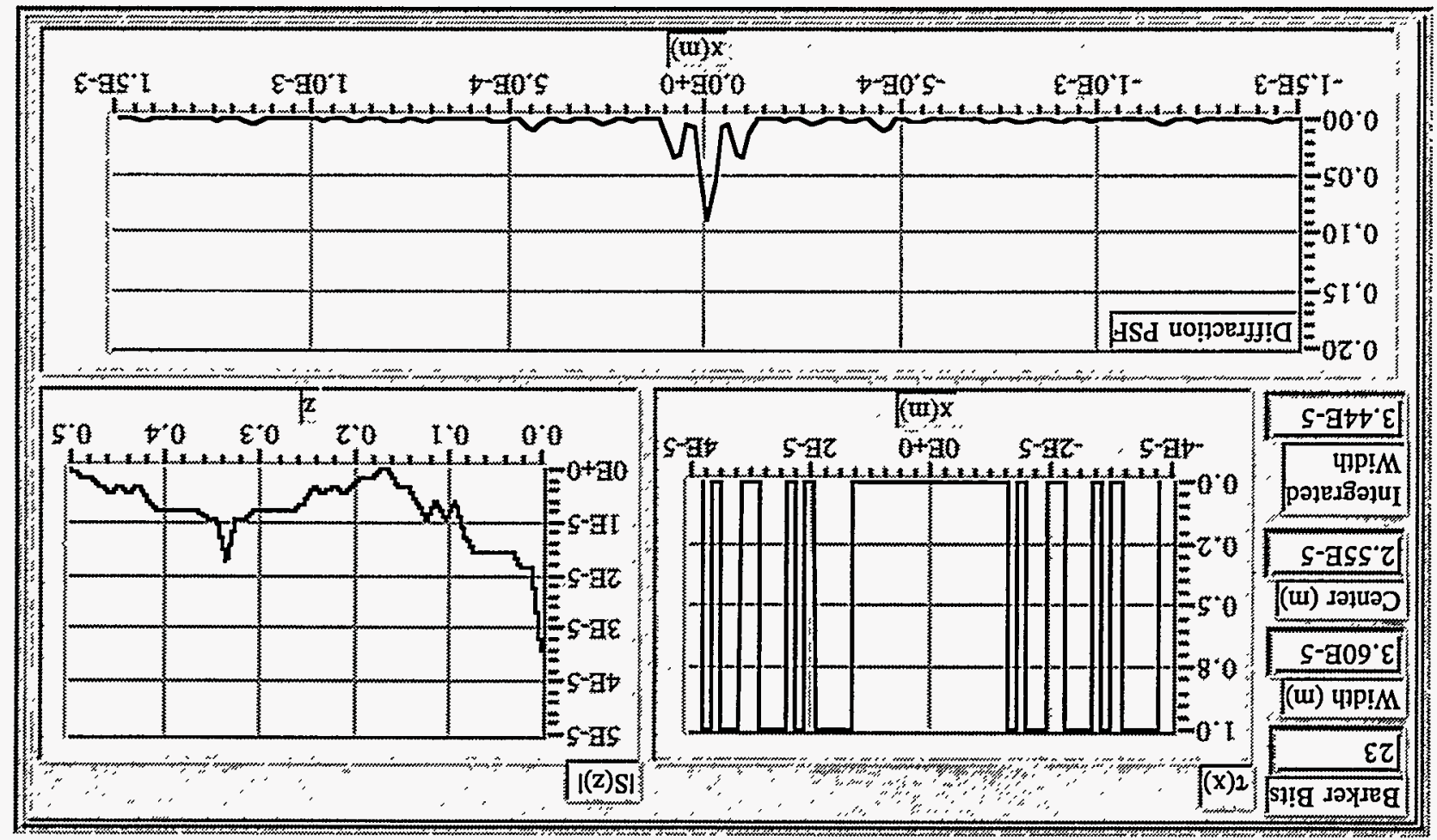

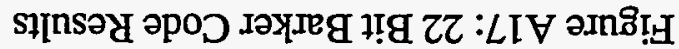

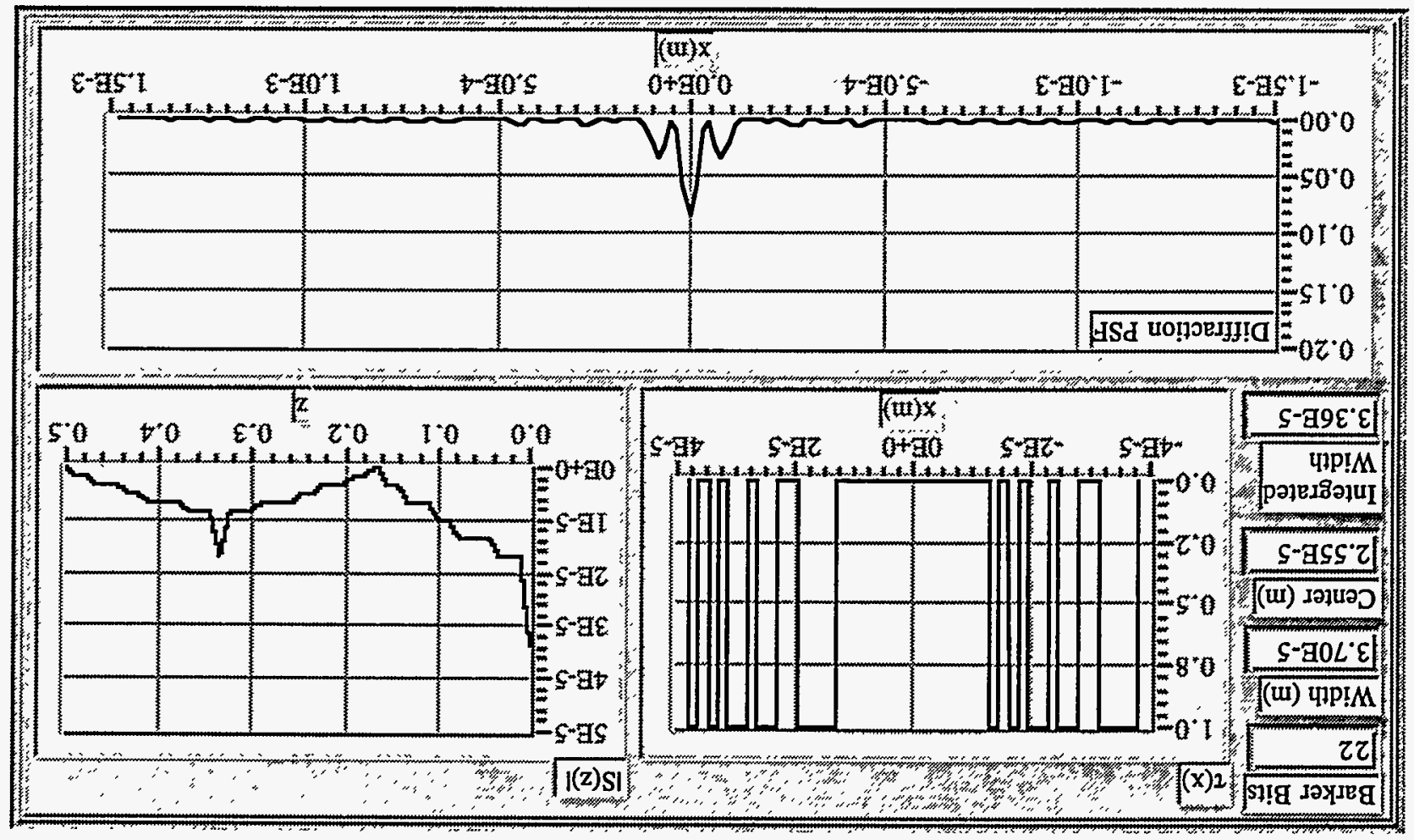




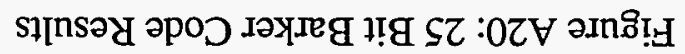



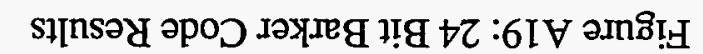








Figure A21: 26 Bit Barker Code Results



Figure A22: 27 Bit Barker Code Results 




Figure A23: 28 Bit Barker Code Results

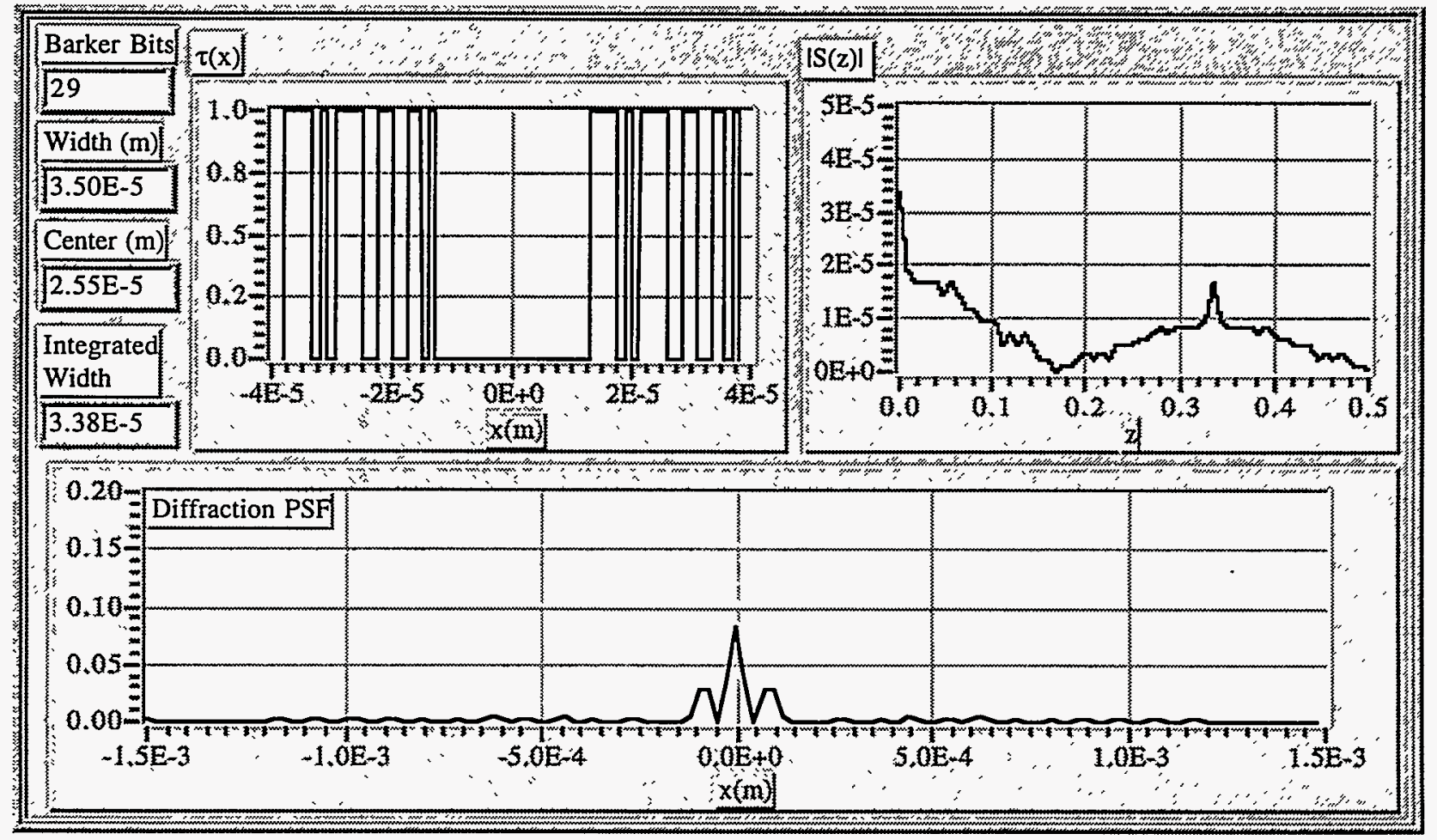

Figure A24: 29 Bit Barker Code Results 


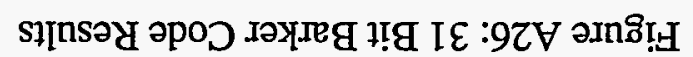

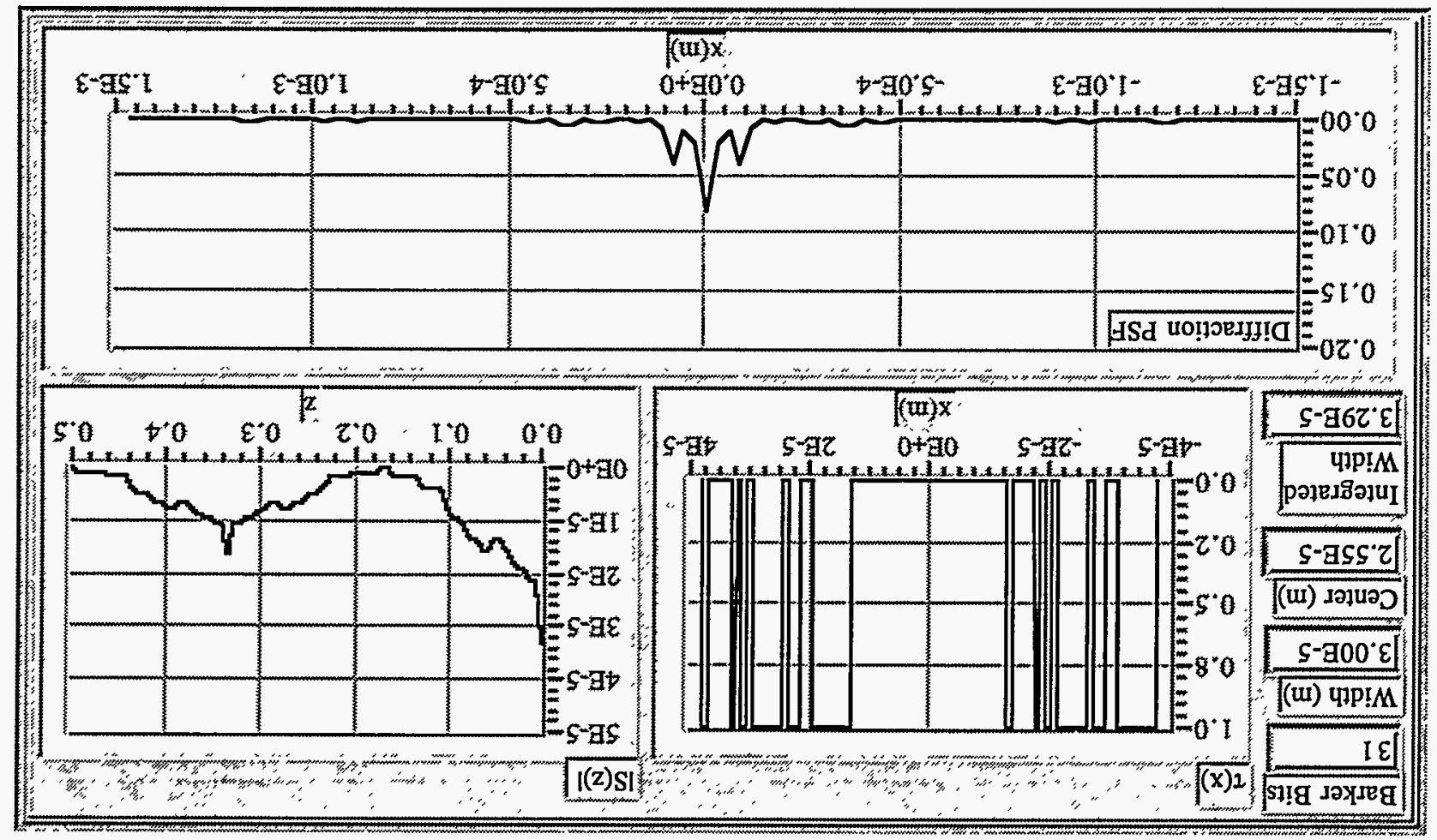

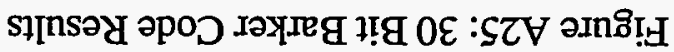

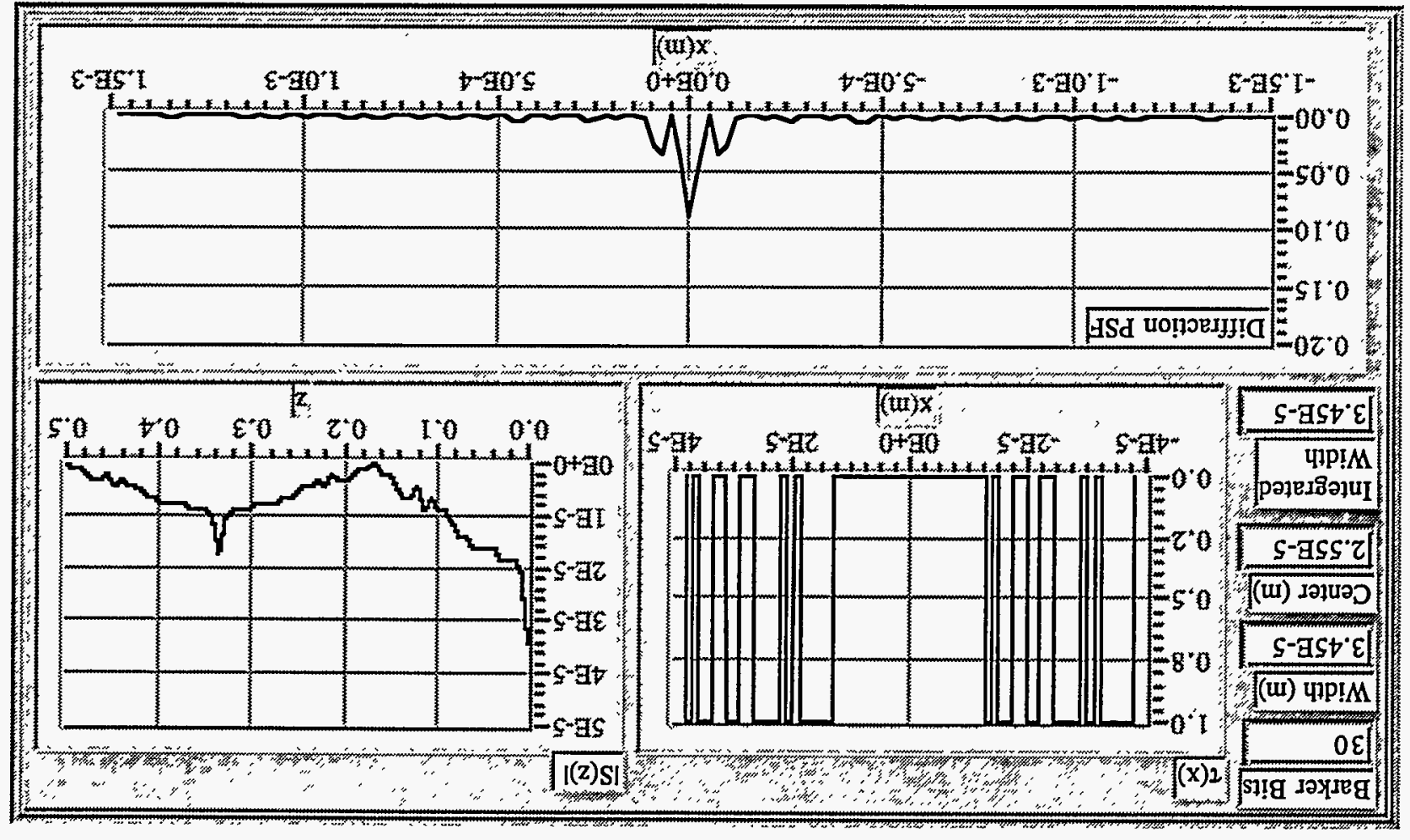




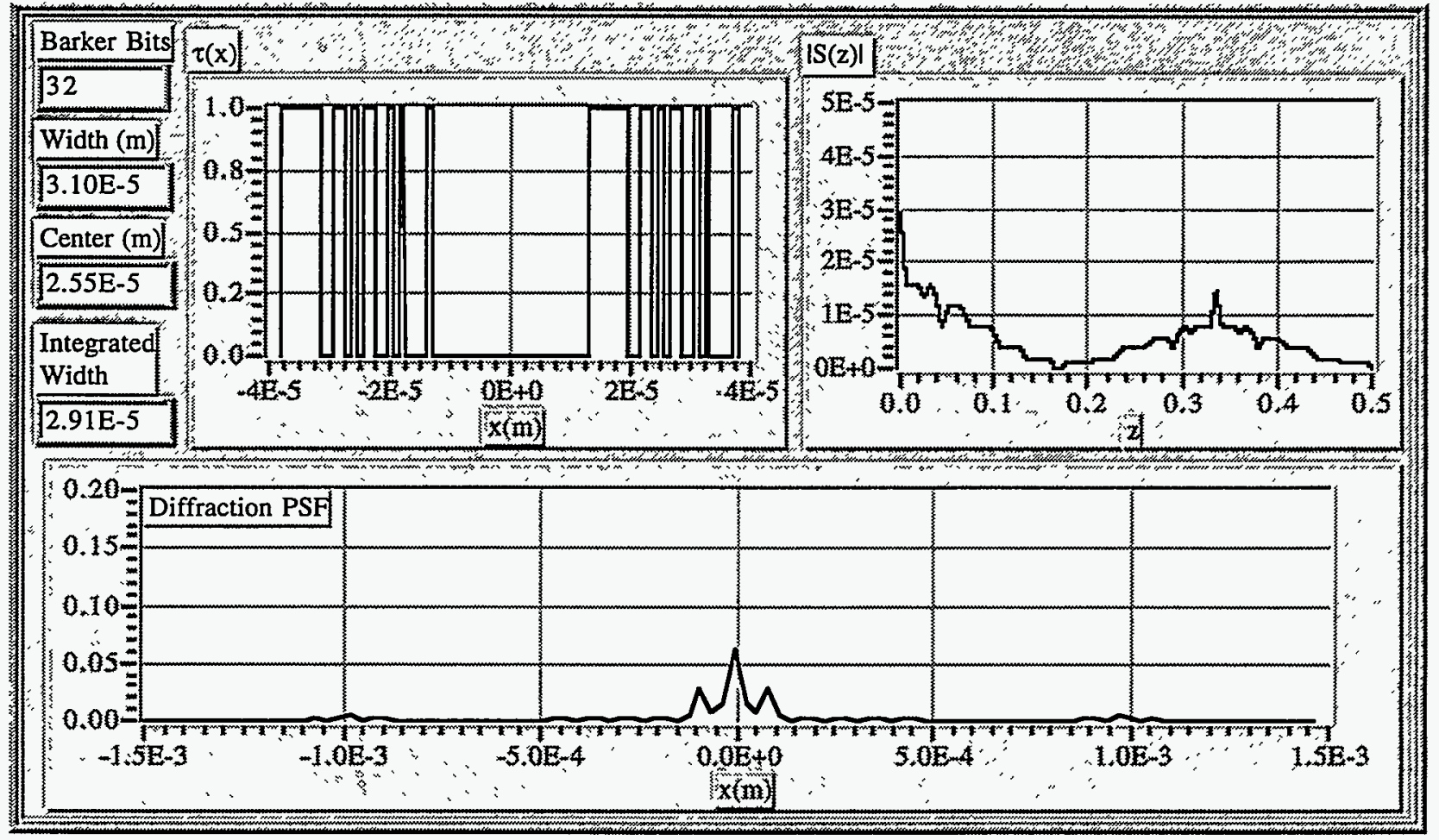

Figure A27: 32 Bit Barker Code Results

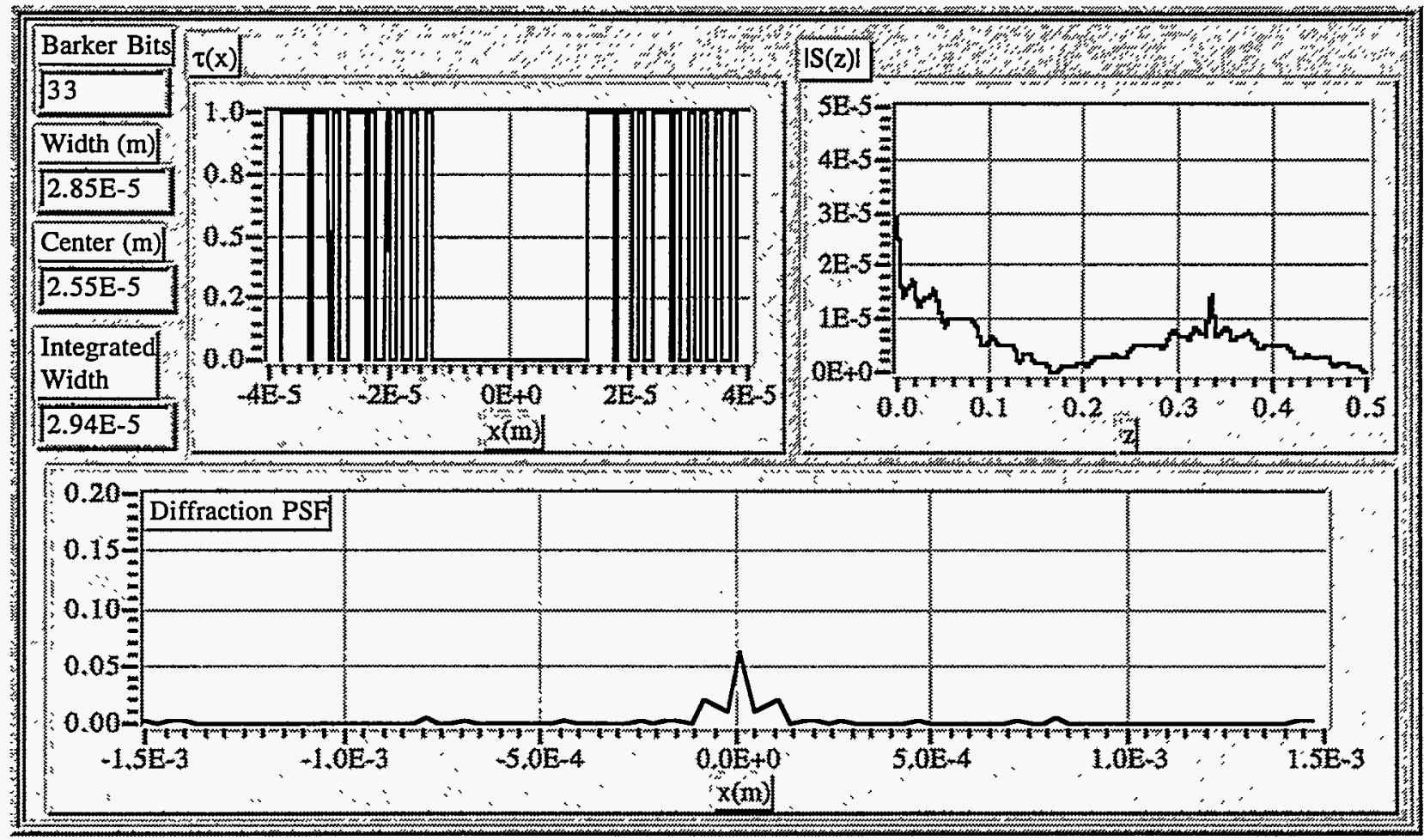

Figure A28: 33 Bit Barker Code Results 


\section{DISTRIBUTION:}

10980 S. M. Gentry, 9225

10980 C. A. Boye, 9225

10972 A. J. Medina, 9222

10972 D. A. Reynolds, 9205

$10980 \quad$ R. E. Abbink, 9225

10980 W. C. Sweatt, 9225

10980 T. D. Henson, 9225

10972 J. M. Falls, 9222

10972 J. L. Guillen, 9222

10972 T. G. Bates, 9222

10972 R. J. Blake, 9222

10972 R. R. Kay, 9222

10966 K. J. Jefferson, 9217

10965 K. R. Lanes, 9211

10965 G. W. Treaver, 9211

10365 O. M. Solomon, 1742

10952 M. S. Rogers, 2151

10328 L. S. Weichman, 2674

10320 LDRD Office, 1010

19018 Central Technical Files, 8523-2

70899 Technical Library, 13414

10619 Technical Publications, 13416

100100 Document Processing For DOE/OSTI, 7613-2 